NISTIR 8365

\title{
A Collaborative Robot Work-Cell Testbed for Industrial Wireless Communications
}

\author{
Yongkang Liu \\ Mohamed Hany \\ Karl Montgomery \\ Richard Candell
}

This publication is available free of charge from:

https://doi.org/10.6028/NIST.IR.8365

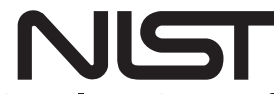

National Institute of Standards and Technology

U.S. Department of Commerce 
NISTIR 8365

\title{
A Collaborative Robot Work-Cell Testbed for Industrial Wireless Communications
}

\author{
Yongkang Liu \\ Mohamed Hany \\ Karl Montgomery \\ Richard Candell \\ Intelligent Systems Division \\ Engineering Laboratory
}

This publication is available free of charge from:

https://doi.org/10.6028/NIST.IR.8365

May 2021

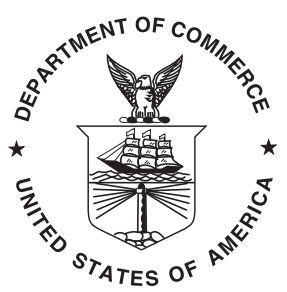

U.S. Department of Commerce Gina M. Raimondo, Secretary

National Institute of Standards and Technology James K. Olthoff, Performing the Non-Exclusive Functions and Duties of the Under Secretary of Commerce for Standards and Technology \& Director, National Institute of Standards and Technology 
Certain commercial entities, equipment, or materials may be identified in this document in order to describe an experimental procedure or concept adequately. Such identification is not intended to imply recommendation or endorsement by the National Institute of Standards and Technology, nor is it intended to imply that the entities, materials, or equipment are necessarily the best available for the purpose.

National Institute of Standards and Technology Interagency or Internal Report 8365

Natl. Inst. Stand. Technol. Interag. Intern. Rep. 8365, 101 pages (May 2021)

This publication is available free of charge from: https://doi.org/10.6028/NIST.IR.8365 


\begin{abstract}
A new testbed design for assessing the performance of wireless techniques in support of industrial operations is proposed. For this, a collaborative robot work-cell manufacturing scenario, which serves as the emulated cyber-physical system model in the testbed was developed. Specifically, the information exchanged between networked work-cell components, e.g., programmable logic controllers, robotic arms, and machining tools, was carried out via messages of industrial communication protocols; network connections employed were both Ethernet-based wired links and alternative wireless interfaces. A measurement framework was introduced to collect various system events from both work-cell operations and network traffic; a distributed clock synchronization scheme was developed to ensure the temporal consistency of measurement records at distributed sources. To facilitate offline performance analysis and data exploration, a new data pipeline was adopted that uses the graph database for testbed data management. The testbed has been in use in evaluating industrial wireless performance by the NIST team and industrial partners.
\end{abstract}

\title{
Keywords
}

Cyber-physical system (CPS); smart manufacturing; testbed; wireless communications. 


\section{Table of Contents}

1 Introduction 1

2 Related Work 2

3 Overview of the Testbed Architecture 4

4 Production Modules 5

4.1 Work-Cell Workflow 6

$\begin{array}{lll}4.2 & \text { Supervisor } & 7\end{array}$

4.2.1 Scheduler 8

4.2.2 HMI 9

$\begin{array}{lll}4.2 .3 & \text { Job Buffers } & 10\end{array}$

4.2.4 Production Tasks 10

$\begin{array}{lll}4.3 & \text { Tooling Machines } & 12\end{array}$

$\begin{array}{lll}4.4 & \text { Robots } & 15\end{array}$

4.5 Coordination between Work-Cell Modules 16

4.5.1 Coordination in Initialization 18

4.5.2 Coordination in Job Operations 20

5 Network Components $\quad 26$

5.1 Communications in Work-Cell Applications 27

5.1.1 Remote HMI Operations 27

5.1.2 Supervisor-CNC Machines Interactions 28

5.1.3 Supervisor-Robots Interactions 29

$\begin{array}{ll}\text { 5.1.4 Robotic Subsystem Communications } & 29\end{array}$

5.1.5 A Summary of Communication Traffic 30

5.2 Networking Architecture 30

5.3 Wireless Extension 32

6 Measurement Framework 35

6.1 Measurement Data Collection 36

6.1.1 Operational Data Collection 36

6.1.2 Network Traffic Captures 37

6.2 Time Synchronization 39

6.2.1 Precision Time Protocol 40

6.2.2 Timestamp Formats 41

7 Data Management $\quad \mathbf{4 2}$

$\begin{array}{lll}7.1 & \text { Graph Database } & 42\end{array}$

7.1.1 Graph Data Model 42

7.1.2 Building Blocks 43

$\begin{array}{lll}7.2 & \text { Data Importing Pipeline } & 47\end{array}$

7.2.1 Data Preprocessing 48

7.2.2 Feature Extraction 48

7.2.3 Graph Insertion 48 
7.2.4 Graph Tuning 49

8 Data Analysis \& Graph Exploration 49

8.1 Graph Database Schema 49

8.2 Experiment Configurations 51

8.3 Preliminary Results $\quad 52$

9 Conclusion $\quad 55$

References $\quad 56$

Appendices $\quad 60$

A Communication Messages in the Testbed 60

A.1 Basic Packet Format 60

$\begin{array}{lll}\text { A.2 } & \text { ADS/AMS } & 62\end{array}$

A.3 Modbus 65

B Beckhoff PLC Development $\quad 68$

B.1 PLC Data Collection $\quad 68$

$\begin{array}{lll}\text { B.1.1 Start a PLC Measurement } & 68\end{array}$

B.1.2 Adding New PLC Variables in the Subscription List 70

B.2 PLC Time Synchronization $\quad 72$

C Translating Testbed Data to Graph Database 75

C.1 Graph Nodes, Relationships, and Their Properties 75

$\begin{array}{lll}\text { C.1.1 Nodes } & 75\end{array}$

$\begin{array}{ll}\text { C.1.2 Relationships } & 80\end{array}$

C.2 Data Importing Flow 81

$\begin{array}{lll}\text { C.2.1 Overview } & 81\end{array}$

C.2.2 Packet Dissection 83

C.2.3 Coupling Packet Captures in Communication Links 83

C.2.4 Cypher Queries and Python-Neo4j Interface 85

$\begin{array}{lll}\text { D Testbed Equipment Specifications } & 87\end{array}$

E Network Diagrams in Testbed Experiments $\quad 88$

$\begin{array}{lll}\text { F Acronyms } & 91\end{array}$

\section{List of Tables}

Table 1 Feature comparison with existing industrial wireless evaluation platforms 3

Table 2 Specifications of sampled data flows between work-cell components 32

Table 3 Measurement data timestamp settings via PTP 40

Table 4 ADS commands $\quad 63$

Table 5 Nodes and their property tags in the graph for static objects 75

Table 6 (:Message) nodes and their property tags in the graph 77

Table 7 (:Transaction) nodes and their property tags in the graph 78 
Table 8 Physical action nodes and their property tags in the graph 79

Table 9 QoSReport nodes and their property tags in the graph 80

Table 10 Relationships and their creation conditions in the graph 81

Table 11 Testbed hardware and software specifications $\quad 87$

\section{List of Figures}

Fig. 1 The NIST industrial wireless testbed architecture 4

Fig. 2 Product modules in the testbed 5

Fig. 3 Illustration of work-cell interactions in a tooling path 6

Fig. 4 Function modules in the supervisor PLC. 7

Fig. 5 Work-cell HMI and the control functions 9

Fig. 6 State machines of orders and queues 11

Fig. 7 The online order editor module in HMI $\quad 12$

Fig. 8 Architecture of the CNC machine emulator 13

Fig. 9 CNC state machine $\quad 14$

Fig. 10 A UR3 collaborative robot system 15

Fig. 11 The state machine of the UR3 robot in the work-cell 16

Fig. 12 The flow diagram in a UR3 robot cycle 17

Fig. 13 Timeline of initialization steps. 19

Fig. 14 Reset coordination between work-cell modules 20

Fig. 15 Coordination in a tooling procedure $\quad 20$

Fig. 16 Module interactions in a part transition case $\quad 22$

Fig. 17 Module interactions in a part inspection case 25

Fig. 18 Communications for remote HMI operations 27

Fig. 19 The supervisor-CNC Communications 28

Fig. 20 The supervisor-robot communications 30

Fig. 21 Communication between the robot controller and the F/T sensor 31

Fig. 22 Work-cell network architecture using full wired Ethernet connections 33

Fig. 23 Wireless extension to the work-cell network architecture 33

Fig. 24 Illustration of Layer 2 forwarding through the Ethernet-WLAN adapter 34

Fig. 25 The testbed measurement framework $\quad 35$

Fig. 26 Network traffic test access point (TAP) device 38

Fig. 27 Network traffic probes in the testbed network 38

Fig. 28 Packet header samples from TAP and wireless sniffer captures 39

Fig. 29 PTP time synchronization using LinuxPTP 41

Fig. 30 Data processing flow from factory work-cell to database 43

Fig. 31 The single-run testbed data model in the graph database 44

Fig. 32 Realized schema of the graph database 50

Fig. 33 Wired baseline physical action time $\quad 52$

Fig. 34 Wireless baseline physical action time $\quad 53$

Fig. 35 Wireless with 2500 pps traffic physical action time (run 1) 53 
Fig. 36 Wireless with 2500 pps traffic physical action time (run 2)

Fig. 37 Wireless with 2x1250 packets/s traffic physical action time 54

Fig. 38 Histograms of Transaction Latency for Various Experimental Scenarios 54

Fig. 39 Histograms of Physical Action Time for Various Experimental Scenarios 55

Fig. 40 Construction of a TCP/IP-Ethernet data packet $\quad 60$

Fig. 41 TCP header $\quad 61$

Fig. 42 IP header $\quad 61$

Fig. 43 UDP header $\quad 62$

Fig. 44 ADS packet frame 63

Fig. 45 Handshakes of ADS message transactions in a WireShark trace file 64

Fig. 46 Construction of a Modbus TCP data packet $\quad 65$

Fig. 47 Modbus register address $\quad 66$

Fig. 48 Modbus function codes $\quad 66$

Fig. 49 Modbus function of reading holding registers 66

Fig. 50 Modbus function of presetting single register 67

Fig. 51 PLC measurement data in CSV 68

Fig. 52 PLC measurement configuration in XML $\quad 69$

Fig. 53 Load PLC measurement configuration in HMI 69

Fig. 54 The design of PLC measurement 71

Fig. 55 Time synchronization between PLC and time server through PTPv2 72

Fig. 56 PTP end-to-end delay mechanism 73

Fig. 57 Distributed clock adjustment in PTP time synchronization 73

Fig. 58 Implementing the pipeline of importing testbed data into GDB 82

Fig. 59 Multiple network captures in a control command transaction $\quad 84$

Fig. 60 Graph nodes in a control command transaction $\quad 84$

Fig. 61 Graph nodes and the established relationships in a control transaction 85

Fig. 62 Coupling procedures to pair network captures in a communication transaction. 86

Fig. 63 Testbed network diagram with only work-cell connections $\quad 88$

Fig. 64 Network diagram with full wired connections and measurement data links $\quad 89$

Fig. 65 Testbed network diagram with wireless connections 90 


\section{Introduction}

Wireless applications are among key enabling technologies in smart manufacturing innovations that promise flexible deployment capability and mobile support for data transmissions on the factory floor [1]. Emerging wireless techniques, such as recent wireless local area networks (WLAN), also known as Wi-Fi 6, and the fifth-generation (5G) cellular networks, are anticipating to land their killer applications in industrial communication scenarios for which they are planning technical deployment routes. As industrial plants are complex cyber-physical systems (CPS), general wireless communication metrics cannot fully meet the need of comprehensively evaluating wireless impact onto manufacturing activities which are closely related to production efficiency and operation safety. Among the first attempts, standard development organizations (SDO) have proposed multiple classification methods of identifying different industrial communication use cases and their specific performance requirements, e.g., latency and reliability metrics [2]. Measurement sciences that assess wireless techniques in support of manufacturing practices are essential to the success of industrial wireless communications [3].

The National Institute of Standards and Technology (NIST) has been sponsoring industrial wireless performance studies in its long-term research program entitled "Trustworthy Systems, Components, and Data for Smart Manufacturing" [4]. As a key supporting unit in this program, the industrial wireless system (IWS) lab at NIST anticipates low-latency and high-reliability wireless communications would play an important role in manufacturing areas. Wireless links can support real-time process monitoring and automation control, which involve various industrial devices, such as programmable logic controllers (PLCs), field sensors and actuators, and machine tools. In addition, it is anticipated that there will be a mixed network topology with wired and wireless connections that carry standardized and proprietary industrial communication messages. These new features raise challenging issues in the design and implementation of industrial wireless communications. However, limited system models and testing platforms are readily available for engineers to evaluate emerging industrial wireless solutions in real environments.

This report presents our recent measurement activities at NIST in collaboration with industrial partners. A testbed was designed by replicating various data flows in a managed production environment for the purpose of evaluating the impact of industrial communication systems on the factory automation processes. A collaborative robot work-cell was emulated as the CPS model whose service requirements are representative of a typical industrial application. The testbed characterizes deterministic and reliable communication needs between work-cell components in a machine tending application. Measurement methods were utilized to ensure the compliance and consistency of the observed data from distributed monitoring points regarding production states and network traffic. A time synchronization design was used to keep the collected data records aligned in time. Experiments were performed with the Ethernet-based wired configuration and the wireless alternative using WLAN devices. A data workflow based on the graph database (GDB) technique was developed to streamline the processing and analysis of heterogeneous testbed 
data in exploring CPS performance factors. Since the testbed design has been partly introduced in our earlier publications [5-7], this report aims to serve as a comprehensive review of the testbed architecture, component module details, related measurement activities, and lessons learned from these implementations. The report's goal is to assist industrial engineers, wireless designers, and CPS researchers when they are designing similar evaluation platforms and dealing with typical industrial wireless system models. Note that cybersecurity discussions of industrial wireless communications are not in the scope of this report. Interested readers can refer to the peer project in the NIST program [8].

The remainder of this report is organized as follows. Section 2 briefly discusses the related work of the testbed project. Section 3 provides an overview of the testbed architecture. Hereafter, design topics are elaborated in the following sections. Production-related modules including the Supervisor, computer numerical control (CNC), and robots are introduced in Section 4; the network design is addressed in Section 5 where both wired and wireless networking issues are discussed; the measurement framework is described in Section 6. The testbed data are stored and managed in a non-relational (NoSQL) database organized by a graph whose model and processing flow are defined in Section 7. We will explore the latency performance of the selected communication links in Section 8. The presented experimental configurations and preliminary analysis results are used to showcase the capability and potentials of this testbed. In the end, Section 9 will summarize the main takeaways and discuss the future work of the testbed.

\section{Related Work}

Trending efforts on developing evaluation methods and tools for emerging industrial wireless communications can be classified into three major areas: theoretical analysis, simulations, and testbed platforms. Based on field measurements of industrial radio channels and traffic traces, statistical models have been developed to characterize channel propagation features, interference variations, and usage patterns. For example, NIST has performed a series of channel measurement campaigns in different topographical sites to understand the industrial channel characteristics [9]. Considering the complexity of modern manufacturing processes, both network performance and manufacturing productivity need to be captured thoroughly, especially when transmissions are closely associated with process precision and plant safety. Therefore, performance evaluation is still mainly conducted through computer simulations and in testbed platforms with real hardware configurations.

The computer simulation approach has been widely used in studying the performance of networked systems, which can flexibly adjust the studied network scale while tuning configuration parameters in various regular/extreme test cases. Recently, the co-simulation of physical processes and associated network activities has gained the attentions of CPS researchers. For example, a co-simulator design, that combines the production pipeline and wireless network operations into an integrated discrete-time framework, was proposed in $[10,11]$. However, the trustworthiness of simulation results to estimate the real system performance largely relies on the model accuracy and the computational complexity which 
often point to the opposite directions given the simulator's limits. Therefore, it is critical to design a testbed platform for both validating theoretical models and demonstrating the performance of industrial wireless solutions in realistic operational and environmental configurations, which is a convincing approach to encourage the adoption of emerging techniques.

Table 1. Feature comparison with existing industrial wireless evaluation platforms (reprinted from [7])

\begin{tabular}{|c|c|c|c|c|c|c|c|c|c|}
\hline & \multirow{2}{*}{$\begin{array}{l}\text { Application } \\
\text { Domain }\end{array}$} & \multirow{2}{*}{ Use Case } & \multicolumn{3}{|c|}{ System Setup } & \multicolumn{2}{|c|}{ Data Collection } & \multicolumn{2}{|c|}{ Evaluation } \\
\hline & & & $\begin{array}{l}\text { Physical } \\
\text { System }\end{array}$ & $\begin{array}{l}\text { Wireless } \\
\text { Network }\end{array}$ & RF Factor & Physical & Network & $\begin{array}{c}\text { Data } \\
\text { Process }\end{array}$ & $\begin{array}{c}\text { CPS } \\
\text { Metrics }\end{array}$ \\
\hline $\begin{array}{l}\text { This } \\
\text { work }\end{array}$ & $\begin{array}{l}\text { Factory } \\
\text { Automation }\end{array}$ & $\begin{array}{l}\text { Robotic } \\
\text { Work-cell }\end{array}$ & $\begin{array}{l}\text { HW (PLC, } \\
\text { robots, } 10- \\
125 \mathrm{~Hz} \text { updates) }\end{array}$ & $\begin{array}{l}\text { HW (WLAN } \\
\text { IEEE } 802.11 \mathrm{~b} / \mathrm{g} / \mathrm{n} \\
\text { as presented) }\end{array}$ & $\begin{array}{l}\text { Indoor (Lab), } \\
\text { managed WLAN } \\
\text { interference } \\
\text { radios in } 2.4 \mathrm{GHz}\end{array}$ & $\begin{array}{l}\text { Device } \\
\text { logging, } \\
\text { remote } \\
\text { access }\end{array}$ & $\begin{array}{l}\text { Ethernet } \\
\text { /WLAN } \\
\text { sniffer }\end{array}$ & $\begin{array}{l}\text { Pipeline, } \\
\text { scripts, } \\
\text { graph } \\
\text { database }\end{array}$ & $\begin{array}{l}\text { Control } \\
\text { delay, } \\
\text { system } \\
\text { failure }\end{array}$ \\
\hline $\begin{array}{l}\text { Aminian } \\
2013[12]\end{array}$ & $\begin{array}{l}\text { Process } \\
\text { Automation }\end{array}$ & $\begin{array}{l}\text { Dual-Tank } \\
\text { level control } \\
\text { as presented }\end{array}$ & $\begin{array}{l}\text { SW (by } \\
\text { Simulink) }\end{array}$ & $\begin{array}{l}\text { SW (Wireless } \\
\text { Mesh with } \\
\text { IEEE } 802.15 .4 \text { ), } \\
\text { HIL (tentative) }\end{array}$ & SW & $\begin{array}{l}\text { Simulator } \\
\text { logging }\end{array}$ & $\begin{array}{l}\text { Simulator } \\
\text { logging }\end{array}$ & $\begin{array}{l}\text { Visual } \\
\text { inspection, } \\
\text { scripts }\end{array}$ & $\begin{array}{l}\text { Control, } \\
\text { I/O }\end{array}$ \\
\hline $\begin{array}{l}\text { Jecan } \\
2018[13]\end{array}$ & $\begin{array}{l}\text { Process } \\
\text { Automation }\end{array}$ & $\begin{array}{l}\text { Industrial } \\
\text { Wireless } \\
\text { Network } \\
\end{array}$ & No & $\begin{array}{l}\text { HW (WirelessHART } \\
\text { plus ISA100.11a) }\end{array}$ & Indoor (Lab) & No & $\begin{array}{l}\text { Network } \\
\text { manager }\end{array}$ & scripts & No \\
\hline $\begin{array}{l}\text { Ding } \\
2015 \text { [14] }\end{array}$ & $\begin{array}{l}\text { Process } \\
\text { Automation }\end{array}$ & $\begin{array}{l}\text { Wireless } \\
\text { Sensor \& } \\
\text { Actuation }\end{array}$ & $\begin{array}{l}\text { HW (valve } \\
\text { control, } 1 \mathrm{~Hz} \\
\text { updates) }\end{array}$ & $\begin{array}{l}\text { HW (WirelessHART, } \\
\text { ISA100.11a) }\end{array}$ & $\begin{array}{l}\text { Indoor (factory), } \\
\text { managed Zigbee } \\
\text { and WLAN in } \\
2.4 \mathrm{GHz}\end{array}$ & No & $\begin{array}{l}\text { Packet } \\
\text { sniffer }\end{array}$ & scripts & No \\
\hline $\begin{array}{l}\text { Liu, Q } \\
2018[15]\end{array}$ & $\begin{array}{l}\text { Process } \\
\text { Automation }\end{array}$ & $\begin{array}{l}\text { Wireless } \\
\text { medical } \\
\text { telemetry }\end{array}$ & $\begin{array}{l}\text { HW (operation } \\
\text { room surgical } \\
\text { monitoring) }\end{array}$ & $\begin{array}{l}\text { HW (WLAN } \\
\text { IEEE } 802.11 \mathrm{~b} / \mathrm{g} / \mathrm{n} \text { ) }\end{array}$ & $\begin{array}{l}\text { Indoor (multiple } \\
\text { rooms separated } \\
\text { by walls) }\end{array}$ & $\begin{array}{l}\text { Health } \\
\text { database } \\
\text { (MySQL) }\end{array}$ & $\begin{array}{l}\text { WLAN } \\
\text { signal } \\
\text { analyzer, } \\
\text { JPerf }\end{array}$ & scripts & $\begin{array}{l}\text { Database } \\
\text { requests }\end{array}$ \\
\hline $\begin{array}{l}\text { Fink } \\
2013[16]\end{array}$ & Robotics & Robot teams & $\begin{array}{l}\text { HW (mobile } \\
\text { AGVs) }\end{array}$ & HW (IEEE 802.15.4) & $\begin{array}{l}\text { Indoor (two } \\
\text { office/lab } \\
\text { buildings) }\end{array}$ & $\begin{array}{l}\text { Location } \\
\text { reported } \\
\text { at } 5 \mathrm{~Hz}\end{array}$ & $\begin{array}{l}\text { Signal } \\
\text { strength } \\
\text { reported } \\
\text { at } 5 \mathrm{~Hz}\end{array}$ & scripts & No \\
\hline $\begin{array}{l}\text { Liang } \\
2019 \text { [17] }\end{array}$ & $\begin{array}{l}\text { Factory } \\
\text { Automation }\end{array}$ & AGV, safety & $\begin{array}{l}\text { HW (mobile } \\
\text { AGVs) }\end{array}$ & HW (WIA-FA) & $\begin{array}{l}\text { Indoor (industrial } \\
\text { sites) }\end{array}$ & $\begin{array}{l}\text { Device } \\
\text { logging, } \\
\text { field } \\
\text { measures }\end{array}$ & $\begin{array}{l}\text { Signal } \\
\text { analyzer, } \\
\text { spectrum } \\
\text { analyzer, } \\
\text { network } \\
\text { analyzer }\end{array}$ & scripts & $\begin{array}{l}\text { Motion } \\
\text { distance } \\
\text { error }\end{array}$ \\
\hline $\begin{array}{l}\text { Candell } \\
2015[18]\end{array}$ & $\begin{array}{l}\text { Process } \\
\text { Automation }\end{array}$ & $\begin{array}{l}\text { Chemical } \\
\text { process } \\
\text { control }\end{array}$ & $\begin{array}{l}\text { HIL (process } \\
\text { simulator, PLC, } \\
\text { sensors) }\end{array}$ & $\begin{array}{l}\text { HW (IEEE } \\
802.15 .4-T D M A)\end{array}$ & Indoor (Lab) & $\begin{array}{l}\text { Simulator } \\
\text { logging }\end{array}$ & No & scripts & $\begin{array}{l}\text { Process } \\
\text { control }\end{array}$ \\
\hline $\begin{array}{l}\text { Liu, Y. } \\
2016 \text { [19] }\end{array}$ & $\begin{array}{l}\text { Process } \\
\text { Automation }\end{array}$ & $\begin{array}{l}\text { Chemical } \\
\text { process } \\
\text { control }\end{array}$ & $\begin{array}{l}\text { SW (process } \\
\text { simulator) }\end{array}$ & $\begin{array}{l}\text { SW (IEEE } \\
\text { 802.15.4-TDMA) }\end{array}$ & $\begin{array}{l}\text { SW (PER-SNR } \\
\text { curves) }\end{array}$ & $\begin{array}{l}\text { Simulator } \\
\text { logging }\end{array}$ & $\begin{array}{l}\text { Simulator } \\
\text { logging }\end{array}$ & scripts & $\begin{array}{l}\text { Process } \\
\text { control } \\
\text { safety }\end{array}$ \\
\hline
\end{tabular}

Various industrial scenarios have been considered to address the featured operational and environmental factors in the production site. Table 1 summarizes recent efforts on building testbeds for industrial wireless communications. Details of the comparison can be found in our earlier publication [7]. A work-cell, serving as the atomic production unit in modern manufacturing factories, has been identified as a promising industrial communication scene to observe the impact of communications on factory operations. Besides, new techniques can be introduced into measurements to improve the accuracy of data capture and facilitate data processing and analysis. Among them, time synchronization protocols and database techniques are examples that are playing a very important role. The former, 
e.g., Network Time Protocol (NTP)) and Precision Time Protocol (PTP), equips measurement devices with the tuned clocks synchronized to the same time server so that the captured local events can be realigned in a single timeline for the global view of various events and their interactions with each other [20]. The latter, e.g., GDBs, enables advanced data management schemes to organize heterogeneous measurement records and facilitate data exploration to get insights of CPS operations [7]. Some of the design and measurement work has been presented in our earlier publications [5-7]. This report serves as a reference document to provide more details in constructing the testbed and enabling the data workflow for further evaluation efforts.

\section{Overview of the Testbed Architecture}

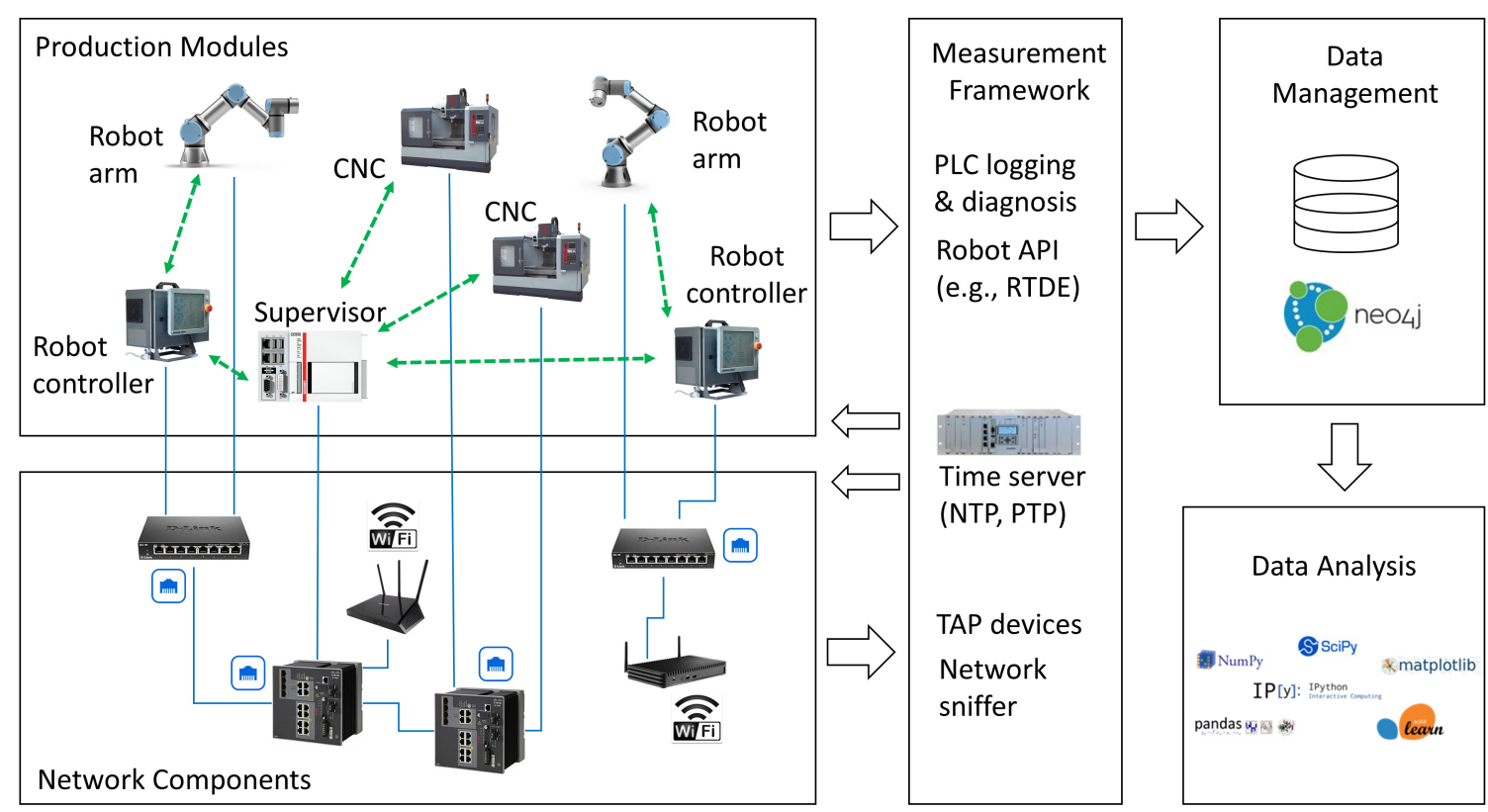

Fig. 1. The NIST industrial wireless testbed architecture

The NIST industrial wireless testbed is a work-cell level evaluation platform which generates typical communication sessions between production modules and enables close examination of transmission performance in communication links using wired or wireless technologies. As shown in Fig. 1, the architecture consists of subsystems including production modules, network components, a measurement framework, and data tools for management and analysis. Production modules are components of the work-cell manufacturing system that participate in job-oriented operations, such as order processing, task scheduling and assignment, tooling procedures, and machine tending. Network components are networking devices and the communication infrastructure, if there is any, on the factory floor. They are used to connect production modules to each other and enable their communications for information exchange and process control within and beyond work-cell 
fences. These two subsystems can form various industrial system use cases in the testbed experiments with corresponding configurations. The measurement framework probes into selected objects and connections to collect data, such as manufacturing progress, machine status, link quality, and network health. Rounds of experiment data will be transferred to a centralized data management system which manages records from heterogeneous sources and of different formats. We can utilize data analysis tools to explore the stored data for insights of the production process, the underlaying network, and their correlation. In the designed architecture, individual subsystems are connected by predefined interfaces and integrated as a complete evaluation process. Design details of each one will be elaborated in the following sections.

\section{Production Modules}

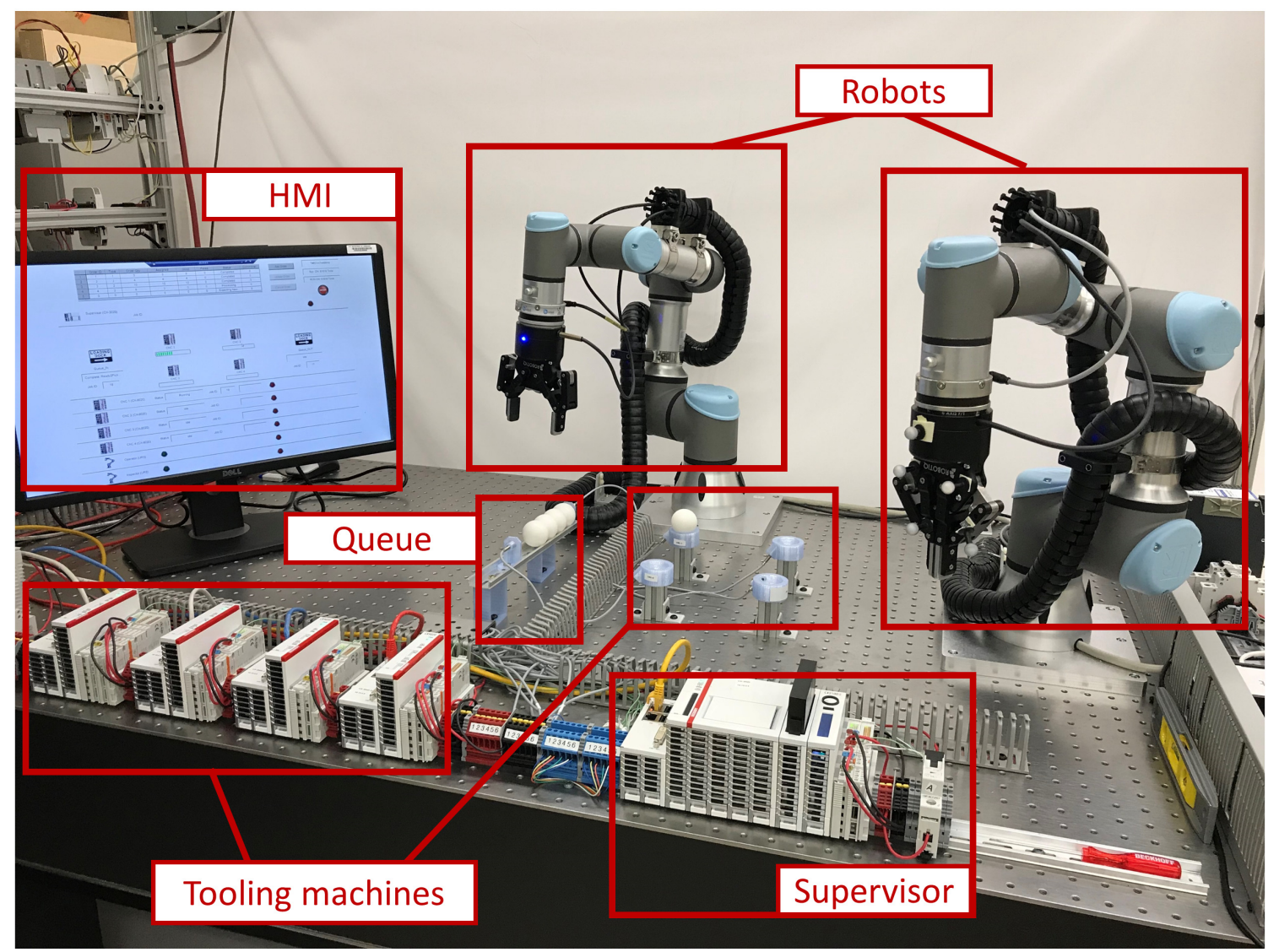

Fig. 2. Product modules in the testbed

In the NIST testbed, production modules are identified as the work-cell supervisor, tooling machines, collaborative robots, job buffers, and human-machine interface (HMI) according to their roles in the production process. Fig. 2 shows a snapshot of production modules in the testbed. 
In this section, we first specify the generic production workflow to introduce all the modules. Then, each module will be addressed with its own operation features and design details, such as the components, state machine, and module interface.

\subsection{Work-Cell Workflow}

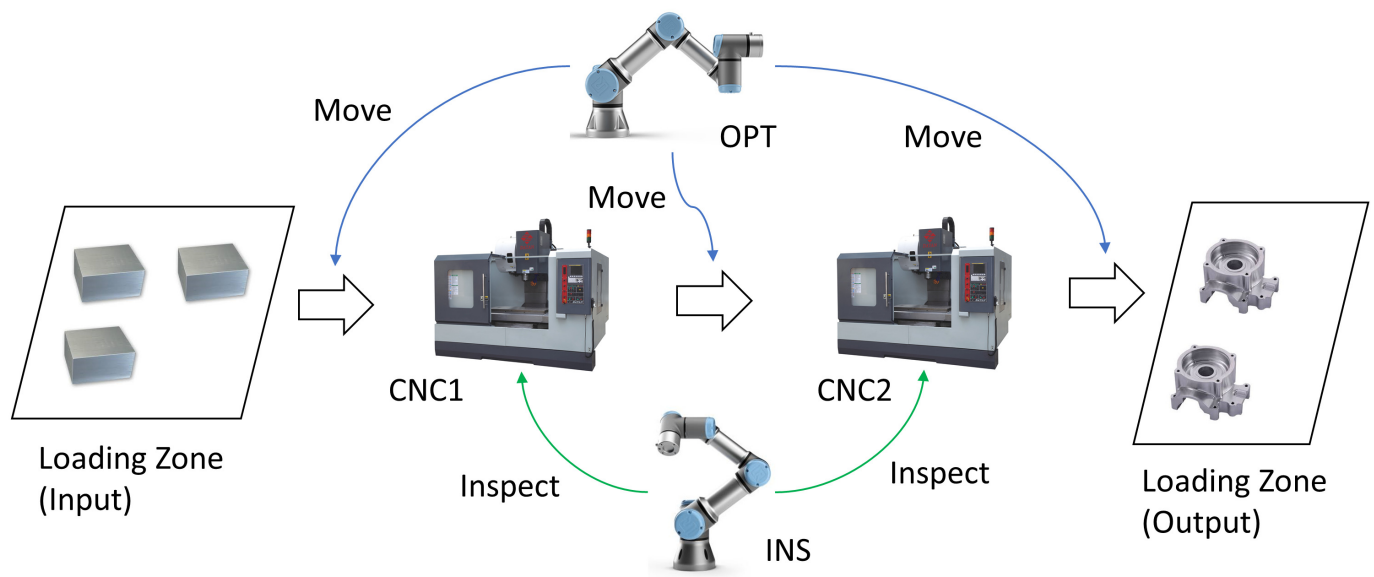

Fig. 3. Illustration of work-cell interactions in a tooling path

Based on available modules, we can develop many different production scenarios in a typical work-cell. In the first release of this report, the testbed is set to reproduce a simple, but typical, work-cell operation with collaborative robots, i.e., the machine tending use case. In the following discussions, we will focus on design issues of realizing this use case in the testbed. Specifically, work orders for the work-cell are submitted through the HMI. The supervisor adds received orders into a queue and processes them in the order assigned by the priority rule. Currently, it adopts the simple "first come, first served" policy. An order contains a batch of parts to be processed and the work plan specifying the tooling path, i.e., how each part moves through the work-cell until it is completed. The manufacturing job on a part can be one or multiple procedures performed in CNC machines, such as drilling, welding, painting, and heat treatment. Each machine is referred to as a job stop in the tooling path. Loading zones, or job buffers, are also treated as independent job stops where parts are temporarily stored waiting for the transfer to next procedure. There are two robots in the work-cell having different jobs in the production: one as the operator (OPT) and another as the inspector (INS). OPT moves parts between job stops; INS checks the part quality after each machine task and reports the inspection result to the supervisor. CNC machines and robots are the job-level actuators. They all rely on the supervisor for instructions of the next move. The supervisor, on its own, handles work orders, schedules operations of machines and robots, and monitors the whole workcell status. Fig. 3 illustrates interactions in a simple two-stop tooling path for a single part 
production. The repeated operations provide ample opportunities to collect statistically significant metrics of both the network and the operation of the work-cell.

\subsection{Supervisor}

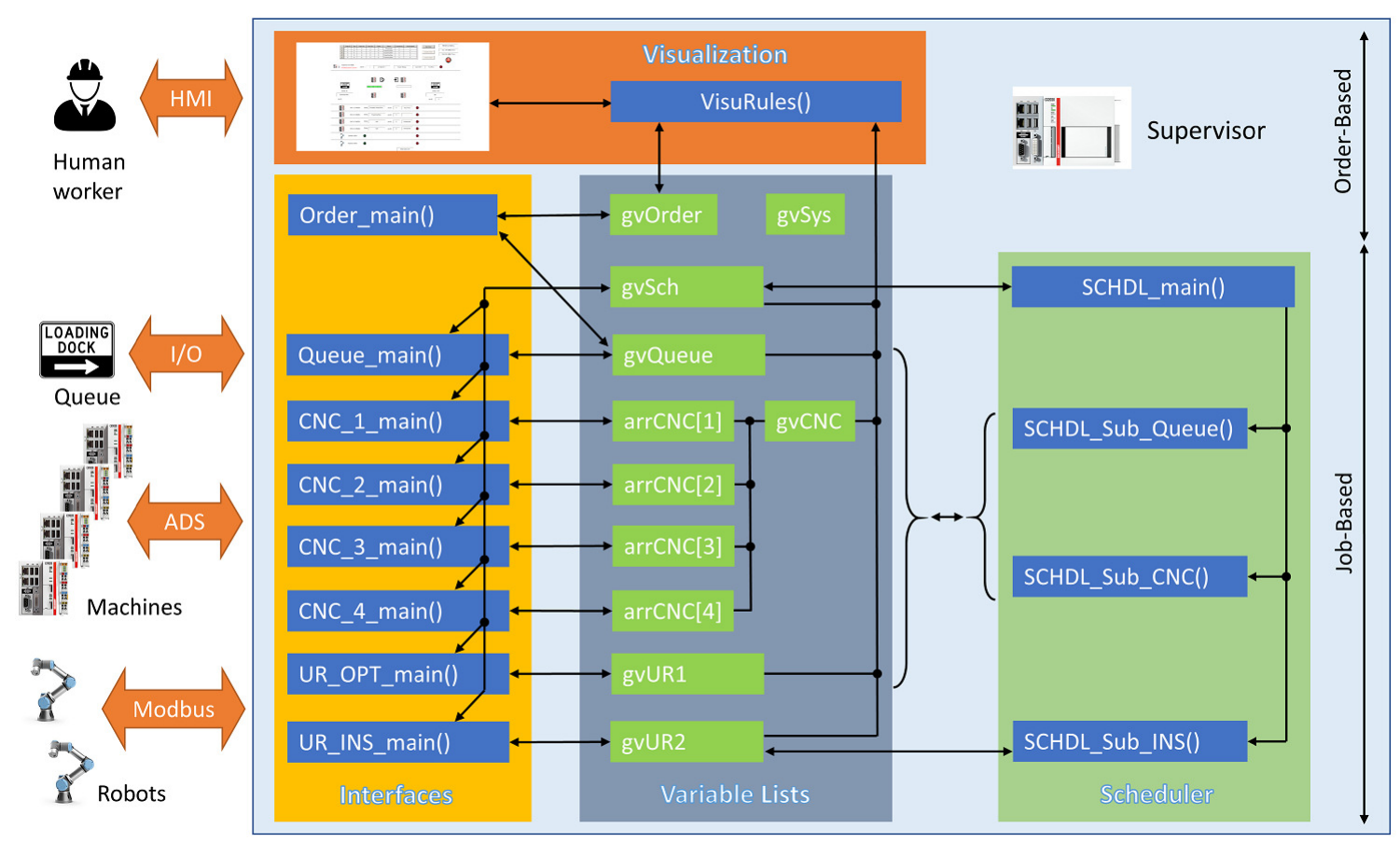

Fig. 4. Function modules in the supervisor PLC. Subsystem functions/programs are in BLUE, and global variables are in GREEN.

In the work-cell, the supervisor plays the role of a "brain". It interacts with upper-level manufacturing managers on behalf of the entire work-cell and manages the equipment, input materials, and output products in its coverage. Along the part's tooling path, as shown in Fig. 3, the supervisor keeps monitoring and manipulating all job stops and robots that are involved in individual procedures following the work plan. To fulfill its role as the central controller in the production, we identify the main functions of the supervisor: production scheduling, interfaces to upper management units or human workers (known as "northbound" interfaces), interfaces to field components of the work-cell (known as "southbound" interfaces), and inventory management.

The supervisor of the testbed is deployed in a Beckhoff PLC of the model CX2020. Accordingly, these functions are implemented in the PLC as shown in Fig. 4. To deal with different work-cell objects, a modular design is used to manage the PLC's memories, computing power, and interfaces. Four major modules defined in the supervisor PLC are the scheduler (SCHDL), global variable lists (GVL), interfaces, and visualization. SCHDL makes actuation decisions based on real-time equipment status and production progress. 
When a decision is made, the PLC will generate command messages and send them out through the interfaces to corresponding external actuators. Among Interfaces, the northbound connects to upper-level factory entities, such as manufacturing execution systems (MES) and enterprise resource planning (ERP) systems, dealing with incoming orders and status reporting; the southbound, on the contrary, is linked with field devices including machines, robots, and job buffers. The system-wide data sharing between function modules takes place in GVL, e.g., caching machine status and control decisions exchanged between interfaces and scheduler. System variables associated with a specific function or work-cell component are managed in its GVL instance and are named accordingly, e.g., gvCNC contains four array objects each of which stores the relevant information of a CNC machine in the work-cell. In addition, the PLC also hosts a human-machine interface (HMI) in the visualization module for interactions with human staff.

From the perspective of information processing, functions and data memories in the supervisor can be divided into two planes: order-based and job-based. Order-based functions deal with incoming orders, update of the order status based on real-time production results, and maintaining the inventory. On the other hand, job-based functions mainly work on the associated work-cell components and coordinate their production activities following the schedule. Such a modular design allows the supervisor to easily adapt to the evolving work-cell tasks and utilize state-of-the-art techniques to leverage individual functions.

\subsubsection{Scheduler}

The supervisor is deployed in a Beckhoff's CX2020 PLC, which runs the TwinCAT 3 realtime engine in a Windows embedded standard (WES) 7 operating system. TwinCAT 3 guarantees determinism of PLC tasks with fixed cycling steps. The cycle time is set to $1 \mathrm{~ms}$ in the testbed. The scheduler function is called at the beginning of each cycle so that the scheduler checks system status and makes operation decisions at $1 \mathrm{kHz}$.

The supervisor implements a simple job scheduler which follows a few rules to work.

\section{- Rule 1: Single active order}

At any time, the schedule can treat one and only one order in the work-cell. Once an order is being processed, the other orders should be held until it is completed. When the scheduler is free for picking the next order, the selection criteria include, but not limited to, the order's priority (i.e., the primary factor) and its arrival time in the queue (i.e., the secondary factor). Since multiple parts in an order can be processed at different $\mathrm{CNC}$ machines simultaneously, the scheduler has to manage production components and coordinate their actions in the parallel tasks given the work-cell's capacity. For example, if the order indicates a tooling path involving two machines, the scheduler can assign a new part to the machine of Step One after the finished part leaves for Step Two. Both machines can run at the same time working on parts in different procedures for the same order.

\section{- Rule 2: Single active robot}

Robots are moving objects in the work-cell. For safety reasons, only one robot is 
allowed to move in the work-cell at any time. In other words, the scheduler will lock one robot in its home position before enabling another robot to work. As shown in Fig. 3, the production progress is always related with robot moves, either a part transition between job stops (by OPT) or an inspection (by INS). Specified in this rule, each time the scheduler can only make one robot, either OPT or INS, work. We use the term of scheduling window to refer to the moment when the scheduler is free to choose the next move for the robots.

\section{- Rule 3: In-process parts first}

In-process parts are those who have started their tooling procedures in work-cell machines. In a scheduling window, in-process parts may have finished the work in current job stops and are waiting for the robot to either perform inspection or transfer to the next stop. Following Rule 2, they should be served first before any new parts. Of note, among all pending requests, intra-stop moves, i.e., inspection, are always prioritized. With multiple part move requests of the same type, the scheduler uses the round robin method to select the first one to serve in the queue.

As shown in Fig. 4, the scheduler's main function, SCHDL_main, is called to verify the scheduling window and look for the next move at the beginning of each cycle. Individual member functions will then create instructions to the selected machine and/or robot based on the scheduler's decision and cache them in the corresponding variable lists. In the same cycle, interface functions will check the saved variables and transmit the newly added scheduling information to external devices.

\subsubsection{HMI}



Fig. 5. Work-cell HMI and the control functions 
The work-cell HMI server is deployed in the supervisor PLC as shown in Fig. 5. HMI serves as the main northbound interface for external communications. HMI displays the work-cell status collected by the supervisor in multiple windows. Fig. 5 shows the main window during the operation. Transitions to the other HMI windows provide additional configuration options other than the default, e.g., measurement setup. Human workers can remotely monitor and interact with the automated production process in specific windows, such as placing orders and stopping/resetting the production.

HMI has its own PLC task thread in Beckhoff CX2020 whose priority is lower compared to the job scheduler. The main visualization control function, VisuRules, is called every $200 \mathrm{~ms}$ to refresh the view. It reads the GVL variables, such as $g v S c h$ for the scheduler, and converts values to strings or Boolean values for respective display objects. It uses an independent function, VisualControl_Order, to handle any update in the online order editor of the main window. VisualControl_Order interacts with gvOrder in GVL to add, remove, and update orders in the work-cell.

HMI can be accessed directly through the supervisor PLC. The PLC runs a customized Windows operating system and has fully functioned computer interfaces, such as a video output and USB ports, HMI can be displayed in a screen connected to the PLC and operated by input devices such as a mouse. Thanks to the Windows remote desktop connection, we can also access the work-cell HMI through a remote host that resides in the testbed's LAN.

\subsubsection{Job Buffers}

Job buffers are the temporary storage for parts before or after the production which is comprised of two loading zones in the work-cell, i.e., the input (Queue IN) and output (Queue OUT) buffers. They also serve as the start and end job stops in a tooling path for a single part, respectively. The tooling path can be in turn presented as a sequence of job stops, e.g., $<$ Queue IN $>-<C N C 1>-<C N C 3>-<$ Quеие OUT $>$. In the testbed, both input and output job buffers are implemented as subsystems in the supervisor and managed by the function Queue_main. The supervisor detects part arrivals/departures in the buffers with proximity sensors which are connected to the PLC's digital input module, one for each.

\subsubsection{Production Tasks}

Work-cell operations in the testbed are designed around three types of production tasks: orders, parts, and jobs. Orders are the basic unit of input assignments from upper-level management systems to a work-cell. An order contains quantities of the requested parts and their manufacturing specs. Parts move between different machines following the planned tooling path. A job is always associated with an actuator, i.e., a series of actions are taken by the actuator in treating the part. For example, a CNC machine serves to drill holes in the part, or a robot moves the part between the consecutive job stops. Elements used to describe a job include the commanding body (which assigns it), the execution body (which performs it), the treated part/object, action(s), and the time. 


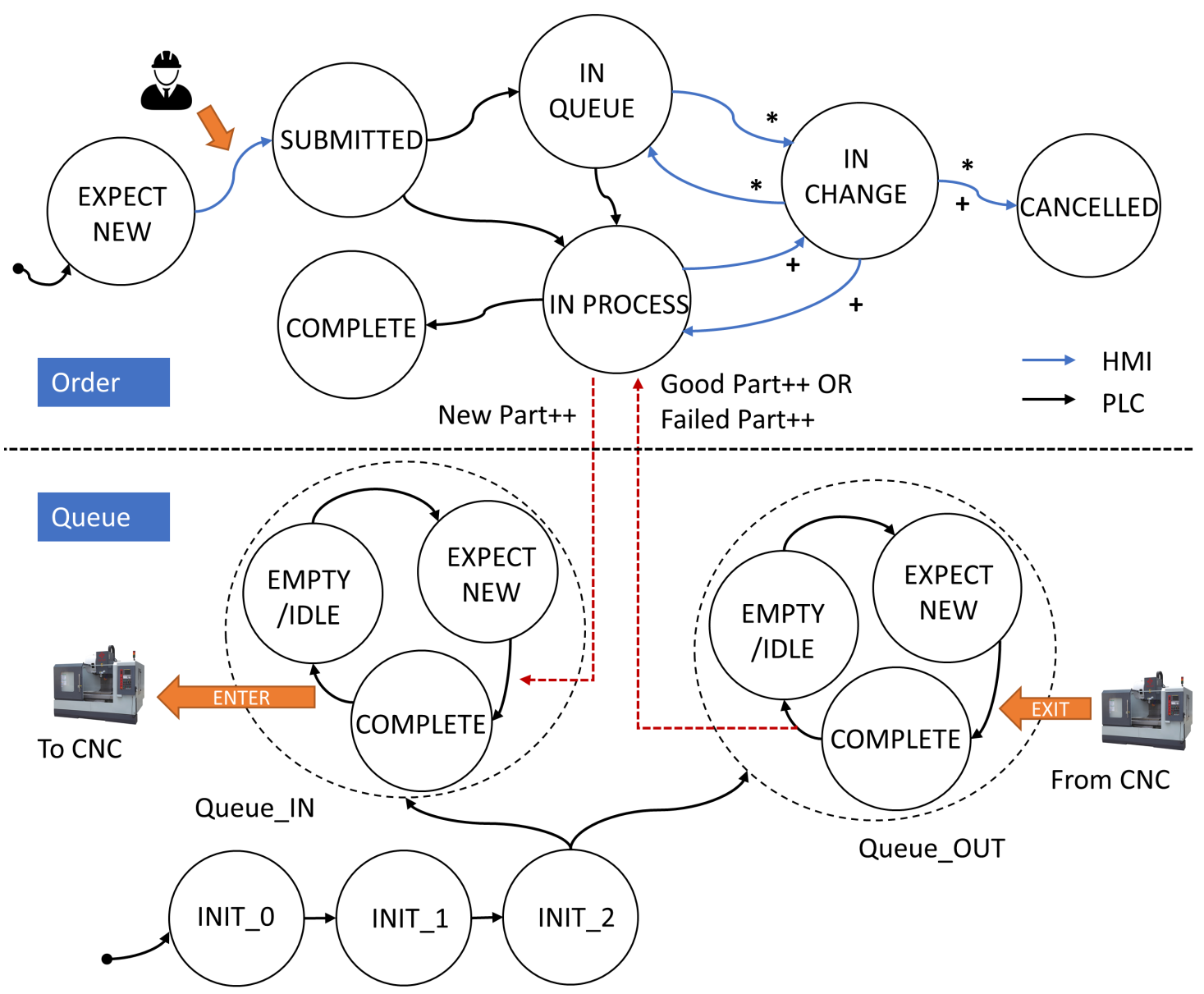

Fig. 6. State machines of orders and queues

In the work-cell, the supervisor is responsible for treating the order-related information. It receives orders of parts, assembles production instructions for them, and coordinates work-cell components in the production. At the supervisor, the order module and the queue module (which manages job buffers) have their own state machines, as shown in Fig. 6. The scheduler interprets an incoming order and identifies the part's tooling path in terms of a planned sequence of jobs at the selected work-cell actuators. Specifically, only the "IN PROCESS" order is loaded by the scheduler, i.e., one active order is in production. Each time when a new part is scheduled for the current order, the scheduler assigns a new job to the input buffer. The part production starts at the input buffer and moves on to the first tooling machine once it is available. The scheduler keeps tracking the part status until it finishes the last tooling task and arrives at the output buffer. Then, based on the inspection results, either a good product or a labeled failure is added into the order's record. Once the good part number is fulfilled, the order will move to the "COMPLETE" state. The queue module will start to process the next pending order by labeling its state to "IN PROCESS".

Interactions between the order module and the queue module, which are indicated as 


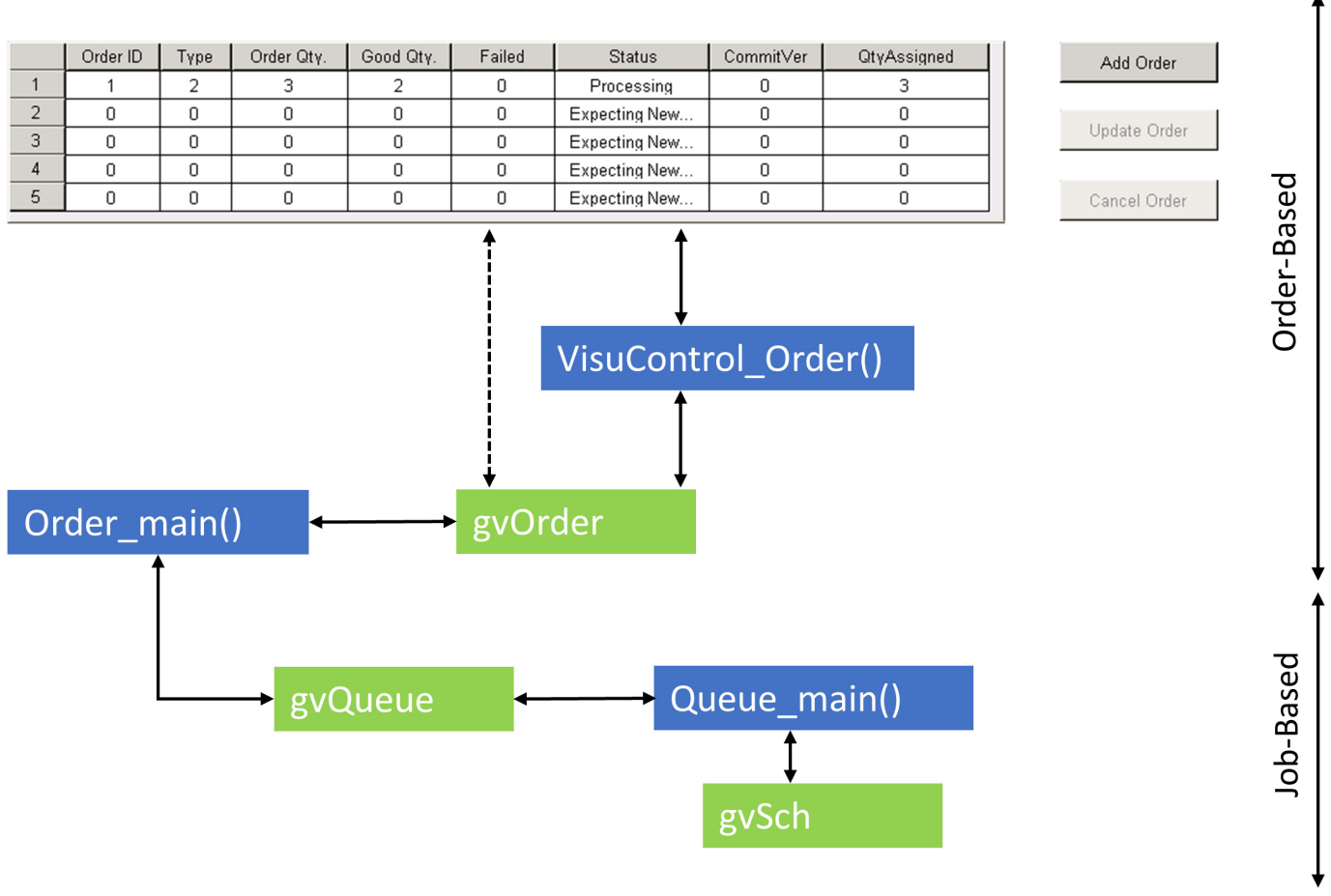

Fig. 7. The online order editor module in HMI

red dashed arrows in Fig. 6, are mainly through the exchange of variables in the GVL gvQueue shown in Fig. 7. All the updates in the order module are finally visualized in the HMI online order editor.

\subsection{Tooling Machines}

We consider four CNC machines as machining tools in the work-cell. Production processes for individual jobs in these machines are programmed and managed by their own controllers. The embedded CNC controllers communicate with the other work-cell members, mainly with the supervisor, for production coordination. Therefore, each CNC machine is emulated by a separate PLC in the testbed which focuses on characterizing its tooling behaviors and communication traffic patterns. Each CNC machine consists of a Beckhoff CX9020 PLC, a part holder, and a proximity sensor. The PLC mimics states of the emulated $\mathrm{CNC}$ machine in its tooling cycle and exchanges the instantaneous status and job information with the supervisor. The part holder represents the machine's working zone where the part is treated. The proximity sensor is placed in the holder to monitor the part arrival/departure and is connected to the PLC's digital input module.

The testbed is aimed to evaluate the correlation between data transmissions and workcell operations which impact the whole system performance. Therefore, the CNC emulator is focused mainly on mimicking the machine's behavior with time dependent and statistical 


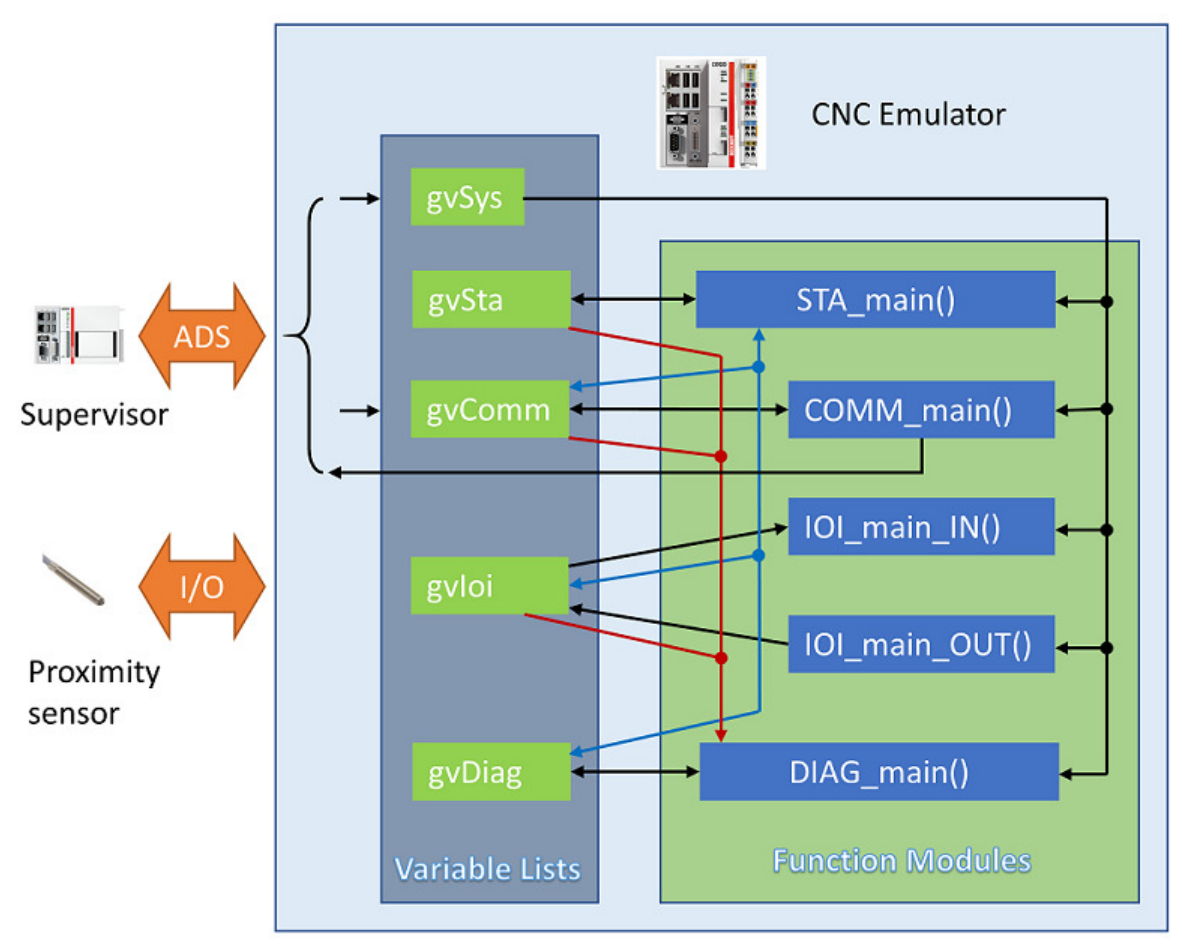

Fig. 8. Architecture of the CNC machine emulator

performance features, such as the production efficiency, error and downtime distributions, and part defects. Meanwhile, the emulators also help shape the work-cell data traffic with their periodic status updates and on-demand messages during the production.

Following the similar modular design in the supervisor, the CNC emulator also defines its function modules and GVL in the implemented PLC as shown in Fig. 8. Specifically, function modules include the state machine (STA), communications (COMM), I/O module interfaces (IOI) ${ }^{1}$, and diagnostics (DIAG). The shared system variables between modules are maintained in GVL, e.g., gvSta maintains variables related with the state machine and gvSys contains the system-wide information such as the machine's identification (ID) and network address.

To fully capture operational and communication activities of a machine tool, the CNC emulator conducts state-dependent operations and communications characterized by the state machine, as shown in Fig. 9. The state machine is serviced in the STA function module, which contains three main states: initialization (INIT), IDLE, and BUSY. Each main state contains a few substates, which characterize further details of operations. INIT along with its substates facilitate the synchronization among distributed nodes whose design will be discussed with more detail in Section 4.5.1. The substates of the "BUSY" state represent a series of machine operations regarding a single job. Using either embedded timers

\footnotetext{
${ }^{1}$ The IOI functions are further grouped into IOI IN and IOI OUT, respectively.
} 


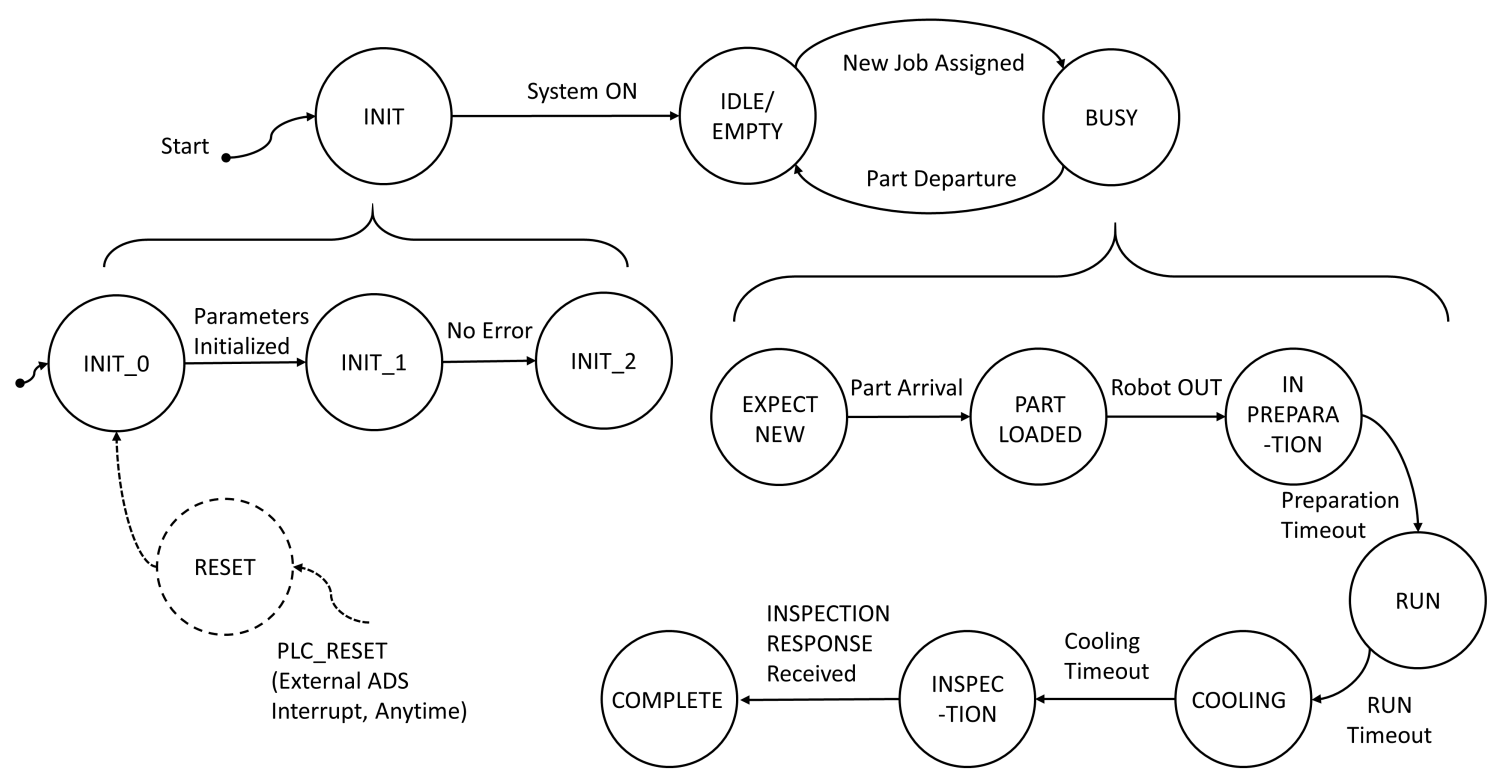

Fig. 9. CNC state machine

or external communication messages, the emulator triggers state transitions. The dwelling time in each (sub-)state can be either timed according to the machine's specification, e.g., the approximate G-code execution time and material removal rate, or determined by external events that trigger state transitions, e.g., a notification message. Randomness can also be introduced based on statistical machine/production models. Examples of randomness components in the models include: 1) the time of a tooling procedure; 2) time varying energy consumption in different states, e.g., power variations in material-drilling processes; 3) tool life estimation; 4) part defect rate; 5) measurement drift between calibrations; and 6) safety related events, e.g., unexpected interrupts due to object intrusion. Using empirical models and measurement data, we can model the above performance metrics statistically and regenerate the state-related traffic for the studied machine.

Therefore, the machines emulated in the testbed can be programmed to highlight the details of real practices to study the network impact on the work-cell performance. Process variables are modeled in the testbed by focusing on different topics such as 1) the production (task) efficiency, e.g., the execution time, material removal rate, energy consumption, and part defect rate; 2) asset health, e.g., the tool life time, failure probability, and downtime schedules for calibration and maintenance; and 3) work-cell collaboration, e.g., the clock drift, coordination precision, and safety. Besides checking the network support on routine data transmissions as scheduled, the testbed is particularly useful for testing the network performance in extreme cases with rare occurrences, e.g., the shutdown due to overheating or power surges. The machine emulator can produce the traffic in different use cases following the code, such as the recovery from unexpected overload events or in emergency 


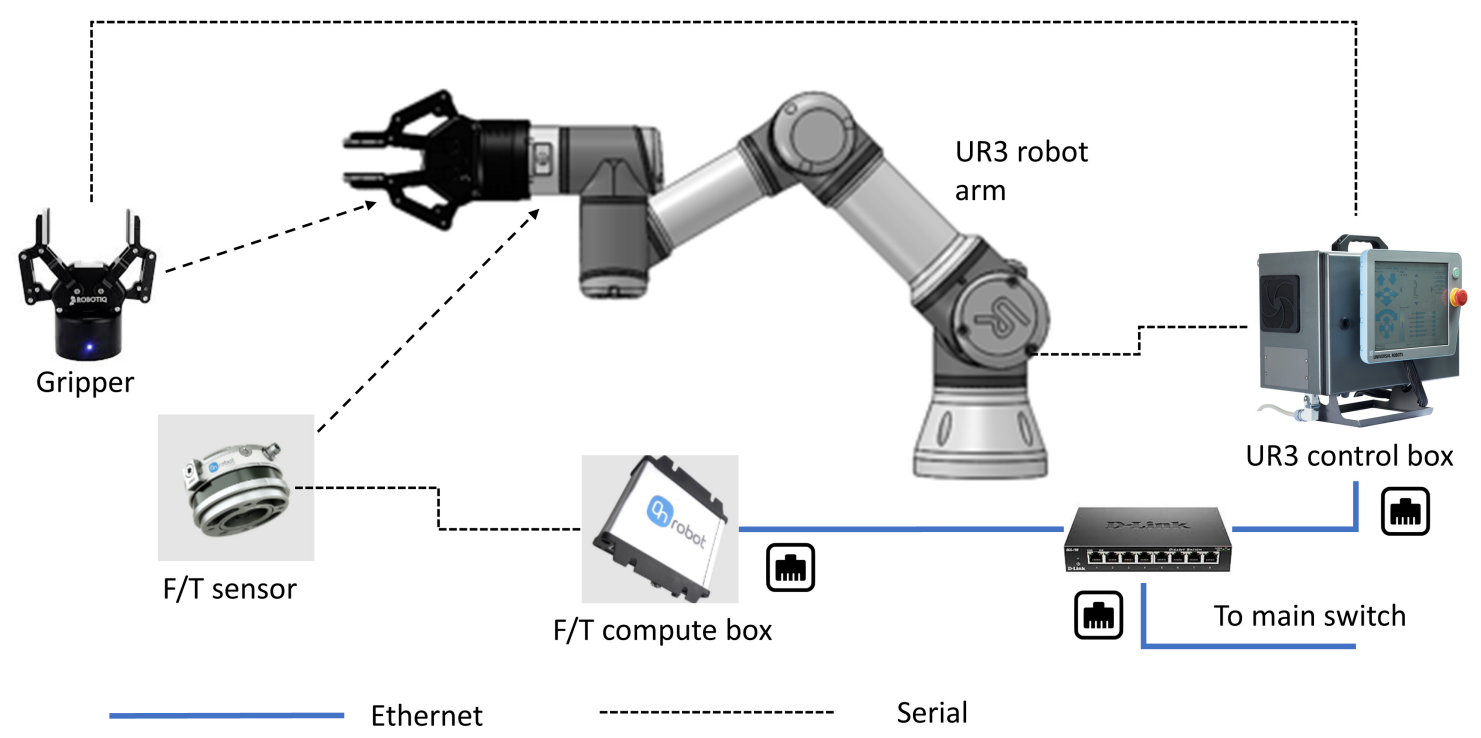

Fig. 10. A UR3 collaborative robot system

cases, and can repeat the case in multiple runs for conclusive experiments or comparative studies.

The quality of "product" in each job stop is also virtually rendered in the testbed. Each part quality in a machining process is randomly determined following a statistical model that mimics the typical defect rate in a real machine. The result is made in the inspection phase which allows the supervisor to schedule the next move accordingly. According to the study of the quality and quantity relationship in production systems [21, 22], part failures are associated with both independent and dependent factors. Independent failures usually follow a Bernoulli distribution with the uncertainty of temporal independence. On the other hand, dependent types of failures, which are often referred to as "persistent" or "systematic" ones, are those caused by tool failures, such as the broken drill or clog in the painting tube. In such cases, the failure of product is highly related with the asset failure rate. Since both types of failures are intuitively decoupled, the testbed carries product failures as well as the ones in assets to emulate occurrences of exceptions across time. Delivery delay or loss in communication links also affect operation performance and safety measures.

\subsection{Robots}

The testbed hosts two Universal Robots UR3 CB-series robots that perform machine tending in the work-cell. As shown in Fig. 10, each UR3 system consists of a 6 degree-offreedom (6 DoF) robot arm, a controller in the control box, and extended UR capabilities (URCaps) which are accessories including a gripper and a 6-axis force torque (F/T) sensor. The F/T sensor is further divided into two parts: the sensor unit attached to the end effector and a compute box connected to the UR3 control box. The UR3 provides a graphical user interface (GUI), called Polyscope, which serves as the main programming interface in the 


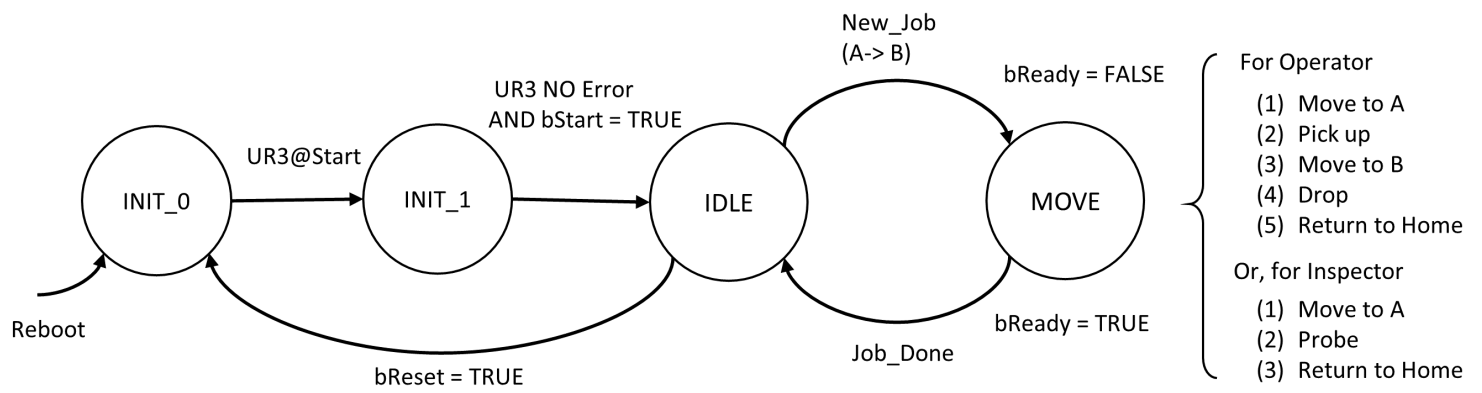

Fig. 11. The state machine of the UR3 robot in the work-cell

teach pendant. In Polyscope, users can input actuation commands and configure waypoints using programming wizards. In addition, users can also use the URScript programming language to assemble scripts for the robot control. In the control box, the UR3 controller interprets user input to plan the trajectory of the robot arm and dispatches set points to microcontrollers of individual joints at $125 \mathrm{~Hz}$. At the same rate, each microcontroller manipulates the corresponding joint with the calculated force and torque.

The controller also acts as the robot's communication portal to the work-cell. Robots receive actuation commands from the supervisor and report their status back. In the workcell, the OPT's job of transiting parts can be translated into a sequence of actuation commands in the UR3 script: (1) moving to waypoint A (from the Home position), (2) picking up the part at A, (3) moving to waypoint B, (4) unloading the part at B, and (5) returning to the Home position. Setting profiles of actions, such as arm positions at individual waypoints, are stored in the controller and loaded into the program once it starts. Similarly, INS's actions in a job include (1) moving to waypoint A (from the Home position), (2) probing the seated part at A and generating an encoded inspection result, and (3) returning to the Home position.

To interplay with the supervisor/scheduler and machines in the work-cell, the robot's state machine is also divided into two phases: initialization and operation, as shown in Fig. 11. During initialization, the robot walks through INIT_0 and INIT_1 following instructions from the supervisor. In $I N I T_{-} 0$, it resets all internal parameters and returns to its Home position out of the working space; in INIT_1, the robot exchanges its status with the supervisor and gets ready for any new operation. In the normal operations, the robot switches states between IDLE and MOVE while reporting the supervisor about its real-time status through the network.

The UR3 performs a series of status checks at the beginning of its control cycle and takes actions correspondingly given the state machine. Fig. 12 illustrates the major steps and branches in a typical control cycle.

\subsection{Coordination between Work-Cell Modules}

Since work-cell components are collaboratively working in the production, the testbed implements multiple approaches to coordinate these distributed nodes. 


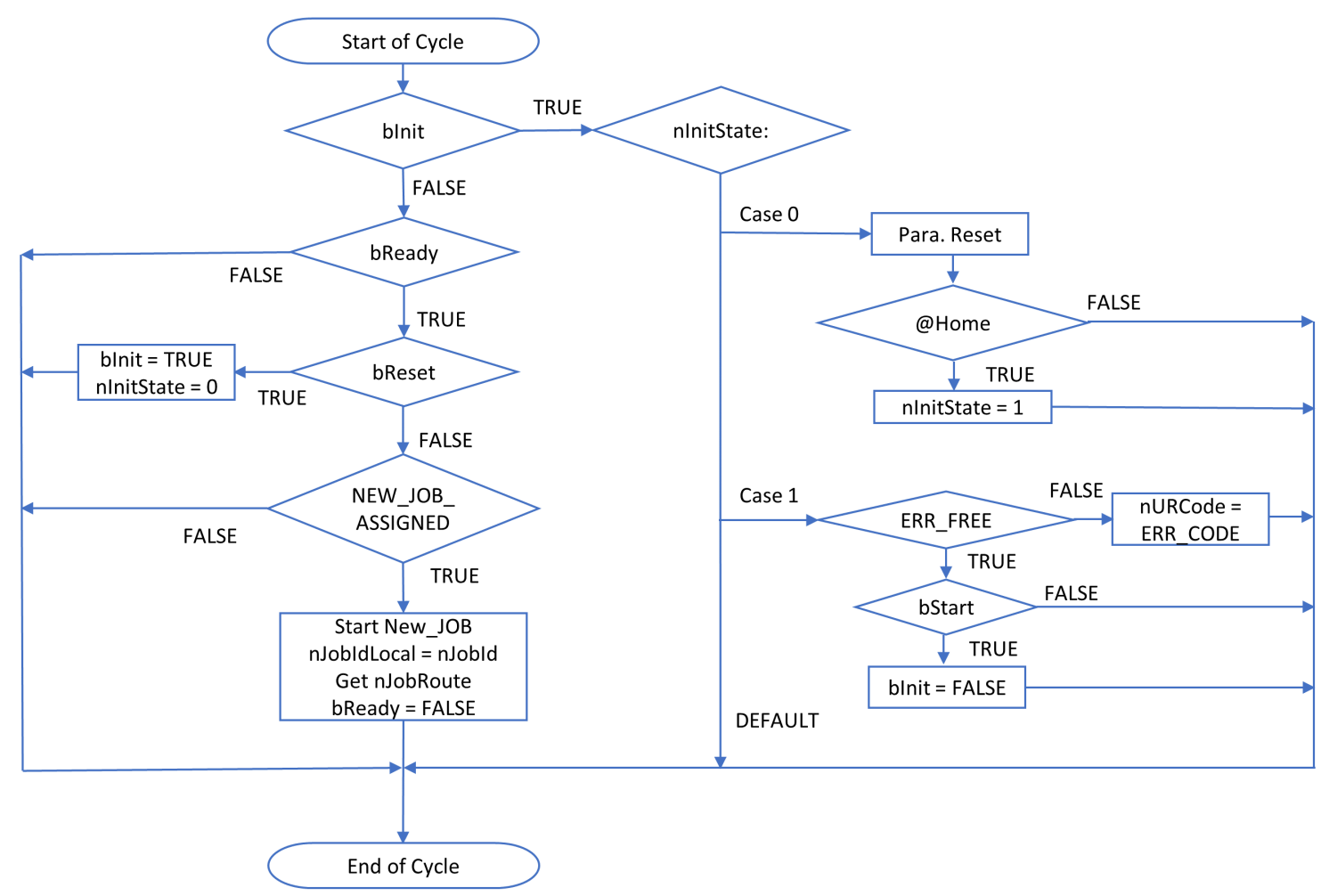

Fig. 12. The flow diagram in a UR3 robot cycle 


\subsubsection{Coordination in Initialization}

At the beginning of the testbed experiment, after powering on all components, a phased initialization process is performed where three steps are taken sequentially:

- INIT_0 Parameter initialization/reset,

- INIT_1 Logic error check and confirmation, and

- INIT_2 Loading ready-to-go state.

The scheduler in the supervisor provisions the whole initialization process over the work-cell. Fig. 13 illustrates such a process within the supervisor and other remote production modules. Red dashed lines refer to state switches triggered by messages. Specifically, the scheduler triggers its own state transitions right after it receives notifications from the other sub-modules in the supervisor that acknowledge the completion of designated procedures in the current phase. As shown in Fig. 13, the triggering events are denoted by blue curved arrows. The scheduler will then dispatch signals in the supervisor to trigger transitions in the internal sub-modules (shown in red curved arrows). For production modules in the field and their corresponding interfaces in the supervisor, messages carrying status information of the counterpart can serve to trigger internal state transitions. For example, the $\mathrm{CNC} \mathrm{x}$ interface needs the remote $\mathrm{CNC} \mathrm{x}$ machine to confirm the reception of restart command before it switches from INIT_O to INIT_I. In another case, remote modules wait for the interfaces to set a start flag before it completes INIT_2 and starts normal operation. In both cases, messages are denoted by green curved arrows in the illustrated process which serve as a necessary condition in state transitions. In INIT_2, remote modules first enter the idling mode getting ready for optional commands from the supervisor. Then the supervisor's sub-modules enter the ready mode coordinated by the scheduler. Once the entire testbed is ready, the production starts from the first order placed into the order module.

Thanks to the introduced initialization process, a "soft" online reset scheme is implemented in the testbed. Any time when clicking the "reset" button in the HMI, we can gracefully stop the ongoing experiment and reset the whole work-cell. Once reset is flagged, the scheduler can detect it at the beginning in the next PLC cycle (in $1 \mathrm{~ms}$ or less). It dumps all remaining orders in the queue, clears working status, and restarts from INIT_0. As shown in Fig. 14, starting from the scheduler, a system-wide reset will first expand from the scheduler to all internal modules in the supervisor, then reach out to individual work-cell modules through the established interfaces. 


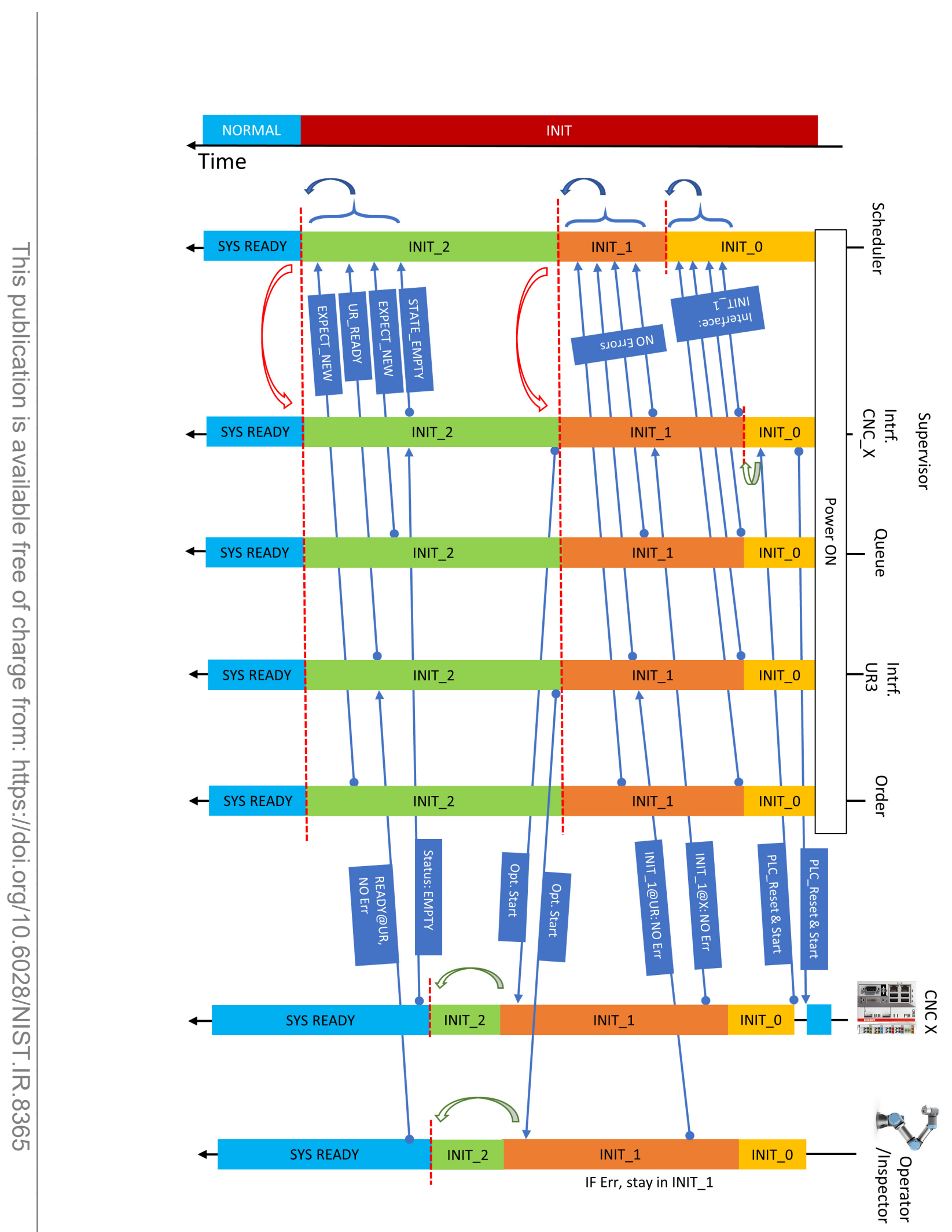

Fig. 13. Timeline of initialization steps. 


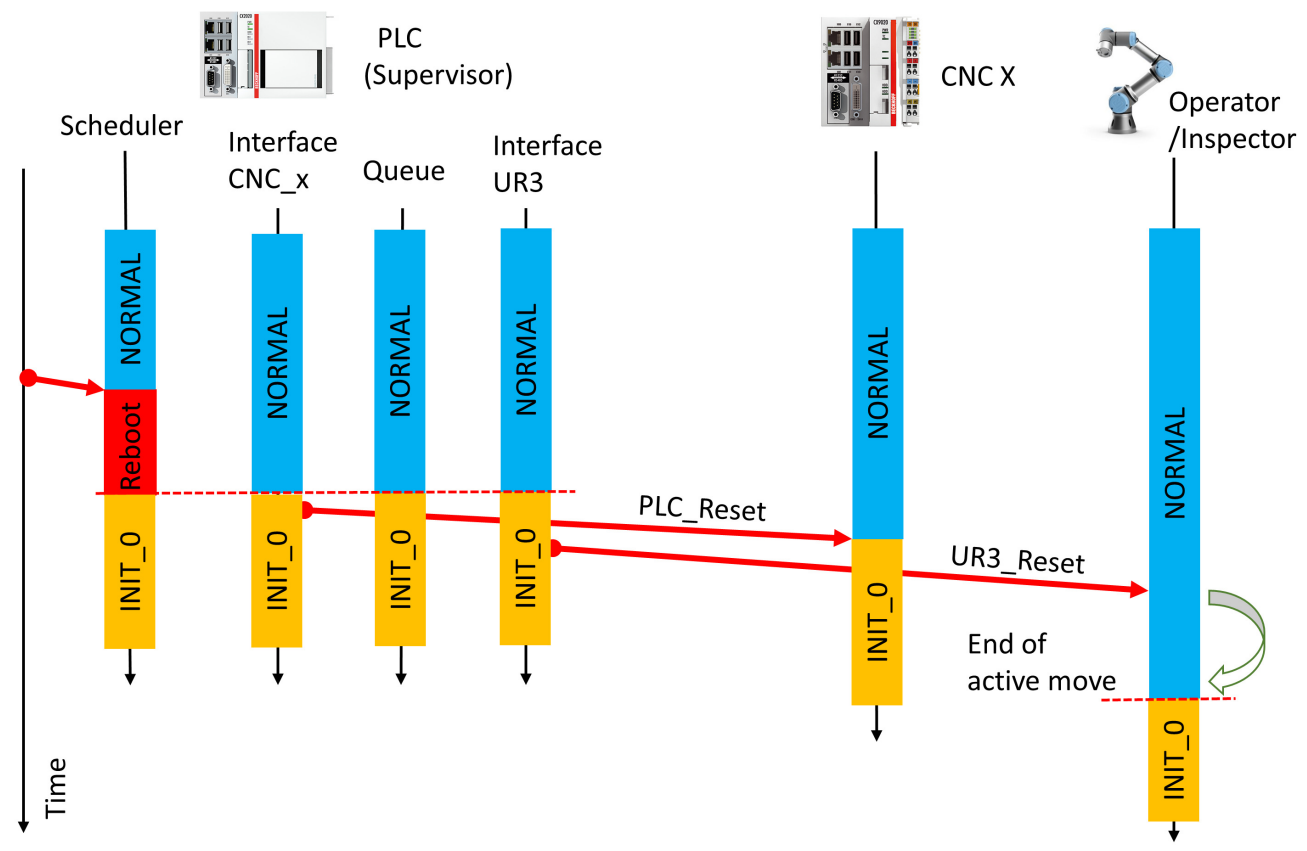

Fig. 14. Reset coordination between work-cell modules

\subsubsection{Coordination in Job Operations}

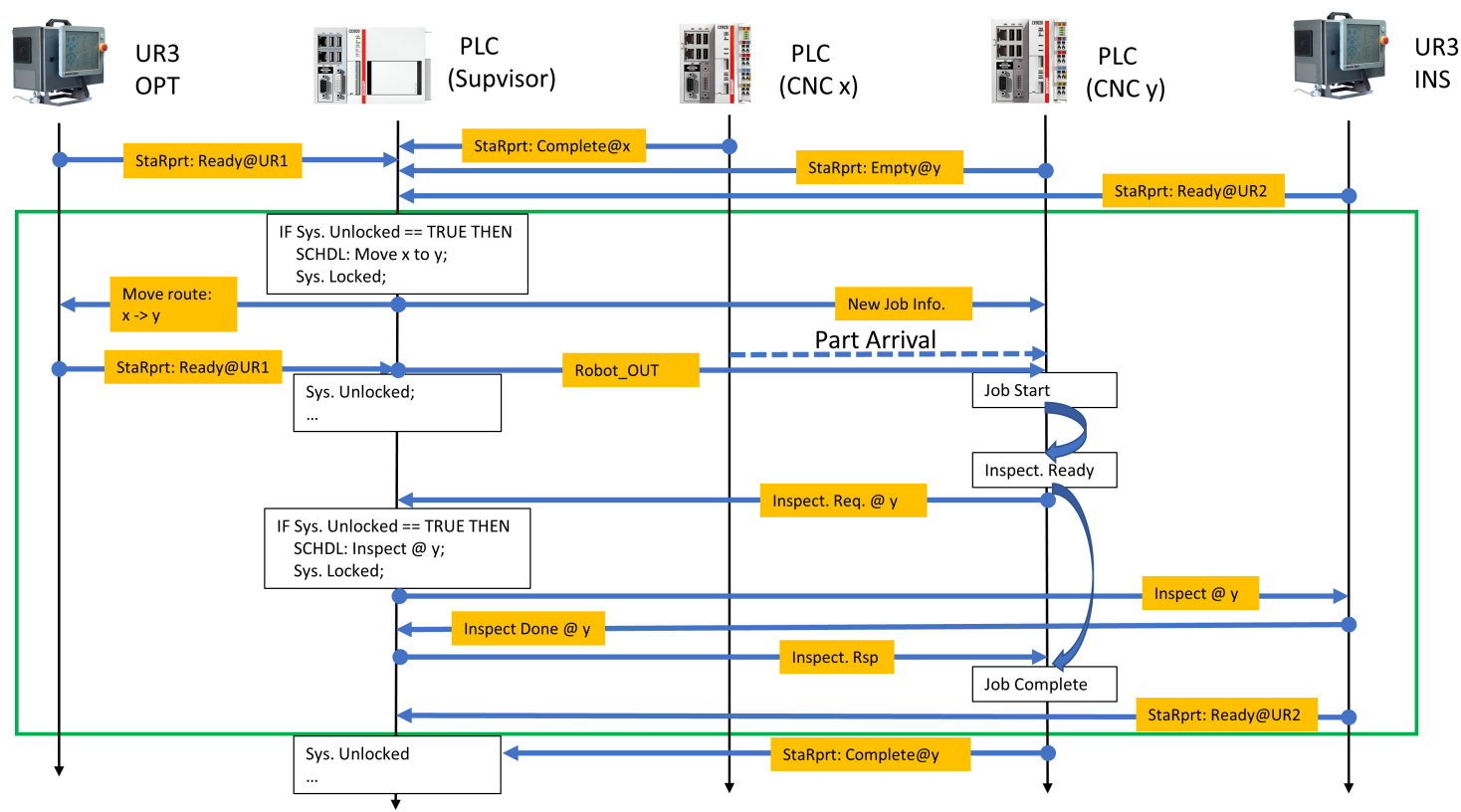

Fig. 15. Timeline of the coordination in an intermediate tooling procedure

Multiple production modules are involved in the tooling path. Recall the workflow as 
introduced in Section 4.1, to complete the part processing in a single machine, it needs the supervisor, both robots, and the machine to work together. The coordination between actuators becomes necessary to fulfill production operations and guarantee the safety. Fig. 15 illustrates the timeline of such coordination in a machining procedure.

Testbed operations are designed as a fully automated process, which needs no physical contact with parts or actuators by human staff in the production. Workers are anticipated to manage the work-cell through the HMI remotely. Possible collision risks are only related with part-oriented interactions between robots and running machines, e.g., when OPT unexpectedly approaches to a part that is being treated in a fast running CNC. Without wellestablished safety rules for work-cell operations, such incidents may interrupt the ongoing production, or even worse, cause asset damages that brings the work-cell down. Therefore, we have introduced multiple safety approaches in the job coordination to eliminate risks and protect the assets. First, the supervisor implements a safety flag in its scheduler to indicate whether an active robot is moving in the work-cell. It complies with the scheduling Rule 2, i.e., at most one robot is actively operating. Once the flag is set, the locked scheduler would not assign a new job to another robot so that collisions between robots are avoided. Second, the moving robot keeps notifying the machine(s) that may be affected by its maneuvers in the active job trajectory. The machine(s) on watch would not start to process the part until the robot returns to its Home position, such that it leaves a safe space for tooling operations. As shown in Fig. 15, the "Robot OUT" message clears such watch after the acting robot finishes its job. In addition, a logic check is performed at the robot on the waypoint instruction sent from the scheduler, which prevents the robot from operating some out-of-date instructions due to transmission failures. Preliminary experimental results indicate that the introduced approach supports collision-free operations in the testbed through very light coordination, as low as $1 \mathrm{~Hz}$, in the supervisor-robot link.

In the work-cell, there are two main interaction scenarios, part transition and inspection, both of which involve multiple machines with different jobs in the respective processes. Fig. 16 and Fig. 17 illustrate these two cases, respectively.

The main interactions between work-cell (sub-)modules in a part transition scenario are listed as follow,

- [A (START)] CNC x just finishes the part processing and changes its status to "COMPLETE";

- [B] CNC x updates its status to the supervisor via routine status report in its ADS connection to the supervisor;

- [B1] The COMM module in CNC x loads current status and formulates a status report triggered by self timer;

- [B2] The COMM module sends the status report to the supervisor (ADS Write);

- [C] The scheduler in the supervisor receives the status and updates it in the machine status register; 


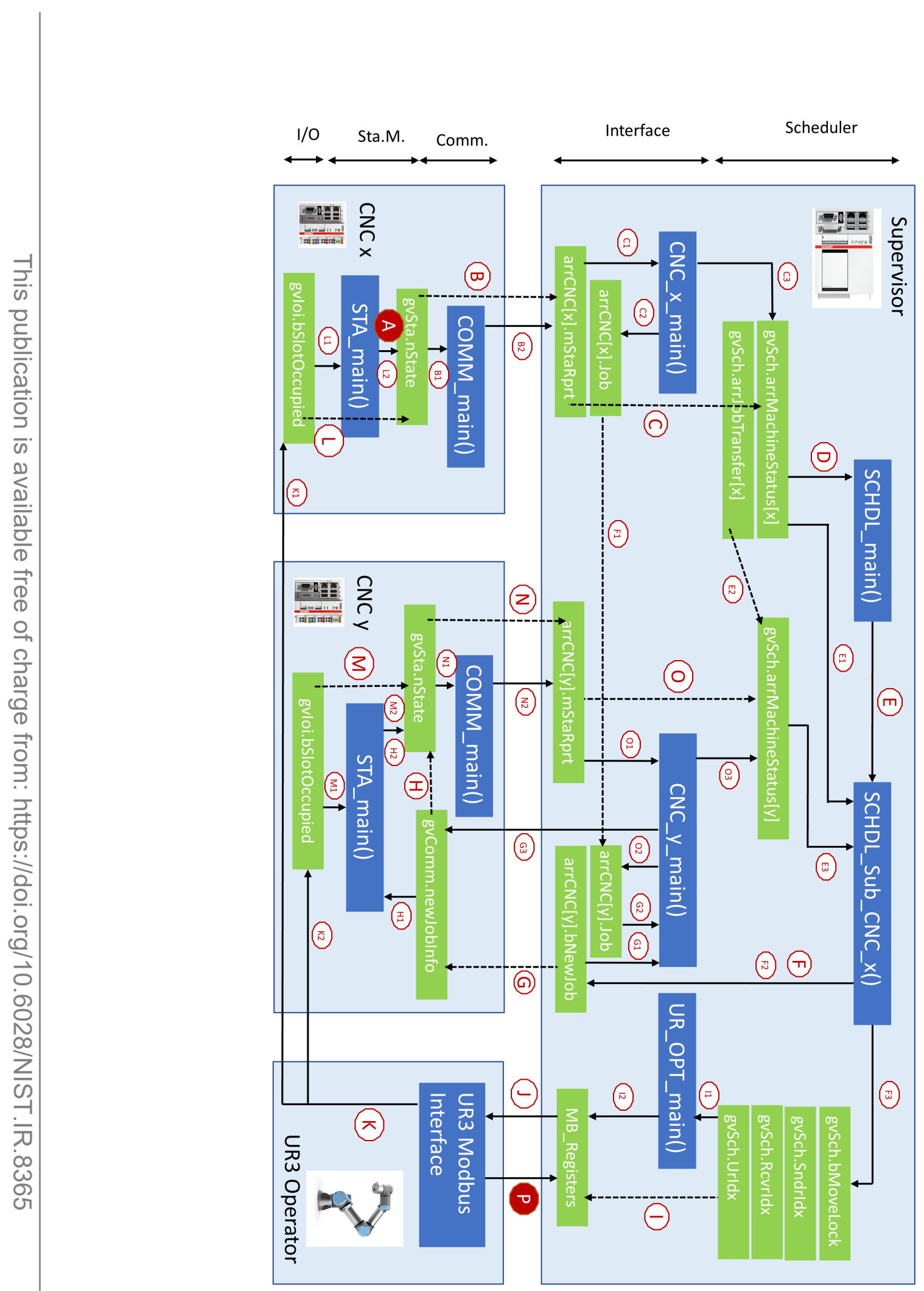

Fig. 16. Module interactions in a part transition case 
- [C1] CNC $\mathrm{x}$ interface at the supervisor loads the status report;

- [C2] CNC x interface updates the job status;

- [C3] CNC $x$ interface submits the status to the scheduler by writing into the machine status register;

- [D] The scheduler loads the CNC machine status to make scheduling decisions;

- [E] The scheduler assigns the job of moving the part from CNC $\mathrm{x}$ following the scheduling algorithm (which is independent from the architecture but includes at least the following information);

- [E1] The scheduler loads CNC x's status;

- [E2] The scheduler looks for the next hop station from $\mathrm{x}$ based on the job type;

- [E3] The scheduler loads the status at the next hop station, i.e., CNC y;

- [F] The scheduler makes the moving scheduling if CNC y is in the "IDLE/EMPTY" state;

- [F1] The job information is copied from CNC x's interface to CNC y's interface;

- [F2] The scheduler sets the new job notification to CNC y (followed by Step $\mathrm{G})$;

- [F3] The scheduler writes the scheduling information (followed by Step I);

- [G] The new job information is shared with the remote CNC y;

- [G1] The CNC y's interface at the supervisor detects a new job assigned by the scheduler;

- [G2] The CNC y's interface at the supervisor loads the job information;

- [G3] The CNC y's interface sends the new job information to CNC y via ADS;

- $[\mathbf{H}]$ The new job notification updates the machine's status at CNC y;

- [H1] CNC y's state machine detects the arrival of new job description;

- [H2] CNC y's state machine switch to "EXPECT NEW" waiting for the incoming part;

- [I] The Supervisor's scheduling decision is transformed into the Modbus commands for the UR3 operator;

- [I1] The Operator's interface at the supervisor detects the new assignment;

- [I2] The Operator's interface writes the scheduling information into the shared Modbus registers that are read/written by the Operator; 
- $[\mathbf{J}]$ The Operator obtains the assigned job through Modbus Register Read;

- [K] The Operator follows the job assignment to move the part from CNC $\mathrm{x}$ to CNC $\mathrm{y}$

- [K1] The Operator removes the part from CNC x (followed by Step L);

- [K2] The Operator places the part into CNC y (followed by Step M);

- [L] The part departs from CNC x and triggers the state transition at CNC x;

- [L1] The state machine at CNC $\mathrm{x}$ detects the departure of the part;

- [L2] The state machine at CNC x updates the state as "IDLE/EMPTY";

- $[\mathrm{M}]$ The part arrives at $\mathrm{CNC}$ y and triggers the state transition at $\mathrm{CNC}$ y;

- [M1] The state machine at CNC y detects the arrival of the part;

- [M2] The state machine at CNC y updates the state as "PART LOADED";

- [N] The new state at CNC y is reported to the supervisor via routine status report;

- [N1] The communication module at CNC y loads current state and formulates the report message;

- [N2] The communication module sends the report message to the supervisor via ADS Write;

- [O] The CNC y's interface at the supervisor updates the state of CNC y at the scheduler (similar as Step C);

- [01] The interface at the supervisor loads the newly received status report;

- [02] The interface writes the new state into the Job description;

- [03] The interface updates CNC y's state at the scheduler;

- [P (END)] The UR3 OPT returns to its Home position and notifies the supervisor.

Similarly, the main interactions between work-cell (sub-)modules in a part inspection scenario are listed as follow,

- [A (START)] A part being processed at CNC x requests the inspection;

- [B] COMM of CNC x sends an inspection request via ADS;

- [C] CNC_x_interface at the supervisor detects the new inspection request;

- [D] CNC $\mathrm{x}$ interface at the supervisor sets the flag $(\mathrm{ON})$ in the Inspection Request variable; 


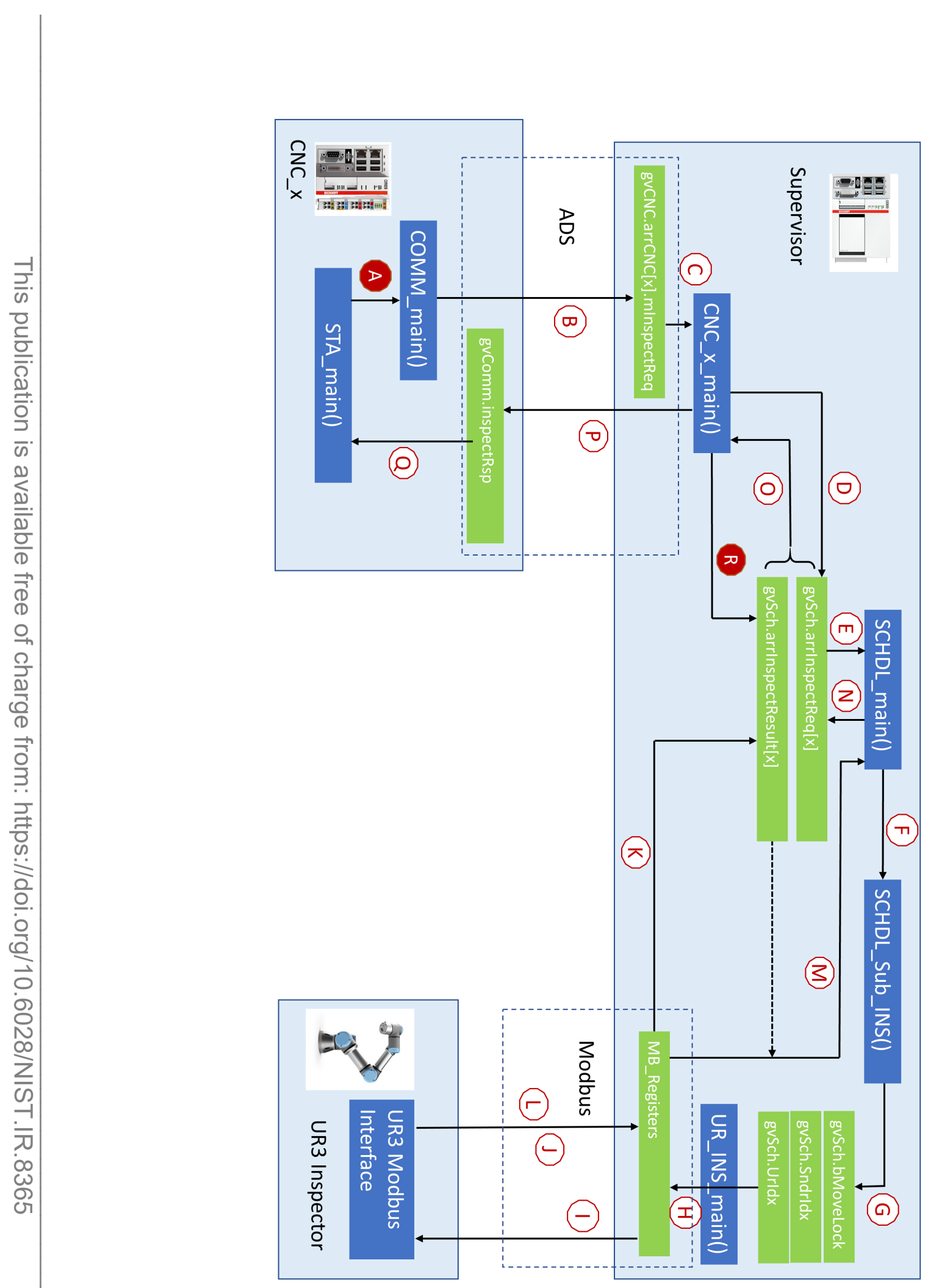

Fig. 17. Module interactions in a part inspection case 
- [E] The scheduler of the supervisor collects CNC inspection requests;

- [F] The scheduler picks the inspection CNC target by calling SCHDL_Sub_INS;

- [G] SCHDL_Sub_INS assigns an inspection task to the UR3 INS robot;

- $[\mathbf{H}]$ The INS interface at the supervisor writes the task into Modbus registers;

- [I] INS retrieves commands via Modbus and executes them;

- [J] INS writes inspection results back into Modbus registers;

- $[\mathbf{K}]$ The inspection result is updated from Modbus registers;

- [L] INS finishes the task, returns its Home position, and notifies the supervisor;

- $[\mathbf{M}]$ The scheduler at the supervisor waits until both Step K and L are completed;

- $[\mathbf{N}]$ The scheduler at the supervisor removes the flag in the Inspection Request (OFF) and waits for the next call;

- [O] CNC_x_interface at the supervisor waits until Step K and N are done;

- [P] CNC_x_interface at the supervisor sends an inspection response via ADS back to $\mathrm{CNC}$;

- [Q] CNC x processes the received Inspection Response;

- [R (END)] CNC_x interface at the supervisor clears the finished result for the next call.

\section{Network Components}

To fulfill the coordination between work-cell modules, a communication network is deployed in the testbed that links distributed modules together and enables communications with multiple purposes. Besides process variable updates and control commands transmissions in the emulated production, the network also enables data transmissions for the other testbed functions, such as distributed clock synchronization and measurement data collection. Therefore, the testbed network needs to carry multiple heterogeneous traffic streams. In this section, we focus on explaining network components implemented in the testbed to serve these applications. First, communication traffic needs are identified in the links; then, the network architecture comprised of wired Ethernet links is illustrated; finally, the extension to wireless links is discussed. 


\subsection{Communications in Work-Cell Applications}

As discussed earlier in Section 4 and illustrated in Fig. 15, communications in the work-cell is centered around the supervisor, which manages the production process and coordinates various modules. In the network topology, the supervisor also acts as the information hub and gateway for operational data flows, both internal and external ones. Messages within and beyond the work-cell are formalized by different communication protocols according to individual applications including, but not limited to, order handling, part status tracking, machine diagnostics, and safety alerts. We will first examine these communication messages and their requirements on the links.

\subsubsection{Remote HMI Operations}

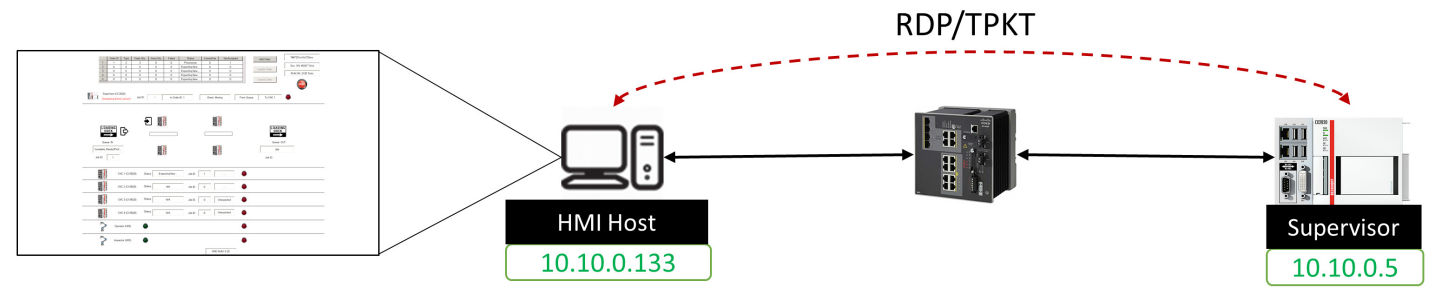

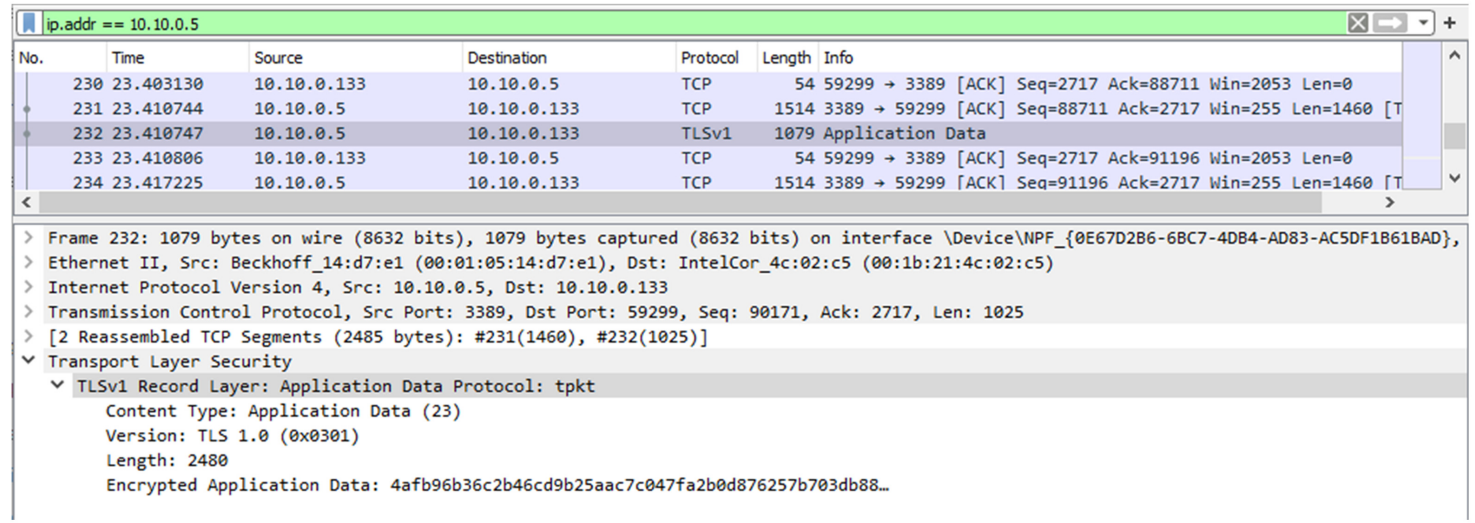

Fig. 18. Communications for remote HMI operations and packet samples captured by WireShark

The main configuration and monitoring tasks in the testbed are through the HMI. When the work-cell HMI is operated remotely, a Windows remote desktop connection is established between the host Windows machine and the supervisor PLC as shown in Fig. 18. ${ }^{2}$ Windows manages such a connection using a proprietary application protocol for Terminal Server services, which is known as Remote Desktop Protocol (RDP). RDP uses the ISO Transport Service on top of the TCP (TPKT) protocol in the transport layer. TPKT uses the particular TCP port 3389 for RDP applications. The RDP traffic presents an on-demand

\footnotetext{
${ }^{2}$ Messages are captured at a test access point (TAP) device in the Ethernet link and recorded by WireShark. The basic structure of Ethernet packets is presented in Appendix A.
} 
transmission pattern, i.e., RDP messages are transmitted in the link only when the HMI content needs to be updated in the host's remote desktop application. For example, if the remote desktop in the host is minimized, no RDP messages are transmitted in this case. The traffic load also largely depends on dynamics of the displayed desktop content, such as how fast it changes and the proportion of dynamic areas compared to the whole view. In the testbed, the active HMI session exhibits an intermittent and light traffic pattern with an average throughput less than $100 \mathrm{kbps}^{3}$

\subsubsection{Supervisor-CNC Machines Interactions}

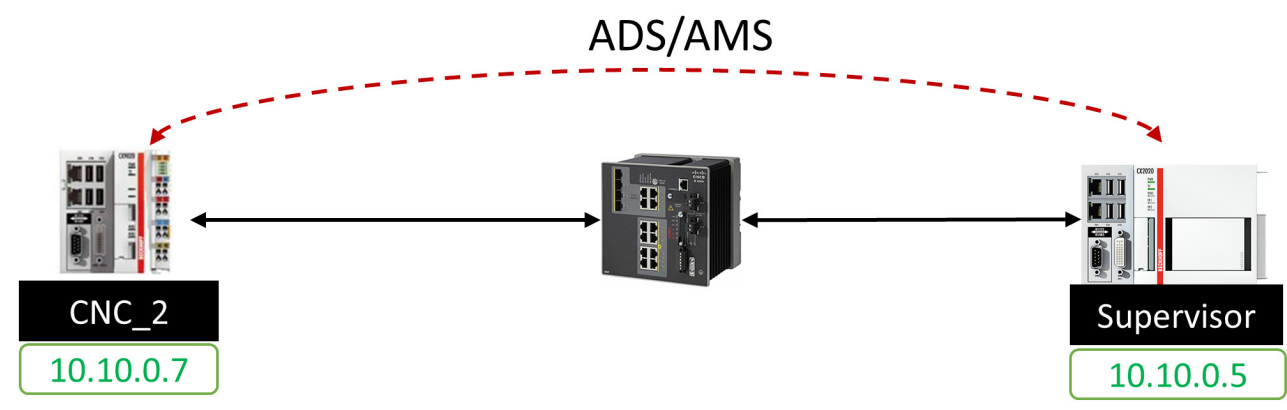

\begin{tabular}{|c|c|c|c|c|c|c|}
\hline No. & & Time & Source & Destination & Protocol & Length Info \\
\hline$\Varangle$ & 15 & 0.101938518 & 10.10 .0 .5 & 10.10 .0 .7 & AMS & 104 AMS Request \\
\hline & 16 & 0.119980566 & 10.10 .0 .7 & 10.10 .0 .5 & AMS & 112 AMS Request \\
\hline & 17 & 0.121955356 & 10.10 .0 .5 & $10.10 \cdot 0.7$ & AMS & 96 AMS Request \\
\hline$<$ & 10 & A A anmagara & in in n & in in $n$ & nus & And ane n n \\
\hline
\end{tabular}

Frame 16: 112 bytes on wire ( 896 bits), 112 bytes captured ( 896 bits) on interface enp6s0f1, id 0

Ethernet II, Src: Beckhoff_37:f9:c7 (00:01:05:37:f9:c7), Dst: MoxaTech_66:a8:63 (00:90:e8:66:a8:63)

Internet Protocol Version $\overline{4}$, Src: 10.10 .0 .7 , Dst: 10.10 .0 .5

> Transmission Control Protocol, Src Port: 49155, Dst Port: 48898, Seq: 265, Ack: 185, Len: 58

$\checkmark$ AMS

AMS Target Net Id: 5.20.215.224.1.1

AMS Target port: 851

AMS Sender Net Id: 5.55.249.199.1.1

AMS Sender port: 350

CmdId: ADS Write (3)

$>$ Stateflags: 0x0004

cbData: 20

ErrorCode: NO ERROR (0x00000000)

InvokeId: 0xff380015

$\checkmark$ ADS Write Request

IndexGroup: 0x0000f005

IndexOffset: $0 \times 788002 c 3$

CbLength: 8

Data

Fig. 19. The supervisor-CNC Communications and packet samples captured by WireShark

${ }^{3}$ The throughput was calculated during normal operations when the remote HMI main view stayed on the host desktop and was shown in full screen . In some cases, e.g., when the remote desktop switches between different HMI views or between HMI and the other Windows views, it may cause a larger instant RDP traffic up to $1 \mathrm{Mbps}$. 
In the coordination process as discussed in Section 4.5, the supervisor needs to maintain continuous communications with $\mathrm{CNC}$ machines for tracking production efficiency and device health. Various message types are identified in multiple concurrent active sessions in the links. First, the supervisor routinely receives the status update from the CNC machine so that it can estimate the load at each single job stop, which is reflected in its scheduling decision. Second, the supervisor also routinely updates the work-cell status to field machines so that individual production modules have the real-time status of their partners that are used to coordinate their action. Furthermore, there are state-based on-demand communications, such as the inspection request-response conversions in the links.

In the testbed, the data exchanged between the supervisor (i.e., a Beckhoff PLC) and CNC machine controllers (i.e., also Beckhoff PLCs) are formatted as Automation Device Specification (ADS) commands, which are defined in the proprietary communication protocol for Beckhoff's TwinCAT devices known as Automation Message Specification (AMS). ADS/AMS is a medium-independent protocol. As shown in Fig. 19, ADS/AMS messages are carried in transmission control protocol/Internet protocol (TCP/IP) packets. The PLC runtime uses the reserved TCP port 851 as the identifier. Details about ADS/AMS are presented in Appendix A. The PLC uses the EtherCAT protocol to manage internal data flows with its terminal modules, such as motion control and I/O modules, which is outside the scope of this testbed design.

\subsubsection{Supervisor-Robots Interactions}

Similar to communications in the supervisor-CNC link, the supervisor also exchanges information with the UR3 robots for status update and job instructions, as shown in Fig. 20. Modbus is used in the links from the supervisor to UR3 control boxes of OPT and INS, respectively. Modbus allows data exchange between heterogeneous industrial appliances through the shared registers. In Modbus link, the supervisor serves as the Modbus server, which maintains the shared data in its memory. Through Modbus queries, the client can visit the remote data at particular register addresses. In the testbed, UR3 robots are the clients who send the write/read commands to the supervisor for updating robot status and obtaining instructions for the following moves. Modbus messages are identified by the TCP port 502. Basic information of Modbus communications is also provided in Appendix A.

\subsubsection{Robotic Subsystem Communications}

Robots are usually equipped with peripherals to better perform in different tasks. In this testbed, every UR3 robot has one gripper attached to its end effector along with a F/T sensor. The UR3 control box communicates with peripherals through TCP/IP socket communications. In the testbed, the F/T compute box is connected to the UR3 controller in the same subsystem switch as shown in Fig. 21. Every $8 \mathrm{~ms}$, the controller updates the compute box once with UR3 system status which is formatted in a TCP package containing 139 values in a 1108 byte payload. TCP messages are sent through the UR3's TCP port for 


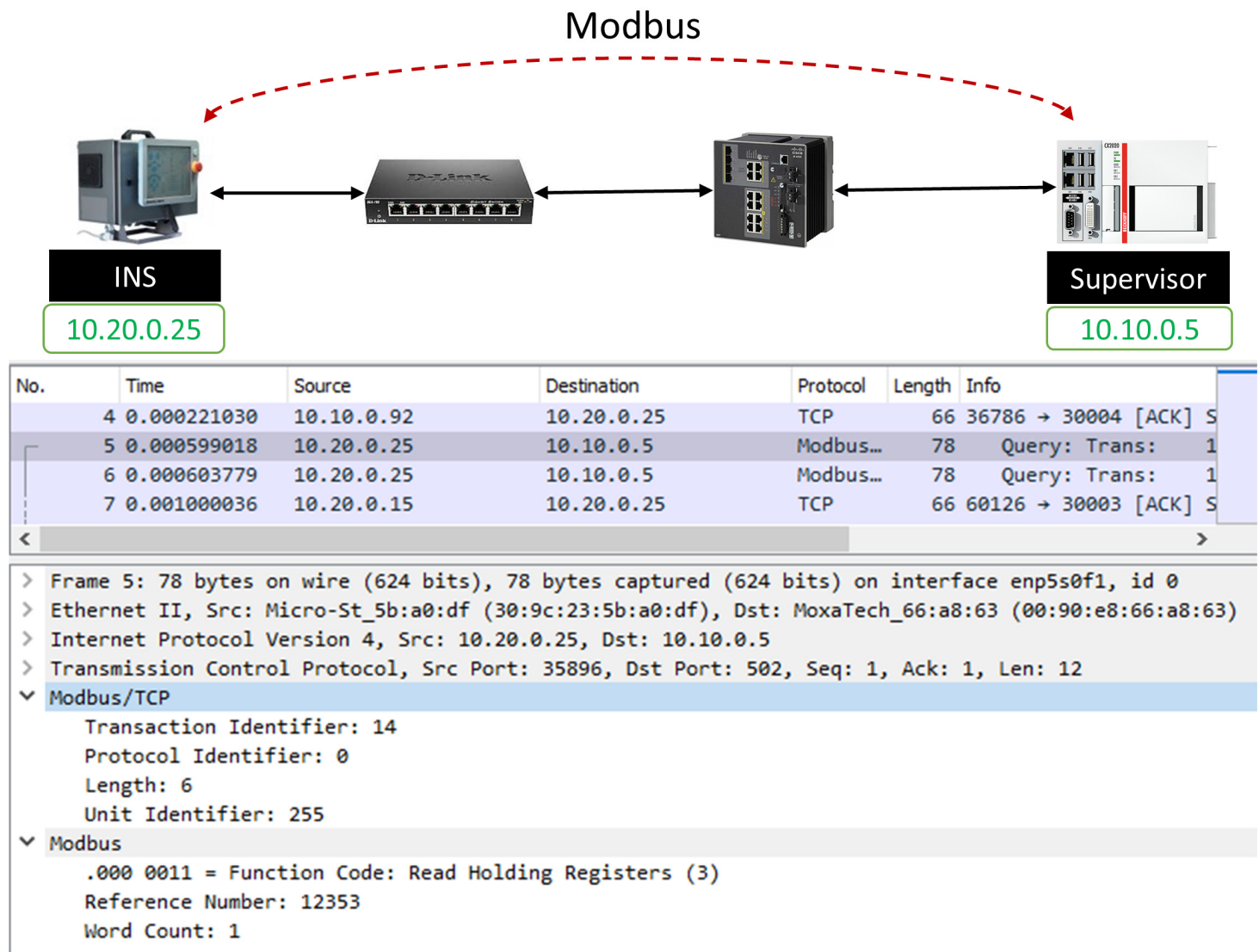

Fig. 20. The supervisor-robot communications and packet samples captured by WireShark

real-time traffic (30003). The F/T compute box software also updates the controller with the real-time $\mathrm{F} / \mathrm{T}$ sensor values at $125 \mathrm{~Hz} .^{4}$

\subsubsection{A Summary of Communication Traffic}

Table 2 summarizes the emulated data flows in the testbed.

\subsection{Networking Architecture}

In support of work-cell communications, the testbed first implements fully wired connections between production modules. Ethernet enables inter-node communications in a wired medium. First, each production module in our testbed has one or more on board RJ-45 slots along with built-in Ethernet-based local area network (LAN) adapters (see Appendix D for network interfaces of individual devices). Second, the identified communication messages

${ }^{4}$ Current F/T firmware only supports an error-free connection, such as in an Ethernet link, with the control box. Missed controller updates will result in the link disconnection, which calls a system exception and pauses the robot operation. It requires a manual reset of the compute box. 


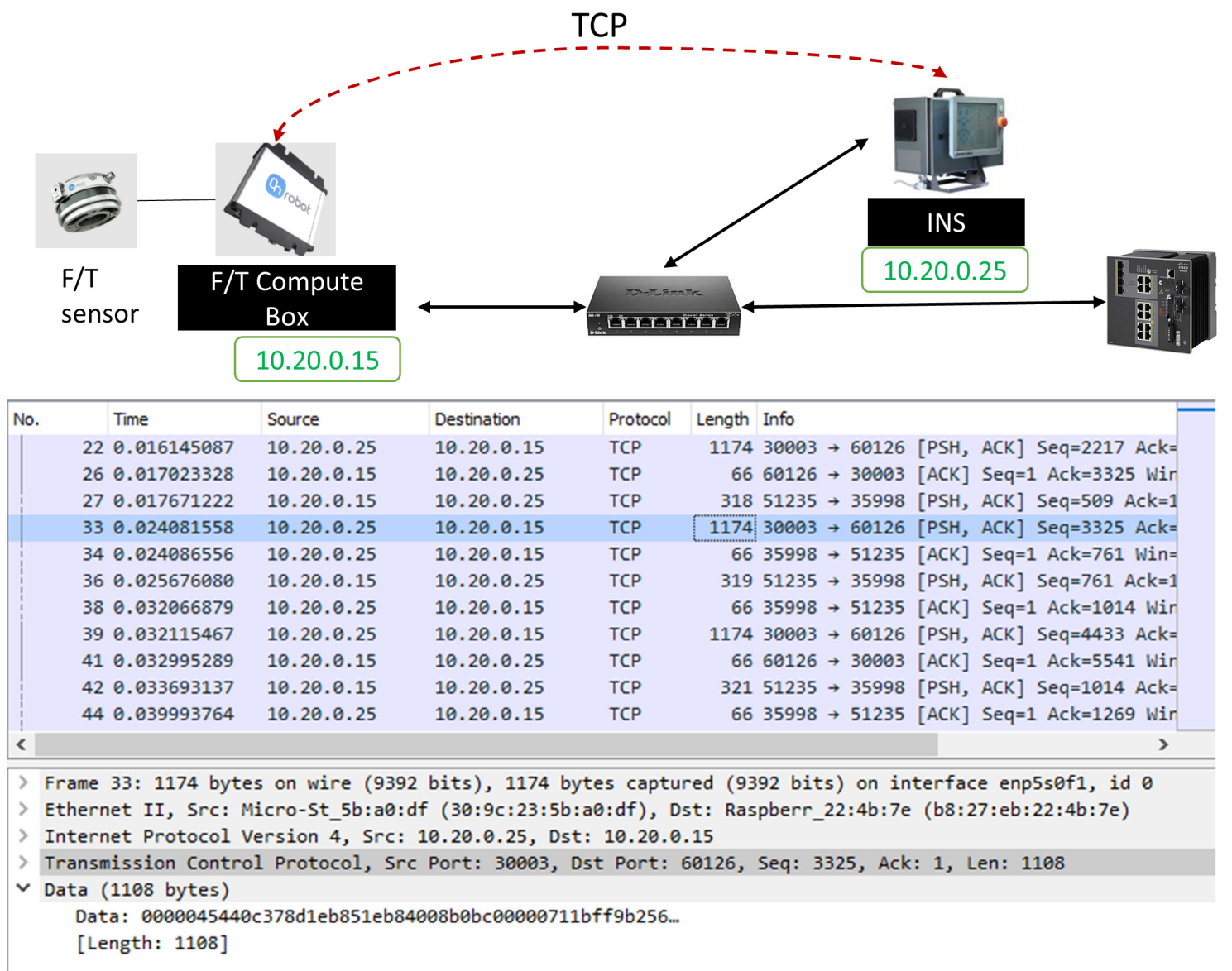

Fig. 21. Communications between the robot controller and the F/T sensor with packet samples captured by WireShark

in Section 5.1 are originally designed for the carrier frame structure in terms of TCP/IPEthernet packets (see Appendix A for packet frame structures).

Fig. 22 illustrates the wired networking architecture. The network backbone is formed by a bundle of two Cisco IE-4000 industrial Ethernet switches. Each IE-4000 switch has 12 gigabit Ethernet ports that provide managed Layer 2 switching functions as well as advanced features such as the support of time synchronization (e.g., NTP and PTPv2) and time sensitive networking (TSN). One gigabit port in each switch is reserved for bridging switches in trunk mode. Therefore, there are in total 22 gigabit ports available for work-cell communications and the other testbed communication functions.

All production modules, which are also testbed network nodes, use one of their onboard Ethernet ports for work-cell production communications. The supervisor PLC and CNC PLCs are directly plugged into the Cisco switch bundle through Ethernet cables. The UR3 robots have their own sub-network switches to interconnect parts in each robotic subsystem. Two D-Link unmanaged switches are deployed for robot sub-networks, one for 
Table 2. Specifications of sampled data flows between work-cell components

\begin{tabular}{|l|l|l|l|l|}
\hline \multirow{2}{*}{ Link } & Data & Update Rate & $\begin{array}{l}\text { Size } \\
\text { (Bytes) }\end{array}$ & Protocol \\
\hline \multirow{2}{*}{$\begin{array}{l}\text { Supervisor } \\
\text {-CNC }\end{array}$} & Status report & $1 \mathrm{~Hz}-100 \mathrm{~Hz}$ & $10 \mathrm{~s}$ & ADS \\
\cline { 2 - 5 } & Safety & $100+\mathrm{Hz}$ & $10 \mathrm{~s}$ & ADS \\
\cline { 2 - 5 } & $\begin{array}{l}\text { Inspection } \\
\text { request/response }\end{array}$ & On-demand & $10 \mathrm{~s}$ & ADS \\
\hline $\begin{array}{l}\text { PLC } \\
\text {-Peripheral }\end{array}$ & Motion control & $1000 \mathrm{~Hz}$ & A few & ADS \\
\hline $\begin{array}{l}\text { Supervisor } \\
\text {-Robot }\end{array}$ & Actuation & $1 \mathrm{~Hz}-50 \mathrm{~Hz}$ & A few & Modbus \\
\cline { 2 - 5 } $\begin{array}{l}\text { Robot } \\
\text {-Peripheral }\end{array}$ & Safety & $125 \mathrm{~Hz}$ & A few & Modbus \\
\hline \multirow{2}{*}{$\begin{array}{l}\text { Supervisor } \\
\text {-External }\end{array}$} & HMI & $100 \mathrm{~Hz}$ & $\begin{array}{l}100 \mathrm{~s} \\
-1000\end{array}$ & TCP/IP \\
\cline { 2 - 5 } & IoT & $10 \mathrm{~Hz}-50 \mathrm{~Hz}$ & $\begin{array}{l}100 \mathrm{~s} \\
-1000 \mathrm{~s}\end{array}$ & ADS \\
\hline
\end{tabular}

* Note: The size is referred to as the application data size.

each. Each D-Link switch has 8 gigabit Ethernet ports and is linked with the backbone.

The testbed uses IPv4 addresses to manage communication devices. The gateway is 10.10.0.1 and the subnet mask is 255.0.0.0. For individual appliances that have direct connections to the backbone, they are assigned with IP addresses in 10.10.0.x. For robot sub-networks, INS subsystem has IP addresses in 10.20.0.x while OPT components have IP addresses in 10.30.0.x. Specific IP addresses are illustrated in the network diagrams of Appendix E.

Additional network connections are also planned in the testbed that provide measurement data links and other complimentary features, such as time synchronization services. These supportive connections will be introduced in the measurement framework of Section 6.

\subsection{Wireless Extension}

Wireless links are added into the testbed network to verify the capability of wireless technologies in support of mission-critical industrial communications. Upon the first release of this report, WLAN connections have been implemented in the testbed where one or more selected Ethernet connections are replaced. Since industrial appliances are originally designed for Ethernet networks, there is no built-in wireless adapter available. We introduce Intel's Next Unit of Computing (NUC) devices as Ethernet-WLAN adapters that trans- 


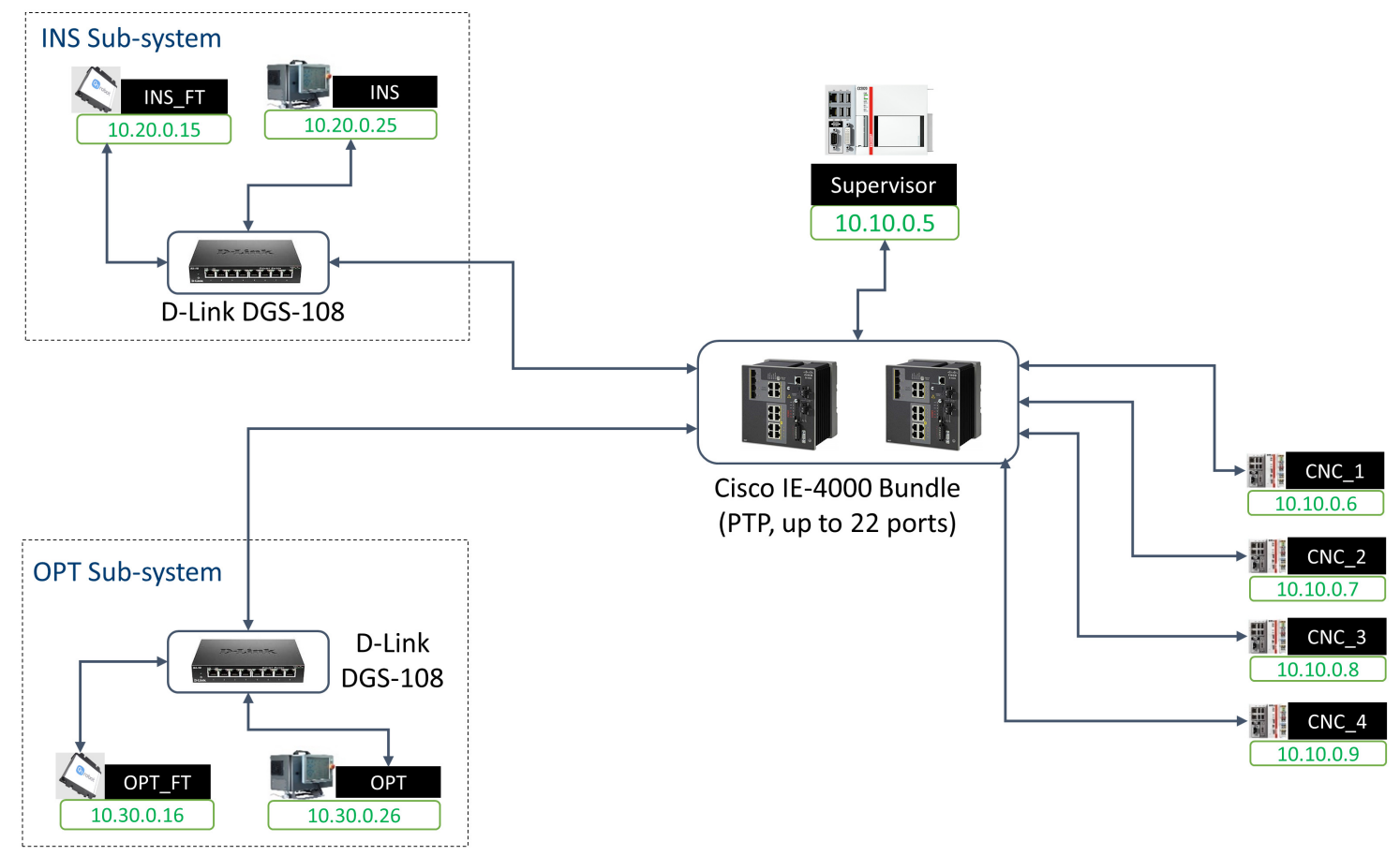

Fig. 22. Work-cell network architecture using full wired Ethernet connections

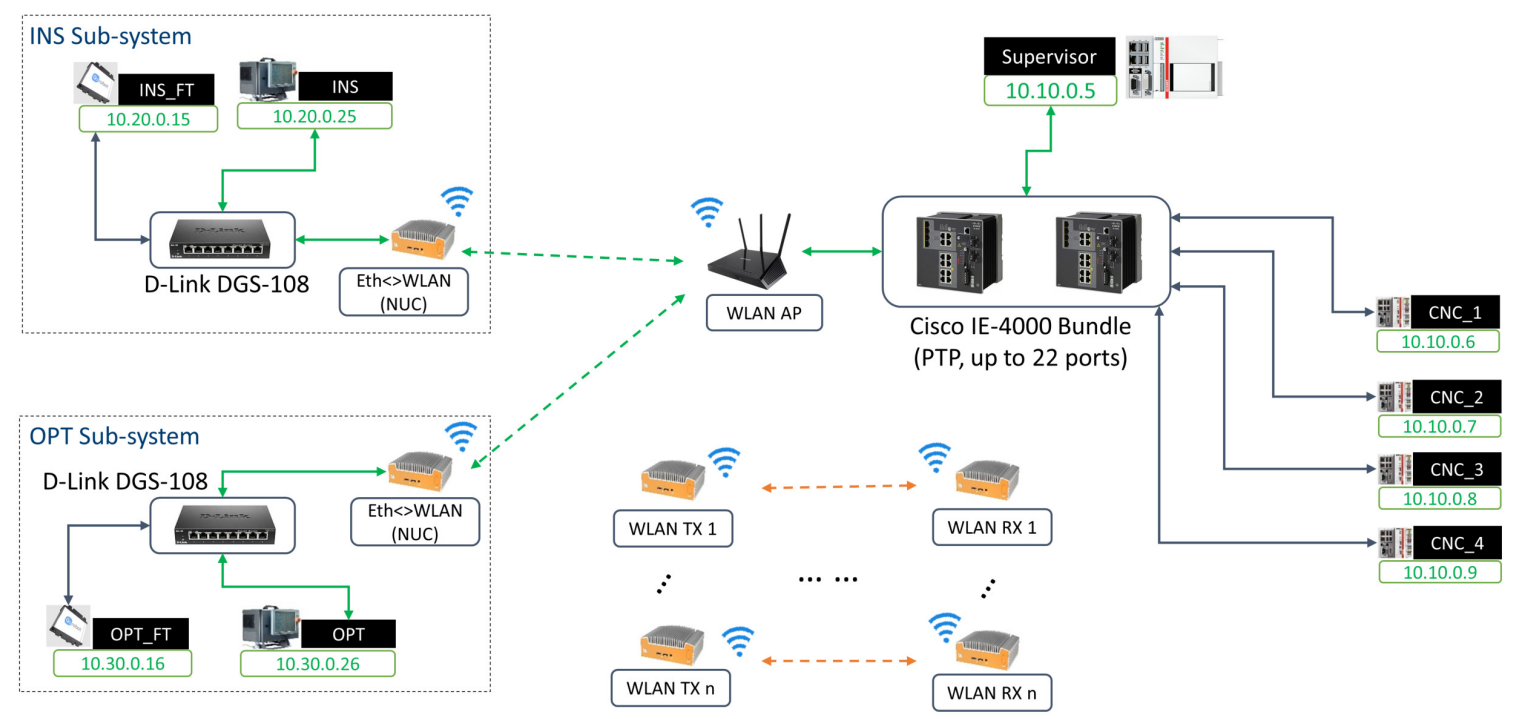

Fig. 23. Wireless extension to the work-cell network architecture

late industrial messages between Ethernet frames and WLAN packets. Fig. 23 illustrates the introduced wireless links in the work-cell network. In this use case, UR3 subsystems communicate with the rest of work-cell through WLAN links. Specifically, each UR3 subnetwork is equipped with an Intel NUC serving as the Ethernet-WLAN adapter. A Netgear 
AC1900 WLAN router (R7000) is used as the WLAN access point (AP) and connected to the switch bundle. WLAN connections between NUCs and the AP enable UR3 controllers and the supervisor to coordinate the OPT and INS operations, respectively. Wireless transmissions comply with IEEE $802.11 \mathrm{~b} / \mathrm{g} / \mathrm{n}$ protocols. WLAN radio modules negotiate to determine the working mode based on the instant channel quality. One or more background traffic generators can also be deployed in the working WLAN channels that emulate the interference in real industrial sites. These traffic generators are performed by extra pairs of Intel NUCs in the testbed that run iPerf scripts to create user datagram protocol (UDP) unicast packets as background stress data.

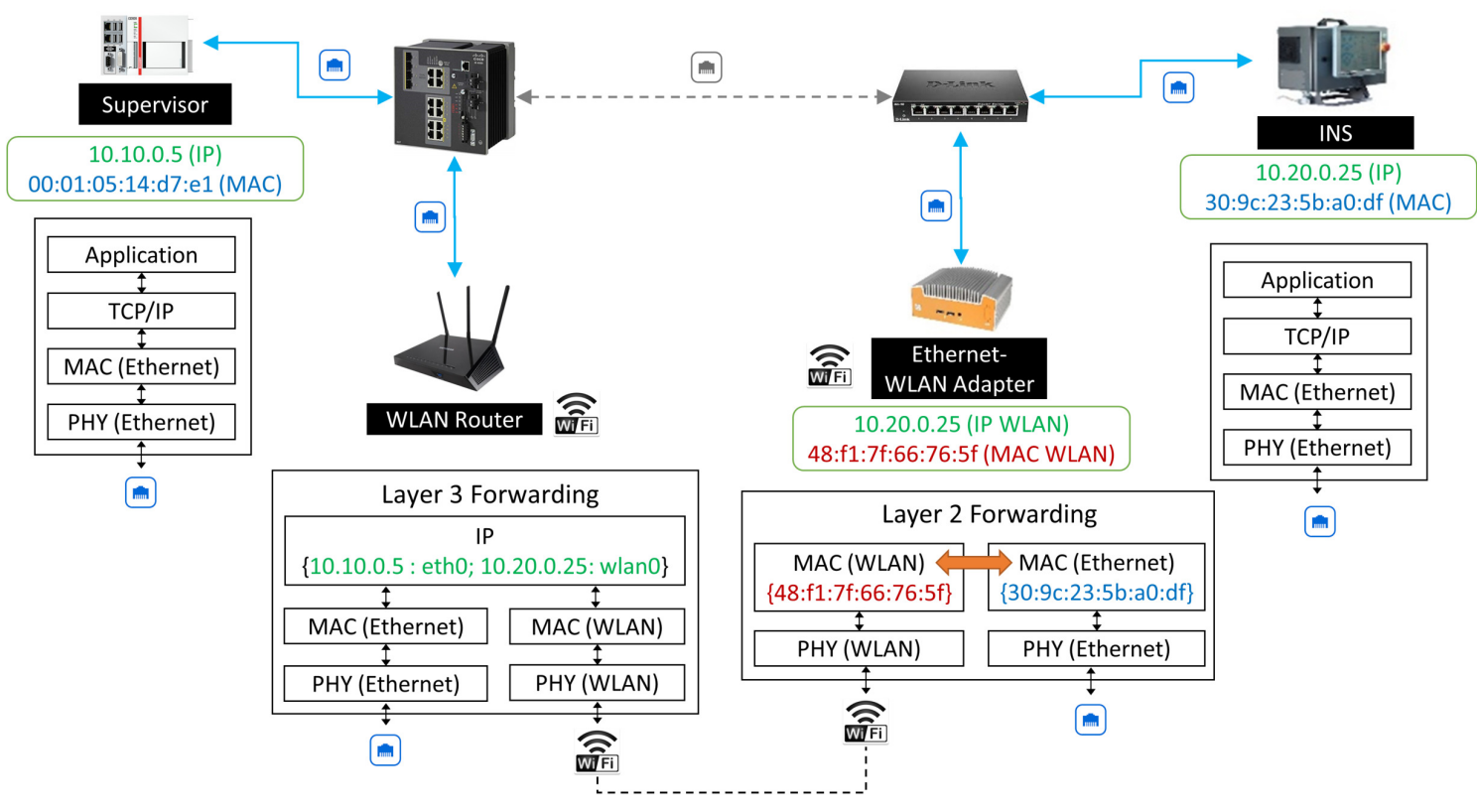

Fig. 24. Illustration of Layer 2 forwarding through the Ethernet-WLAN adapter

NUC by its nature is a barebone computer running Linux operating system (OS), e.g., Ubuntu 14.04 used in the testbed. Equipped with network interfaces including Ethernet and IEEE $802.11 \mathrm{~b} / \mathrm{g} / \mathrm{n}$ adapters, NUCs can be programmed to work in different modes to meet the service requirements of different roles in the testbed. Generally, NUCs are designed to work in the following roles: Ethernet-WLAN adapter, wireless sniffer, and background traffic generator/sink.

When working as an Ethernet-WLAN adapter, it converts the received Ethernet frames to WLAN packets, and vice versa, which is called the Layer 2 forwarding. Compared to the Layer 3 forwarding at a router which handles IP packets, the NUC first resumes the in-bound medium access control (MAC) protocol data unit (PDU) (i.e., the IP packet(s) encapsulated in a MAC frame), repacks it into another MAC frame format, and sends in the new medium as the out-bound data. Fig. 24 illustrates how the NUC serves as the UR3 inspector's Ethernet-WLAN adapter in its communication with the supervisor. 
Since such conversion is performed by a NUC program in software, it is a type of "soft" forwarding approach. The entire forwarding process is transparent to end users as the endto-end connection is IP-based, whose information is intact in the lower layer process. The only change in the network is that the NUC adapter is treated as an alias of the served host along the path. In the case as shown in Fig. 24, all en route devices (i.e., the supervisor, the Cisco switch bundle, and the WLAN router) address the NUC as the counterpart to the supervisor in supervisor-INS communications. For instance, the supervisor uses the NUC's MAC address, not the INS's one, in the MAC destination field of a message destined for INS. Accordingly, the NUC needs to replace it by the INS's MAC address in the forwarded packet.

As a wireless sniffer, the NUC uses the iw command, i.e., a new command-line interface (CLI) configuration utility for wireless devices, to create a virtual interface, associate it to the physical WLAN interface, and set it in the monitor mode. Once specifying the working WLAN channel for the virtual interface, we can run TShark or Tcpdump to sniff all WLAN packets in the channel.

In experiment scenarios considering concurrent WLAN transmissions as the co-channel interference, extra NUCs can be paired up as background traffic sources and sinks using Iperf, an Internet network test tool, to generate managed background traffic [23].

\section{Measurement Framework}

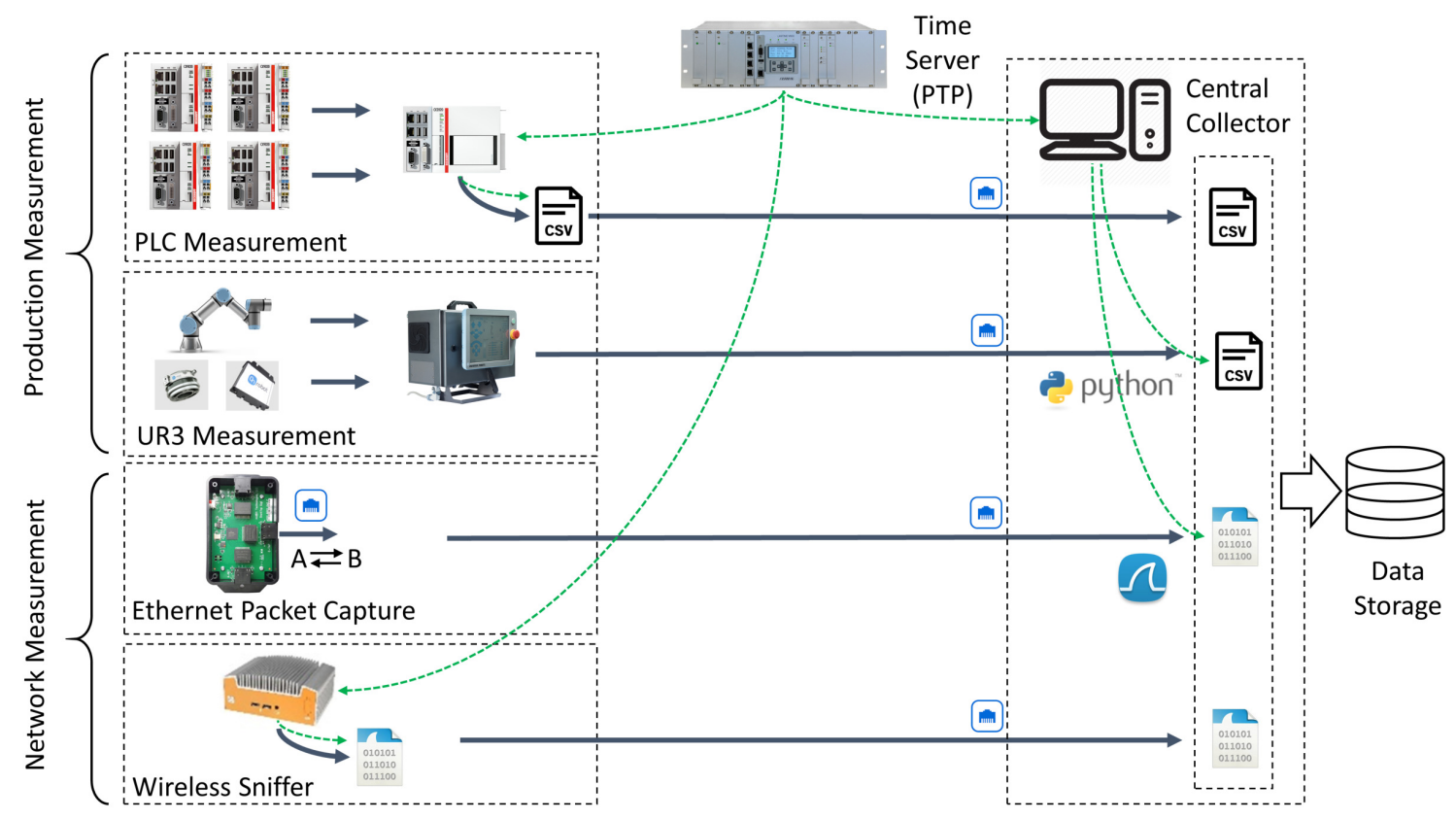

Fig. 25. The testbed measurement framework

Measurements are taken in various points of interest to obtain data showing features of 
the emulated production processes and network operations as well as their relationships. We have introduced types of measurement apparatus in the testbed and developed a centralized framework parallel to work-cell operations. As shown in Fig. 25, the developed measurement framework provides complimentary functions and features that enhance data collection in the testbed and feed further analysis with trustworthy data. In this section, we will systematically review individual measurement components. Specifically, data types in measurements are enumerated and their properties are explained; tools implemented in the framework for obtaining corresponding measurement data are introduced whose collection flows are shown as blue arrows in Fig. 25; time synchronization over measurement probes is also discussed that unifies timed observations from distributed data records, which is presented as green dashed arrows in the figure.

\subsection{Measurement Data Collection}

We have identified data classes by their sources, i.e., production modules and network components, which are labeled as operational and network-related, respectively. Accordingly, measurement tools and collection procedures are developed for individual data sets.

Testbed data are managed in the central collector which is a desktop computer running Ubuntu 18.04 and equipped with one 1 terabyte (TB) solid-state drive (SSD) for data storage, as shown in Fig. 25. The collector has ten gigabit Ethernet ports that can be connected to testbed apparatus for data collection in the work-cell. Details about the full network diagram are illustrated in Appendix E. Local measurement results, such as the ones collected at the supervisor PLC and wireless sniffer, are transferred to the collector after individual experiments. Meanwhile, real-time measurement data, such as the UR3 real-time data exchange (RTDE) data and network packet captures, are directly recorded at the central collector who assigns independent measurement links and runtime threads for individual collection programs. Data is saved with timestamps for post data analysis, and data files are indexed with the unique name regarding each experiment.

\subsubsection{Operational Data Collection}

Operational data refer to the recorded process variables and control commands saved during the operation of production modules. Data from the supervisor, CNC machines, and UR3 robots belong to this class. For individual modules, state-related variables are the primary data source, which contains rich behavioral information for modeling the digital copy of state machines and analyzing the operation performance in different situations.

As discussed in Section 4, the supervisor watches over the entire work-cell's production. Therefore, the first measurement point is set in the supervisor as shown in Fig. 25. Data from the supervisor indicate how the scheduler manipulates individual machinery operations based on its local view of work-cell status. All GVL variables used by PLC programs can be routinely reported to the collector as measurement data. Currently, we focus on testbed data that may affect the scheduler's decision and coordination performance. 
Since status information is reported in a routine manner through the network, network connections may affect the supervisor's response to any state change in the production process, which is the main analysis goal in this work.

Optional data at individual CNC machines and robots can also be subscribed that reflect the local view of machine status. By comparing the initiation time of a production command and its real actuation time, it implies the control delay which consists of the processing time as well as the delay in communication links, which provides the insight of network impact on production performance. For CNC machines, since emulated CNC controllers are also implemented in Beckhoff's PLCs which share the same program architecture as the supervisor PLC, their GVL variables can be subscribed in measurements. Details of measuring PLC data are explained in Appendix B.1. PLC data are recorded at $125 \mathrm{~Hz}$ and stored locally in the PLC's hard drive. Each round of experiment data is saved in a single CSV file.

For UR3 robots, internal program variables and URCaps' information are accessible through the UR's RTDE interface. RTDE outputs a comprehensive list of robot status variables such as position, velocity, acceleration, current, voltage, and other critical metrics. For example, in trajectory planning, both actual and target setpoints of position variables are collectible. Since RTDE data showcases internal operation status within robot subsystems, which are closely related with collaborative robot applications, such as work-cell safety, we set another set of measurement points at UR3s. RTDE measurement is configurable through an extensible markup language (XML) file where it is flexible to subscribe or remove variables in the measurement output. We refer the interested readers to check the supported robot state parameters in the RTDE guide [24]. RTDE provides periodic updates at $125 \mathrm{~Hz}$ through the TCP port 30004. The central collector retrieves real-time RTDE readings through TCP socket connections with control boxes of OPT and INS, respectively. Python scripts are developed here for RTDE data collection outputting results in CSV files.

\subsubsection{Network Traffic Captures}

Network traffic data are communication messages that are captured in links between workcell appliances. Data types have been identified in the earlier Section 5.1. In the testbed, we use network test access point (TAP) devices to passively capture Ethernet messages transmitted in the monitored links. As shown in Fig. 26, a simple TAP is usually equipped with three Ethernet ports. Port A and B are internally bridged, which enables through traffic to pass so that the monitored link does not break. During packet forwarding between $A$ and $\mathrm{B}$, the TAP makes one copy of each through packet and send it to the third port $(\mathrm{A} \rightleftarrows \mathrm{B})$ which is connected to a data collector. If the TAP has more than one $\mathrm{A} \rightleftarrows \mathrm{B}$ port on board, it generates multiple copies of through traffic, one for each.

Fig. 27 indicates locations of TAP devices that we have employed to monitor work-cell links carrying critical production messages. The central collector (not shown in this figure) reaches out to each deployed TAP device through a direct gigabit Ethernet link so that 

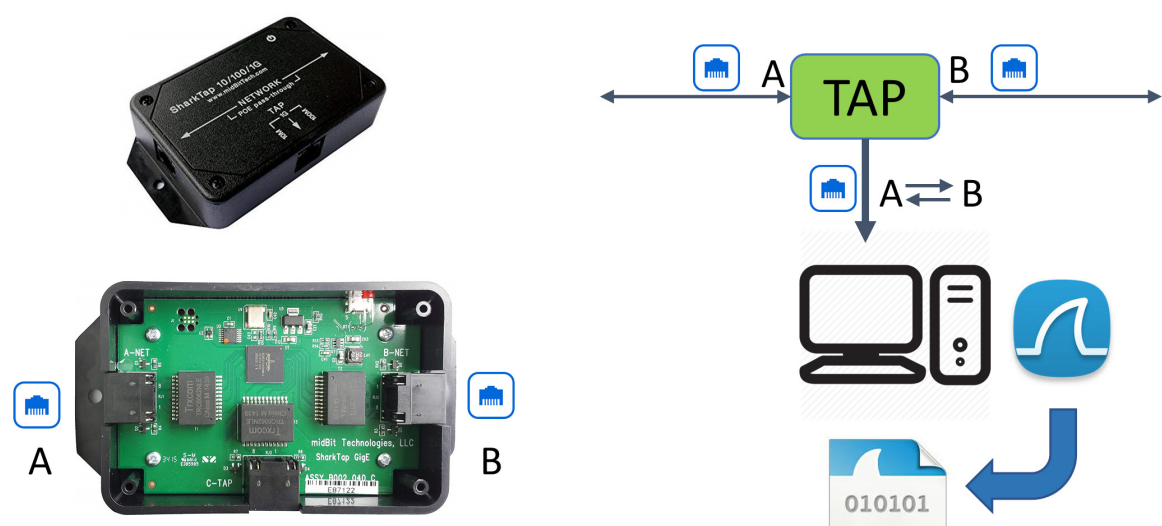

$A \rightleftarrows B$

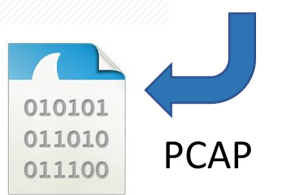

Fig. 26. Network traffic test access point (TAP) device



Fig. 27. Network traffic probes in the testbed network

the real-time traffic collection does not affect network load of work-cell communications or introduce bandwidth competition between TAP links. We refer the interested readers to check more details of link connections in the full network diagram of Appendix E. The collector collects link data by using the network protocol analyzer, e.g., TShark/WireShark. Each TAP link is monitored by an independent TShark thread whose data are saved in a separate packet capture (PCAP) file.

TAP devices only serve to capture Ethernet packets in wired connections. To further obtain wireless transmission quality information, we have also employed wireless sniffing tools in the measurement. Fig. 27 illustrates a wireless data collection case where an Intel NUC is deployed as a wireless sniffer co-located with the AP. Working in the monitor 


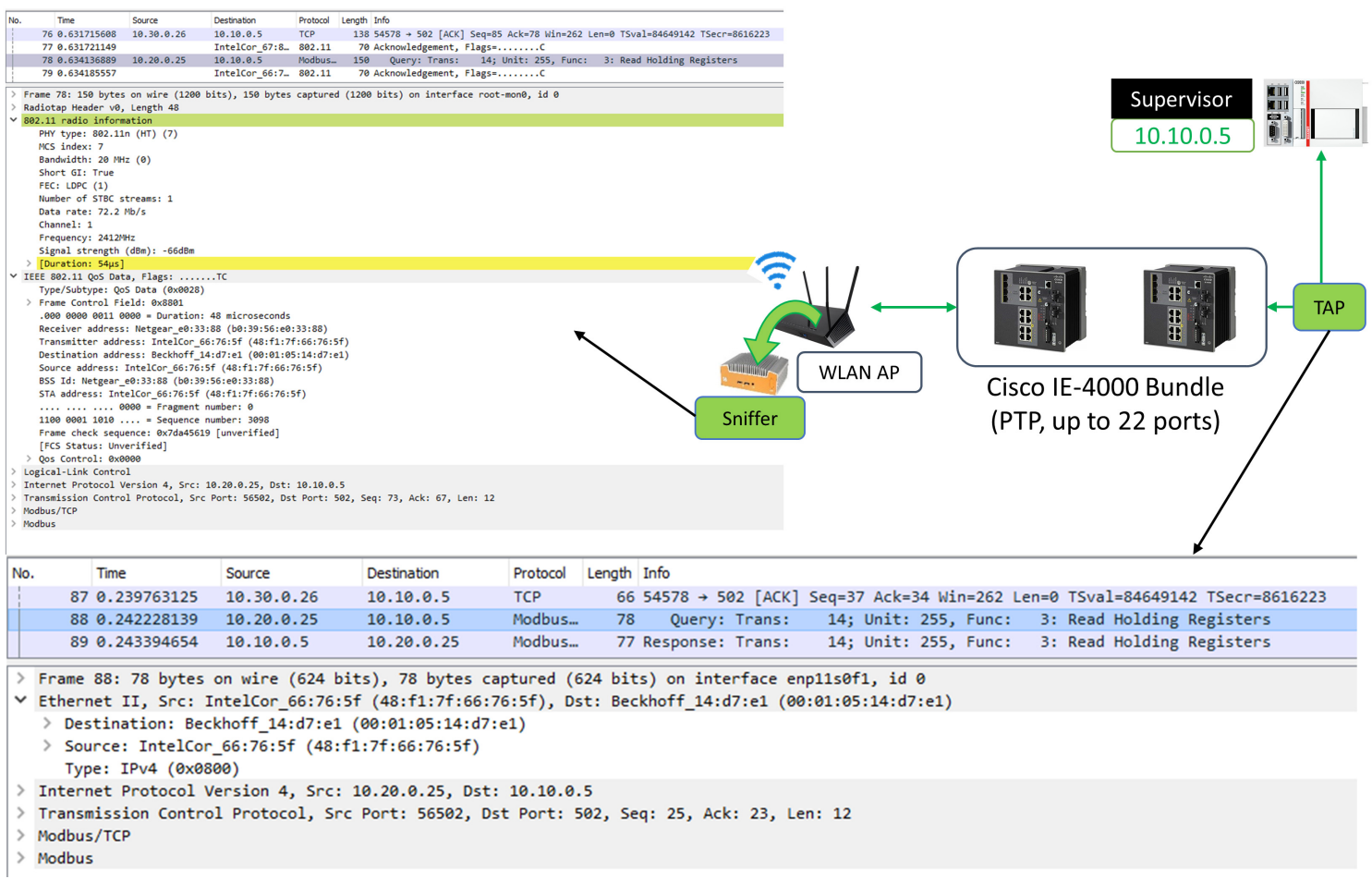

Fig. 28. Packet header samples from TAP and wireless sniffer captures

mode, the NUC's built-in wireless adapter is tuned to the AP's working channel to passively record any wireless packets sent to it. Only packet captures addressed to the co-located AP are saved in the PCAP file. The sniffer shares a common antenna with the AP so that the captured WLAN header information can indicate the channel quality experienced at the AP's receiver in UR3-AP links. Fig. 28 illustrates the captured copies of a Modbus request message recorded at the wireless sniffer (the AP is co-located with) and the TAP next to the supervisor. The wireless packet capture provides detailed information regarding transmission settings and channel quality.

\subsection{Time Synchronization}

Data timestamps are rendered at individual machines, which are synchronized with a central time server. Their formats along with the enabling tools are summarized in Table 3.

Since measurements are performed at distributed nodes and saved in multiple formats, timing information becomes a critical reference, in some cases being the sole one, to rebuild the process timeline in post data analysis. Therefore, the accuracy of distributed clocks at measurement devices determines the data quality because they are in charge of rendering record timestamps. We have introduced time synchronization approaches to guarantee the trustworthy timing information contained in measurement data. 
Table 3. Measurement data timestamp settings via PTP

\begin{tabular}{|l|l|l|l|l|l|l|l|}
\hline Data & Recorder & $\begin{array}{l}\text { Time } \\
\text { Writer }\end{array}$ & $\begin{array}{l}\text { Reference } \\
\text { Time }\end{array}$ & Format & $\begin{array}{l}\text { Time Zone, } \\
\text { Daylight Sav. }\end{array}$ & $\begin{array}{l}\text { Ordinary } \\
\text { clock (OC) }\end{array}$ & PTPv2 \\
\hline $\begin{array}{l}\text { PLC } \\
(\text { CSV) }\end{array}$ & CX2020 & $\begin{array}{l}\text { Meas. } \\
\text { POU }\end{array}$ & $\begin{array}{l}\text { PLC Task } \\
\text { Clock }\end{array}$ & $\begin{array}{l}\text { YYYY-mm-dd } \\
\text {-HH:MM:SS } \\
\text {.xxxxxx }(\mu \mathrm{s})\end{array}$ & Yes, Yes & EL6688 & L3 E2E \\
\hline $\begin{array}{l}\text { RTDE } \\
\text { (CSV) }\end{array}$ & $\begin{array}{l}\text { Central } \\
\text { Collector }\end{array}$ & Python & $\begin{array}{l}\text { Linux } \\
\text { Sys. Time }\end{array}$ & $\begin{array}{l}\text { mm/dd/YYYY, } \\
\text { HH:MM:SS, } \\
\text { xxxxxx }(\mu \mathrm{s})\end{array}$ & Yes, Yes & $\begin{array}{l}\text { NIC } \\
\text { onboard }\end{array}$ & LinuxPTP \\
\hline $\begin{array}{l}\text { TAP } \\
\text { (PCAP) }\end{array}$ & $\begin{array}{l}\text { Central } \\
\text { Collector }\end{array}$ & TShark & $\begin{array}{l}\text { Linux } \\
\text { Sys. Time }\end{array}$ & $\begin{array}{l}\text { Epoch time } \\
\text { (sec, ns-resol.) }\end{array}$ & N/A, N/A & $\begin{array}{l}\text { NIC } \\
\text { onboard }\end{array}$ & LinuxPTP \\
\hline $\begin{array}{l}\text { Wireless } \\
\text { Sniffer } \\
\text { (PCAP) }\end{array}$ & NUC & TShark & $\begin{array}{l}\text { Linux } \\
\text { Sys. Time }\end{array}$ & $\begin{array}{l}\text { Epoch time } \\
(\text { sec, ns-resol. })\end{array}$ & N/A, N/A & $\begin{array}{l}\text { NIC } \\
\text { onboard }\end{array}$ & LinuxPTP \\
\hline
\end{tabular}

\subsubsection{Precision Time Protocol}

As discussed earlier in Section 6.1, physical events are captured periodically with recording steps of milliseconds or longer. For those data, sub-millisecond level clock synchronization mechanisms, e.g., using the NTP, would be adequate to capture state transitions in system behavioral studies. However, since our study in the testbed is anticipated to investigate network events which may last only a few microseconds, it is necessary to have a more accurate time resolution in data to correctly identify various measured events in time with clear boundaries of their start and stop moments. Such a precise time measurement is essential to unveil the correlation between different data sets, especially the ones rendered from heterogeneous observers. The PTP provides the sub-microsecond level accuracy for the networked distributed clocks and widely used in timing sensitive networks. In the testbed, we have introduced PTP to synchronize measurement devices' clocks.

The testbed deploys the IEEE 1588-2008 (PTPv2) standard which defines PTP, a method to precisely synchronize computers and devices over a local area network (LAN). A Meinberg M900 PTP time server plays as the grand master clock. Individual data collectors, as introduced in Section 6.1, work as ordinary clocks (OC) in PTP which routinely update their local time based on synchronization signaling sent by the grand master. OC and the grand master are connected through the Cisco IE-4000 switch bundle which also supports PTP. These switches serve as boundary clocks (BC) which relay synchronization signaling messages as well as playing the role of intermediate time reference in the end-to-end path. These PTP appliances are connected in a tree-like topology with the root at the grand master.

Different PTP-capable hardware/software tools are deployed at OC for rendering timestamps of measurement data. For collectors running Linux, e.g., the central collector and wireless sniffer, the PTP-capable Ethernet network interface cards (NIC) serve as OC at local machines. A software tool, LinuxPTP, is used to perform the process of tuning the local system time to the reference time of the grand master [25, Chapter 23 Configuring 


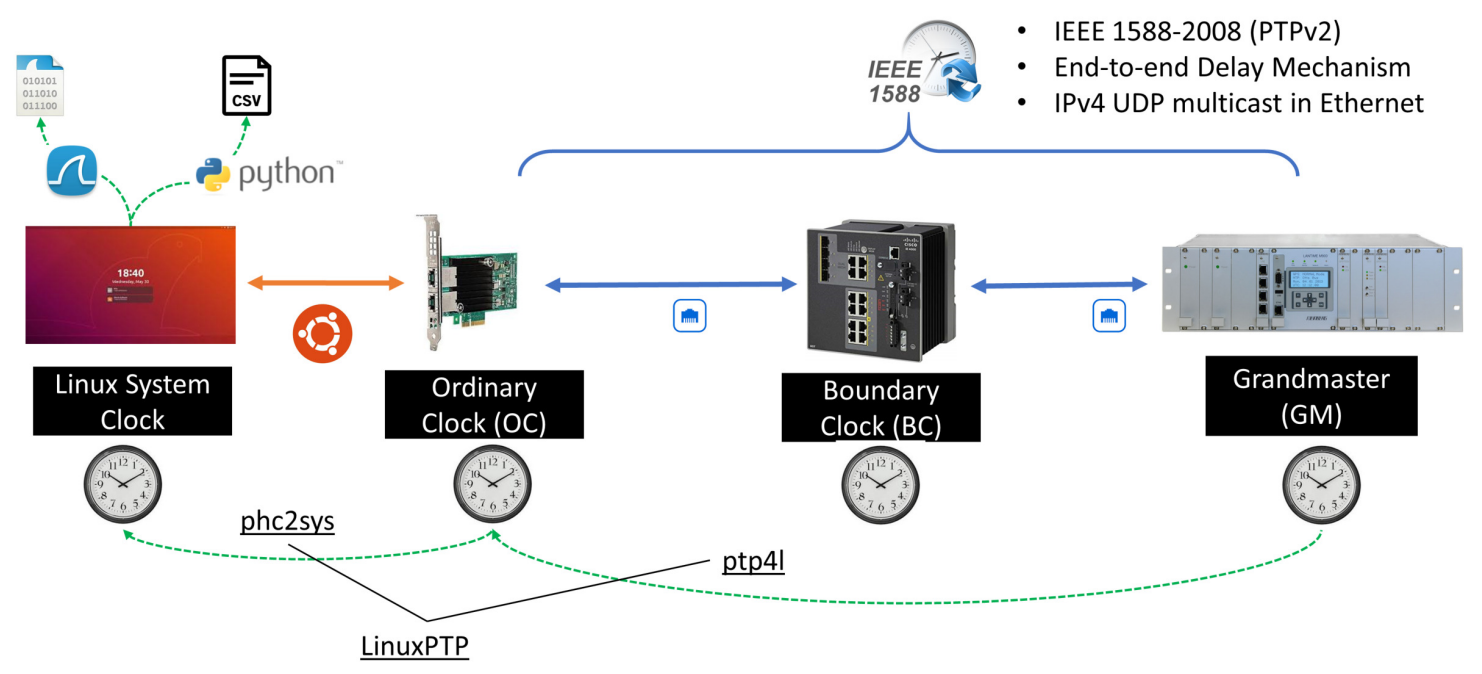

Fig. 29. PTP time synchronization using LinuxPTP

PTP using PTP4L]. Fig. 29 illustrates the synchronization process at the central controller, which runs a Ubuntu 18.04 OS. LinuxPTP contains two programs: $p t p 4 l$ and $p h c 2 s y s$. The ptp $4 l$ program is in charge of synchronizing the PTP hardware clock (PHC) on the NIC, i.e., the $\mathrm{OC}$, to the grand master using an end-to-end delay mechanism to measure the clock offset. Once OC is synchronized, the phc2sys program will then adjust the Linux system clock according to the PHC. The synchronization is performed in a repeated manner to ensure the clock offset is always managed. At the central collector, Python scripts use the datetime library to obtain the system time at the moment of writing RTDE data. For network captures from TAP devices, the recording time is stamped given the system clock reading when TShark detects the received packet in the sniffing port.

For PLC, an add-on hardware module, Beckhoff's EL6688 IEEE 1588 module, is used to enable the PTP synchronization in a similar way. The EL6688 module serves as the $\mathrm{OC}$ in the synchronization path to the grand master while providing the reference time to the connected TwinCAT devices, such as the PLC, through internal EtherCAT connections. The OC routinely measures the time offset between its clock and the PLC's system clock, which is also called the task clock (TC), and outputs the offset reading to the PLC program. At the moment when a PLC record is cached in the program, its timestamp is calculated by adjusting the current TC time based on the latest offset to the OC. A detailed explanation of PLC's PTP synchronization is presented in Appendix B.2.

\subsubsection{Timestamp Formats}

Besides implementing PTP to ensure the clock accuracy, we have further surveyed various collection tools in the testbed to verify specific timestamp formats of individual data so that the timing information can be correctly used in the analysis. The testbed is located in NIST's Gaithersburg campus where Eastern Time is used. Given specific dates in a year, 
Eastern Standard Time (EST) and Eastern Daylight Time (EDT) are used appropriately. EST(UTC-5:00) is 5 hours behind the Coordinated Universal Time (UTC); EDT (UTC4:00) has a four hour delay from UTC. The Meinberg M900 time server utilizes the UTC time which is neutral to time zones.

Table 3 illustrates timestamp formats used in individual data files. Linux machines, i.e., the central collector and wireless sniffer NUC, maintain their system clocks that are synchronized with the global time server through PTP. For network traffic data, TShark records in the epoch time which indicates the number of seconds that have elapsed since January 1 , 1970 of the UTC time. Therefore, timing information in network traffic data is immune to the setting of time zone and daylight saving time. However, the local time settings should be verified before treating production measurement data. RTDE data contain timestamps made by Python scripts at the central collector. Using the datetime library, the code outputs the local time in Eastern Time with awareness of daylight saving time. The Linux kernel takes care of the time zone setting, e.g., enabling/disabling daylight saving time, once it is configured in the system. On the contrary, for PLC records, such settings need to be manually verified and transferred to the PLC program as configuration variables, including leap seconds, time zone, and daylight saving time. In the Meas_Main module, the PLC program calls the time function to obtain the timestamp shown in local time. Appendix B.2 provides further information about rendering PTP synced timestamps in PLC data.

\section{Data Management}

A GDB is used to manage data collected from testbed measurements of both network traffic and physical operations. GDBs, as NoSQL databases, don't specify any predefined data structure or rules to enforce a fixed structure. Instead, GDBs treat individual data records as distributed node entities in a random graph and identify relationships as database components that link different nodes together. This feature allows GDBs to catch varying states and dynamics in a complex system and gradually improve data management along with better understanding of the system. In this section, we introduce graph components developed for the testbed and the data processing flow that transforms measurement results to graph entities.

\subsection{Graph Database}

The testbed GDB is built in the Neo4j desktop application. Neo4j is a native graph database which manages data and their relationships in an index-free environment. Its official query language, Cypher, is declarative, i.e., focused on what data to retrieve from the database instead of how to form queries to obtain the needed results.

\subsubsection{Graph Data Model}

In a GDB, the data model, which can be roughly analog to the "schema" of relational databases, illustrates how data records are organized and stored in a graph. However, un- 


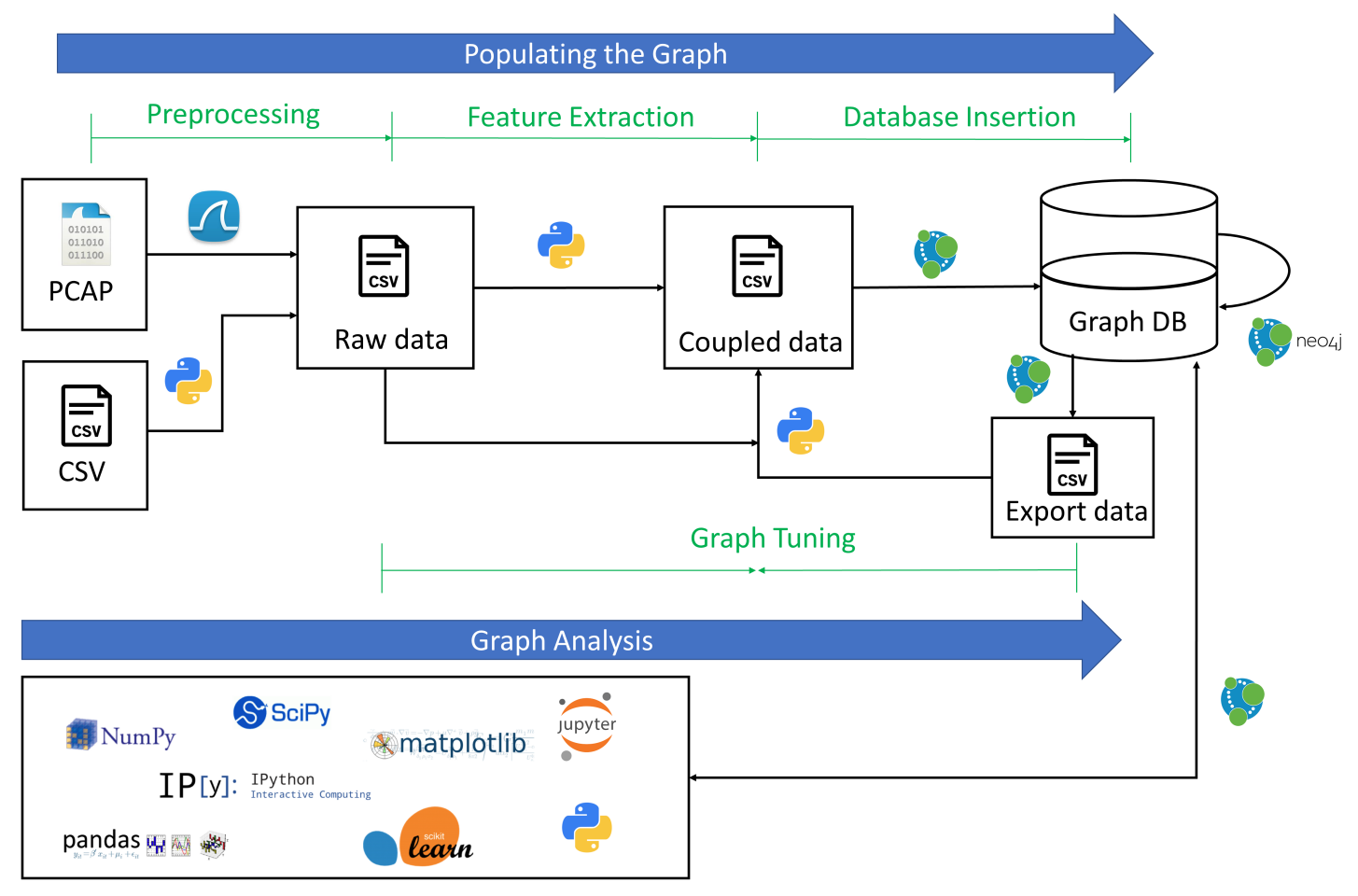

Fig. 30. Data processing flow from factory work-cell to database

like a fixed schema, the data model of GDBs has more flexibility of depicting diverse data types, content, and connections between different entities whose structure and property profile can update and evolve with more data and/or better observation. A data model contains different node types with specific properties in the graph and various relationships between them. We first identify requirements of such a data model and build a graph containing nodes and relationships that mainly exhibit information regarding networked industrial devices in a factory work-cell [6]. We further populate the previous work-cell data graph by introducing additional node types characterizing physical actions that are newly captured. Accordingly, we update the relationships, such as associating individual quality-of-service (QoS) report data made by the wireless sniffer, with the packets captured at the collocated receiver. The updated data model provides a comprehensive view of production operations, information flows, and wireless channel variations in the testbed, which facilitates further analysis work.

As shown in Fig. 31, an example is illustrated here that summarizes nodes, relationships, and their key properties used in the GDB.

\subsubsection{Building Blocks}

The graph is comprised of two classes of building blocks: nodes and relationships. The former represents testbed objects, e.g., work-cell modules and network components, and 


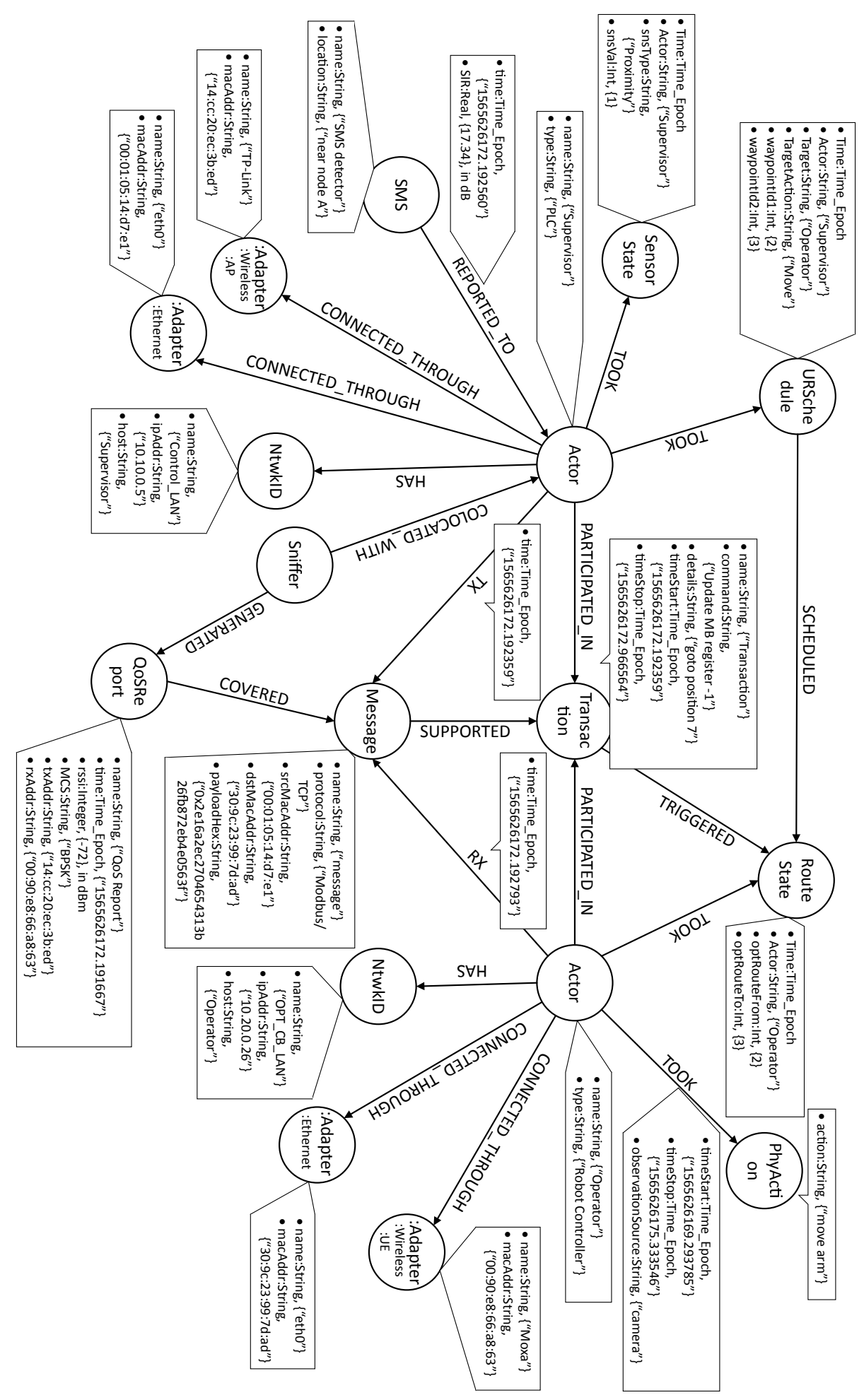

Fig. 31. The data model of the graph database used for each operational run of the testbed. 
events that are captured in the production process and network operations, e.g., machine states and communication messages; the latter is denoted as edges that link two node instances and record their relationships in terms of actions or properties. We will enumerate nodes and relationships defined for the GDB in the following parts. A thorough review of their properties can be found in Appendix C.1.

\section{Node Design}

To effectively depict testbed operations in the measurement, we define a series of node types in the graph. For different purposes, nodes can be used to identify testbed components, their states, and messages that create varying snapshots of the testbed for further analysis. They can be found in two main classes depending on what type of objects the node represents.

The class of static nodes covers testbed setup profiles, which contain testbed components, network interfaces, and their settings. These entities are normally predetermined or collected in the initialization of each measurement. They usually remain constant in each round of measurements.

Actor A physical component within the factory work-cell such as a robot, PLC, or other networked item.

NtwkID A network address item for an actor such as an Internet Protocol (IP) address.

SMS A spectrum monitoring service (SMS) observes and records significant spectral events within the work-cell and may report those events to actors within the work-cell.

Sniffer Measurement device that records all transmissions conducted over the wireless medium and includes the wireless header information for each wireless transmission detected.

Adapter Device that serves to connect an actor to a network (adapters are divided into sub-categories depending on the type of interface to a network).

Adapter:Ethernet A subcategory of adapter representing an Ethernet interface.

Adapter:Wireless A subcategory of adapter representing a wireless interface.

Adapter:Wireless:AP A subcategory of adapter representing a wireless access point interface.

Adapter:Wireless:UE A subcategory of adapter representing a wireless user equipment interface.

The class of dynamic nodes in the graph captures various system events such as machine status reports, network traffic, and information flows in the testbed. These nodes are dynamically added into the graph whose quantities and properties are determined by the real-time data in the measurement. 
Transaction A complete information exchange between two or more actors (multiple actors may participate in a transaction).

Message A network transmission event that occurs between two actors (messages are essentially packet transmissions captured at the transport layer; multiple messages support a transaction).

QoSReport Quality of service report of a message (not all messages have a QoS report).

Physical Action (PhyAction) A physical occurrence within the factory work-cell associated with Actors through multiple time-based relationships.

PhyAction:URSchedule A subcategory of PhyAction representing a schedule decision made by the supervisor PLC for a robot

PhyAction:SensorState A subcategory of PhyAction representing a real-time reading of the proximity sensor state in a CNC machine

PhyAction:RouteState A subcategory of PhyAction representing a real-time reading of the action route in a robot

\section{Graph Relationships}

A relationship in the graph denotes an action taken to associate two nodes, either homogeneous or heterogeneous ones, which shows their connections in the topology, timeline, or affiliation. We identify the following relationships in the testbed.

PARTICIPATED IN Actors will participate in transactions. A transaction exists for each logical set of messages between actors, such as the setting of a Modbus register or the sending of a command to a robot. Therefore, actors will participate in many transactions, and multiple actors may participate in a single transaction.

SUPPORTED Messages (i.e., packets between actors) are associated with transactions through the SUPPORTED relationship. Depending on the protocol and the quality of the channel, a single transaction could have one or many messages connected through this relationship.

TX/RX An actor may either transmit (TX) or receive (RX) a message. Both the TX and RX relationships contain a timestamp in the format of an epoch time which is a floating point number in seconds since January 1, 1970, with a resolution of microseconds.

TOOK When an actor performs a physical action, a TOOK relationship is created between the actor and the physical action node. This relationship contains start and stop time properties as well as the source of the observation such as a networked camera. 
REPORTED_TO An SMS may be a passive or active listener within a work-cell. When an SMS operates as an active listener, spectral reports from the SMS may be sent to an actor such that the actor can respond intelligently to the spectral event. Reports from an SMS to an actor are captured within this relationship.

COVERED A wireless sniffer keeps monitoring the working wireless channel(s) and extracts the real-time link QoS information from the sniffed wireless packets, such as the received signal strength indicator (RSSI). A COVERED relationship links the QoSReport node with the concurrent Message node received at the same spot. Not all Message nodes have such a relationship with QoSReport which depends on the availability of the sniffer collocated with the receiver and any wireless sniffer data reported during the transmission.

Other relationships shown in Fig. 31 but not explained above are considered self-explanatory.

\section{Closer Examination}

The graph data model is designed in a way where nodes and relationships are centered around Actors. Actors have dual roles in the work-cell operations. In the factory system, Actors participate in the production operations. In the example of Fig. 31, two Actor nodes are presented. In this case, Actor "Supervisor" is the supervisory controller, and Actor "Operator" is a robot arm. The Supervisor schedules the production, collects the other Actors' states, and hosts supportive services, such as SMS. The Operator follows the instructions of the Supervisor and moves parts between work stations. Meanwhile, Actors also act as communication nodes which exchange messages between each other through various network interfaces. In Fig. 31, Actors participate in a transaction, which, in this example, is a Modbus/TCP exchange. The transaction itself is associated with one or more messages (i.e., packets). Each message associated with a transaction manifests itself as a node in the graph. Multiple message nodes will exist for each transaction. Additionally, QoS reports may be associated with each actor node through a collocated sniffer node.

Dynamic event nodes in the measurement, i.e., physical actions, network messages, information transactions, and QoSReport records, have timestamps representing "measurement time" of the recorded events. Once a new event occurs, a proper relationship would be added between the actor and the physical/network event node. All timestamps are accurately synchronized to the grand-master clock.

\subsection{Data Importing Pipeline}

A multi-stage workflow is deployed to feed the graph with instances of nodes, relationships, and their properties that are extracted from measurement data, as shown in Fig. 30. In the data set, network data are captured from distributed probes in the selected links and stored in PCAP files, while operational data that come from different PLC and robot controllers are stored in comma separated value (CSV) files. The whole data process contains 
four steps including preprocessing, feature extraction, database insertion, and graph tuning. Such conversion from raw measurement data to a ready-to-go graph has been automated by scripts deployed in the central collector, which maintains data repositories and deploys the Neo4j desktop application. Functions and operation features in individual steps are discussed next.

\subsubsection{Data Preprocessing}

Data preprocessing is the beginning of the whole process. In this stage, measurement data from various sources are verified, cleaned, and formatted in a way to facilitate the following processing and interpretation. As fore-mentioned, measurement data contains records collected from heterogeneous modules/devices in the testbed, which may utilize different data types, sampling rates, time and metric resolution, as well as file formats. For example, different machines may store records' timing information in various timestamp formats depending on local clock preference. In the process, we have unified the data set's time format, i.e., using the time epoch with the microsecond resolution. Besides, we have also deployed packet filters to reduce the size of output data for the following steps, e.g., removing uncorrelated packet captures. For example, when treating wireless sniffer data in experiments with interference links, we have managed to reduce the size of sniffer data, which was originally of gigabytes, to only a few megabytes with only signaling handshakes in the studied links.

\subsubsection{Feature Extraction}

Feature extraction refers to the process of extracting relevant information from measurement data to prepare the data for insertion into the database. Nodes and relationships are defined by a set of features that share common views. We have developed Bash and Python scripts that pick the desired features to produce CSV files that are ready for insertion into the Neo4j database. In this step, a Bash script runs TShark to extract fields of protocol headers in packet captures and save the field information into CSV files. Each row in these CSV files will lead to one Message node instance being created which is one packet copy at the sender or receiver. A Python script was also used to detect physical action changes and label those state switching moments, which were associated with communication messages.

\subsubsection{Graph Insertion}

We load the prepared data into the Neo4j GDB using bulk importing, which can create multiple new nodes and/or relationships by reading a CSV file once. Neo4j uses Cypher to construct GDB queries that import data. As the output of feature extraction, each CSV line contains the information for creating one new node entity and/or pairing two nodes in a new relationship. Properties of new entities can be assigned explicitly by the column values of records or inferred from predetermined rules such as some fixed combination of 
nodes and edges in the graph. Multiple types of nodes can be created from the same data file using one common node template in which each node type has its own subgroup of properties. For example, Modbus and ADS packets use the same Message node structure in our graph to manage the common transmission information such as IP addresses and TCP session identification. Meanwhile, each of these Messages maintains its own application layer header information in the node properties, e.g., Modbus register addresses and ADS function codes.

\subsubsection{Graph Tuning}

Graph tuning refers to any additional modification in the graph after CSV data has been imported. This step treats a number of cases where the unexplored raw data is coupled with the imported one to extend/improve the graph. First, in the additive insertion cases, i.e., when new data is to be added, this step links the newly added nodes to the existing ones and creates necessary relationships between them. For example, time series data often uses this method to link consecutive event nodes in the recorded process. Second, there are cases when further insights of system properties can be obtained by querying existing database records which lead to new nodes and relationships. For example, Transaction nodes are built upon Message nodes who participate in the same application transactions; Message nodes themselves are also the summary of packet data, i.e., packet copies at link transceivers. Third, it can serve as one input to the feature extraction step by providing existing graph information for purposes such as coupling data records. For example, coupling QoS reports and Messages in their observation windows used to be an extremely time-consuming process. On one hand, each Message raw data, i.e., the transmitter or receiver copy, contains only half of the transmission time window information. On the other hand, the Cypher query takes a long time to find all eligible relationships as Neo4j would generate a huge Cartesian product when treating the large sample set. We solved this issue by obtaining qualified Message nodes and feeding them into feature extraction where a more efficient Python script finds all Message-QoS Report pairs, and later, presents them in the graph as new COVERED relationships.

The above four steps can perform multiple iterations to treat data and refine the graph according to the data complexity and requirements. To further demonstrate such a process in treating experiment data, we enumerate various techniques used in the testbed and show them in Appendix C.

\section{Data Analysis \& Graph Exploration}

\subsection{Graph Database Schema}

Once the Neo4j database is populated with testbed data, we can apply Cypher queries to explore the constructed graph and extract information for assessing the work-cell performance and visualizing selected network and operational events within the work-cell. More advance data science tools, such as Python's Pandas and Scikit-learn packages, can also 


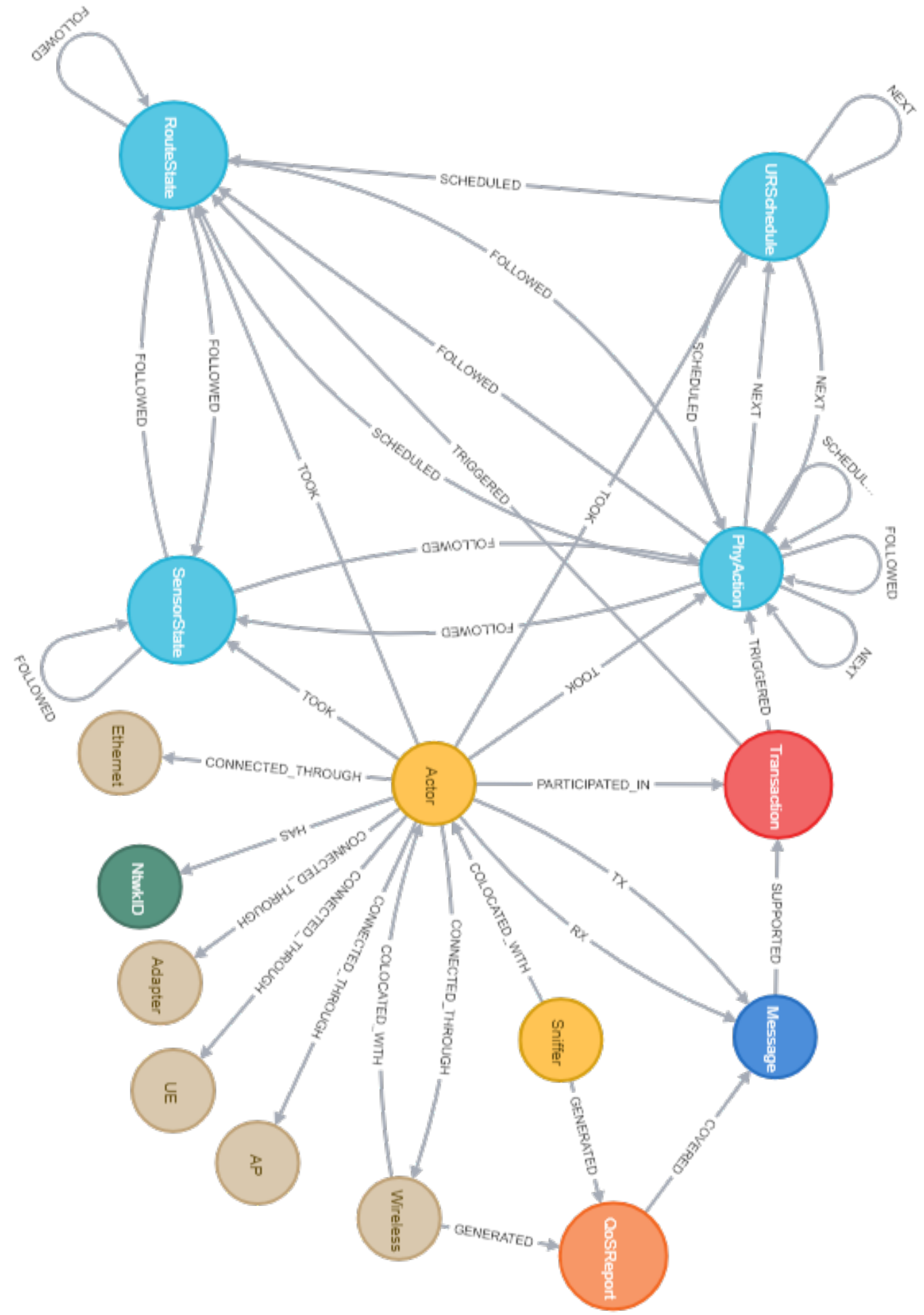

Fig. 32. Realized schema of the graph database fully populated after capturing network and operational data from the industrial wireless testbed. 
be introduced using Python-Neo4j interfaces, details of which can be referred to in Appendix C.2.4.

Fig. 32 shows the graph schema as a quick check of the Neo4j graph elements (as proposed in Fig. 31) using the query command, call db.schema.visualization().

\subsection{Experiment Configurations}

Section 4 and 5 have introduced general configurations on the production modules and network components, respectively. Additional configuration settings are provided here for testbed experiments of evaluating the work-cell performance under different network conditions.

During each run of an experimental scenario, the production of 20 parts was emulated. Each part went through a directed tooling path, i.e., $<$ Queue IN $>-<C N C 1>-<$ CNC2 $>-<C N C 3>-<C N C 4>-<$ Queue OUT $>$, which resulted in around 12 minutes of network activity.

We performed four different experimental cases with respect to the communications network settings, namely,

1. Wired baseline: All links are connected using Ethernet cables to act as a benchmark for performance comparison (see Fig. 22 for the wired architecture and Fig. 64 for the measurement diagram);

2. Wireless scenarios: Two wireless links are used to connect the robot controllers and the wireless AP that is connected to all the other actors in the testbed (see Fig. 23 for the wireless architecture and Fig. 65 for the measurement diagram). The wireless nodes are Intel NUC devices with IEEE $802.11 \mathrm{~b} / \mathrm{g} / \mathrm{n}$ interfaces.

(a) Wireless baseline: The robots' traffic is the only traffic transferred over the wireless network;

(b) Wireless communications with a single interference pair: One additional pair of Intel NUC devices forms an interference link in which 2500 packets per second (pps) wireless traffic is generated in the same working WLAN channel as the robot links. The interference link packets have the size of 1000 Bytes.

(c) Wireless communications with two interference pairs: Two additional pairs of Intel NUC devices form two interference links each of which generates 1250 packets per second (pps) wireless traffic in the same working WLAN channel as the robot links. The interference link packets have the size of 1000 Bytes.

As shown in the measurement diagrams of Fig. 64 and Fig. 65, we collected network capture data from TAP devices in the testbed. In wireless scenarios, we also collected wireless packet captures at the wireless sniffer to describe the WLAN channel condition. Data from the supervisor was also collected which included the system states and the supervisory commands. In addition, data from the robots was used to describe the physical actions taken. 


\subsection{Preliminary Results}

In this subsection, we use the extracted data from the GDB to study the impact of the wireless communications on the physical action performance. The results have been partially reported in our earlier publications [5], [6], and [7]. The data is being prepared for public access, e.g., the data presented in [6] is available in [26]. We focus our analysis on the URSchedule and RouteState progress over time where URSchedule is the dynamic node to represent a physical action decision at the supervisor and RouteState is the dynamic node to represent a physical action command received by one of the robots where the command parameters are stored at the robot registers. Note here that the transaction between a robot controller and the supervisor is initiated by a request message from the robot controller and terminated by correctly receiving a response message from the supervisor to the robot controller as well.

The supervisor makes decisions based on available information about the testbed. Once it makes a decision, it is reflected on the value of the URSchedule. We define the supervisor processing time as the time from the instant the decision is taken to the instant when the wireless transaction is initiated to request a new physical action and it is denoted by $T_{\text {Sup }}$. Then, the transaction latency is the total time spent by all the wireless packets corresponding to an action such that it is the time between the instant, at which the wireless transaction is initiated by the robot controller to require a new action until the data arrives from the supervisor at the intended robot controller. The wireless transaction latency is denoted by $T_{\mathrm{W}}$. The robot processing time is the time between the instant the wireless data is received by the robot controller to the instant when the required action is updated in the RouteState register indicating the physical action starts. The robot processing time is denoted by $T_{\mathrm{Rob}}$. The total physical action time, which represents the time needed for a supervisor command to be reflected at the corresponding robot, is denoted by $T_{\mathrm{Act}}$ and evaluated through

$$
T_{\mathrm{Act}}=T_{\mathrm{Sup}}+T_{\mathrm{W}}+T_{\mathrm{Rob}} \text {. }
$$

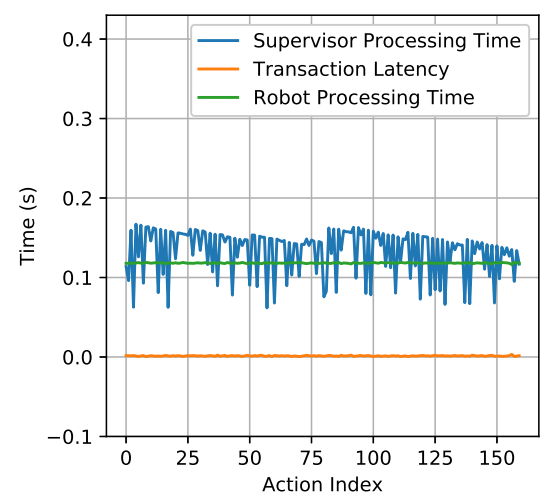

Fig. 33. Wired baseline physical action time

In Fig.33-37, we present the values of the three components of the total physical action time for each run of the testbed. The horizontal axis represents the action index for all the 


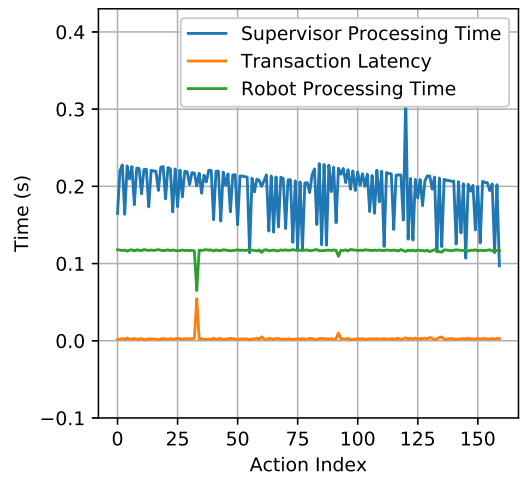

Fig. 34. Wireless baseline physical action time

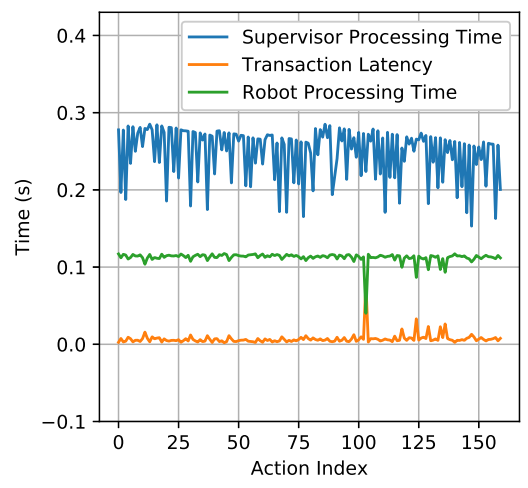

Fig. 35. Wireless with 2500 pps traffic physical action time (run 1)

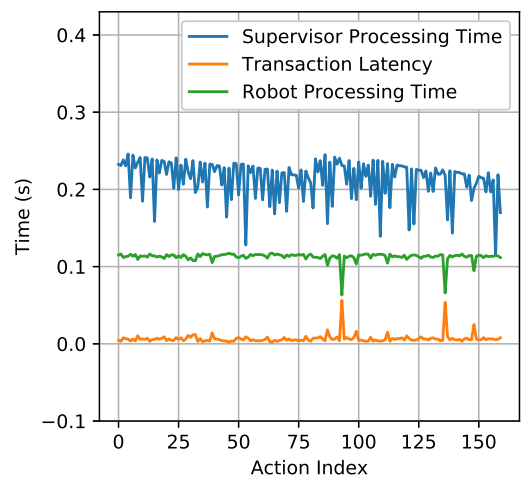

Fig. 36. Wireless with 2500 pps traffic physical action time (run 2)

operator and inspector actions, while the corresponding time components are shown in the vertical figure axis.

We present the normalized histograms of the transaction latency and the total physical action time in Figs. 38 and 39, respectively. Interested readers can refer to [7] for further discussions on the key factors to these metrics. 


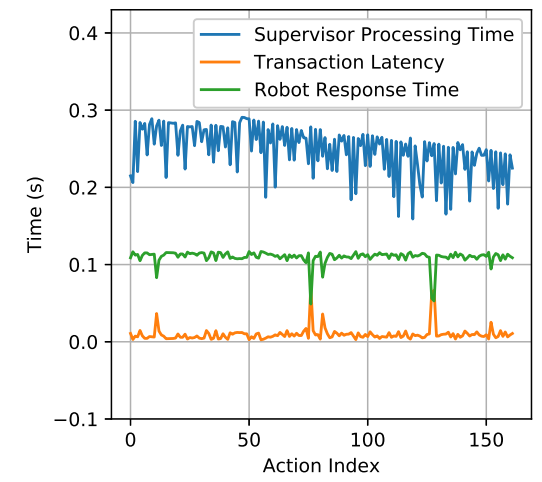

Fig. 37. Wireless with $2 \times 1250$ packets/s traffic physical action time

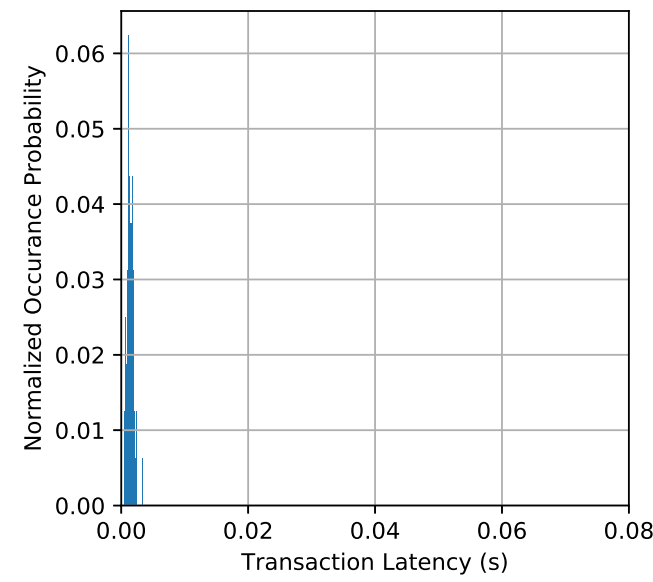

a Wired Baseline

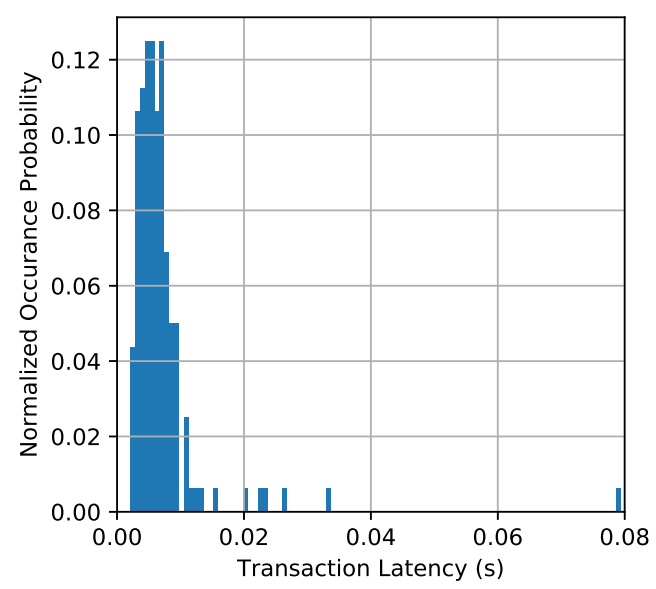

c With 2500 pps Traffic

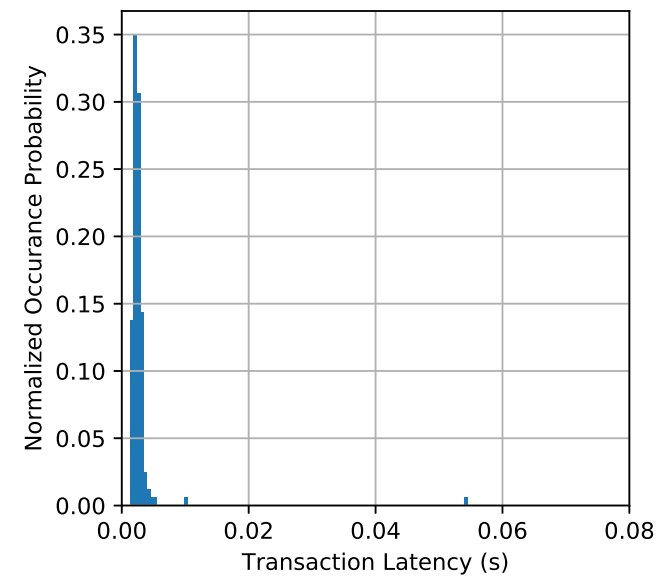

b Wireless Baseline



d With 2x1250 pps Traffic

Fig. 38. Histograms of Transaction Latency for Various Experimental Scenarios 


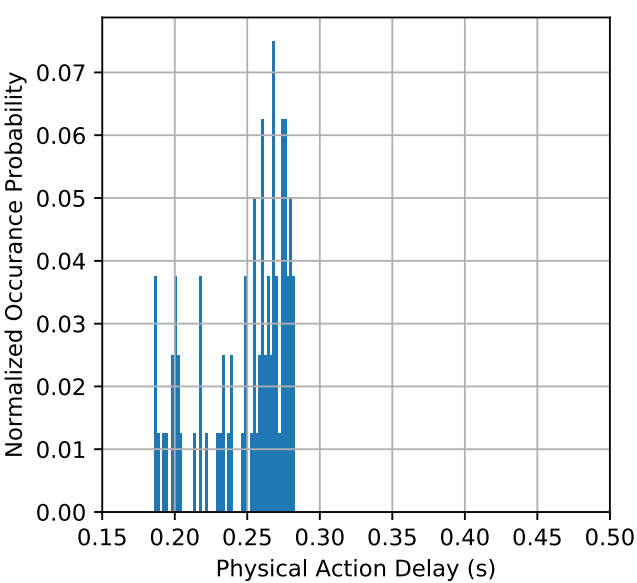

a Wired Baseline

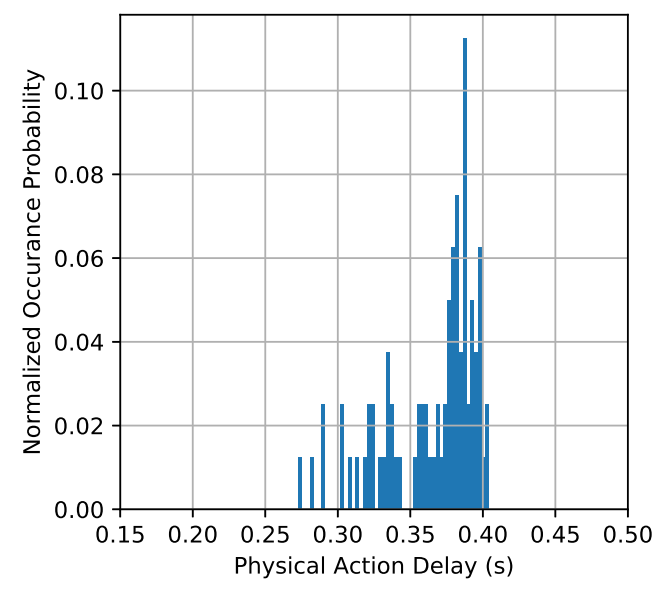

c With 2500 pps Traffic

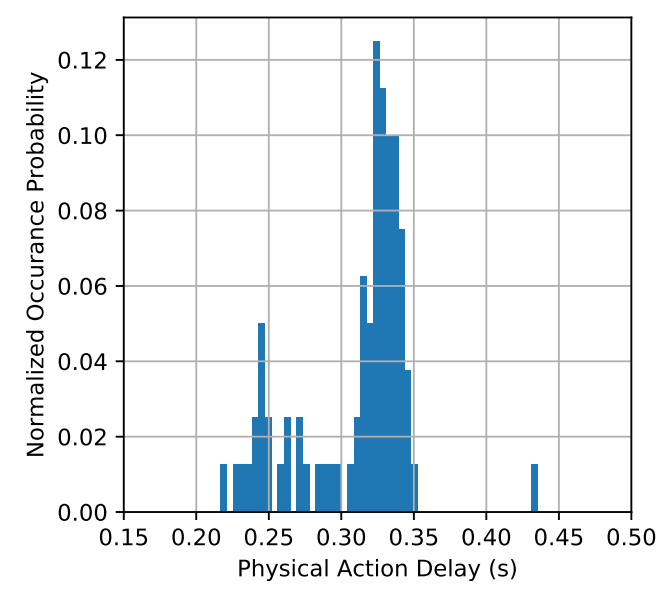

b Wireless Baseline

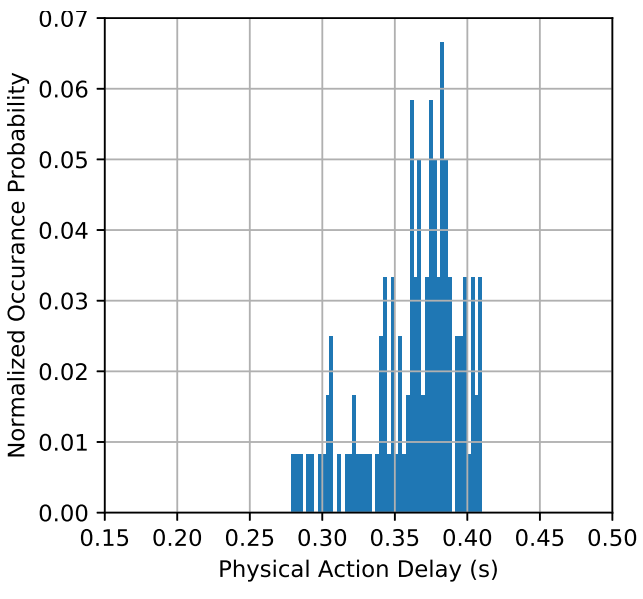

d With 2x1250 pps Traffic

Fig. 39. Histograms of Physical Action Time for Various Experimental Scenarios

\section{Conclusion}

We have presented a new testbed design idea in the study of wireless techniques in support of industrial operations. The proposed collaborative robot work-cell scenario represents a wide spectrum of manufacturing activities with data transmission needs to coordinate their work. The measurement approach and data processing pipeline based on the graph database provide flexibility in exploring data across the CPS domains. The testbed has been used in our research work in collaboration with industrial partners to validate and showcase the capability of recent WLAN products in serving industrial applications. More measurement events are planned for this testbed to verify emerging industrial wireless techniques, such as wireless TSN and the next generation WLAN solutions. Further progress will be reported in future releases. 


\section{Acknowledgments}

The authors would like to thank Jing Geng at the University of Maryland for helping us to develop Bash scripts of using TShark in dissecting packet captures. We would like to also thank our industrial partners, Dave Cavalcanti and Susruth Sudhakaran from Intel Labs, for their contributions in providing the Intel NUC devices. We would like to thank Timothy Zimmerman, Ya-Shian Li-Baboud, CheeYee Tang, Frederick M. Proctor, and Keith Stouffer at NIST, for their continuous support and valuable suggestions on the testbed development.

\section{References}

[1] Liu Y, Kashef M, Lee KB, Benmohamed L, Candell R (2019) Wireless network design for emerging IIoT applications: Reference framework and use cases. Proceedings of the IEEE 107(6):1166-1192.

[2] Montgomery K, Candell R, Liu Y, Hany M (2019) Wireless User Requirements for the Factory Work-cell. The National Institute of Standards and Technology (NIST, Technical report. https://doi.org/10.6028/NIST.AMS.300-8. URL https://www.nist. gov/publications/wireless-user-requirements-factory-workcell

[3] Candell R, et al. (2018) Guide to industrial wireless systems deployments. National Institute of Standards and Technology Gaithersburg, MD, Technical report. https://doi.org/10.6028/NIST.AMS.300-4. URL http://nvlpubs.nist.gov/nistpubs/ams/ NIST.AMS.300-4.pdf

[4] NIST (2018) Trustworthy systems, components, and data for smart manufacturing program, https://www.nist.gov/programs-projects/ trustworthy-systems-components-and-data-smart-manufacturing-program. Accessed: 2020-09-30.

[5] Liu Y, Candell R, Kashef M, Montgomery K (2019) A collaborative work cell testbed for industrial wireless communications - the baseline design. 2019 IEEE 28th International Symposium on Industrial Electronics (ISIE), pp 1315-1321. https://doi.org/ 10.1109/ISIE.2019.8781524

[6] Candell R, Kashef M, Liu Y, Montgomery K, Foufou S (2020) A Graph Database Approach to Wireless IIoT Workcell Performance Evaluation. 2020 IEEE International Conference on Industrial Technology (ICIT) (IEEE), pp 251-258. https://doi.org/10.1109/ICIT45562.2020.9067199. URL https://ieeexplore.ieee.org/ document/9067199/

[7] Kashef M, Liu Y, Montgomery K, Candell R (2020) Wireless cyber-physical systems performance evaluation through a graph database approach. Journal of Computing and Information Science in Engineering :1-19,URL https://doi.org/10.1115/1. 4048205.

[8] NIST (2018) Cybersecurity for smart manufacturing systems, https://www.nist.gov/ 
programs-projects/cybersecurity-smart-manufacturing-systems. Accessed: 2020-0930.

[9] Candell R, et al. (2017) Industrial wireless systems radio propagation measurements. the National Institute of Standards and Technology (NIST, Technical report. https://doi.org/10.6028/nist.tn.1951

[10] Li H, et al. (2019) Design space exploration for wireless-integrated factory automation systems. 2019 15th IEEE International Workshop on Factory Communication Systems (WFCS), pp 1-8.

[11] Geng J, et al. (2020) Integrating field measurements into a model-based simulator for industrial communication networks. 2020 16th IEEE International Conference on Factory Communication Systems (WFCS), pp 1-8.

[12] Aminian B, Araujo J, Johansson M, Johansson KH GISOO: A virtual testbed for wireless cyber-physical systems. IECON 2013 - 39th Annual Conference of the IEEE Industrial Electronics Society (IEEE), pp 5588-5593. https://doi.org/10.1109/IECON. 2013.6700049

[13] Jecan E, Pop C, Padrah Z, Ratiu O, Puschita E A dual-standard solution for industrial Wireless Sensor Network deployment: Experimental testbed and performance evaluation. 2018 14th IEEE International Workshop on Factory Communication Systems (WFCS) (IEEE), pp 1-9. https://doi.org/10.1109/WFCS.2018.8402360

[14] Ding Y, et al. Experimental investigation of the packet loss rate of wireless industrial networks in real industrial environments. 2015 IEEE International Conference on Information and Automation (IEEE), pp 1048-1053. https://doi.org/10.1109/ICInfA. 2015.7279441

[15] Liu Q, et al. (2018) Design and Evaluation of a Real Time Physiological Signals Acquisition System Implemented in Multi-Operating Rooms for Anesthesia. Journal of Medical Systems 42(8):148. https://doi.org/10.1007/s10916-018-0999-1

[16] Fink J, Ribeiro A, Kumar V (2013) Robust Control of Mobility and Communications in Autonomous Robot Teams. IEEE Access 1:290-309. https://doi.org/ 10.1109/ACCESS.2013.2262013

[17] Liang W, et al. (2019) WIA-FA and Its Applications to Digital Factory: A Wireless Network Solution for Factory Automation. Proceedings of the IEEE 107(6):10531073. https://doi.org/10.1109/JPROC.2019.2897627

[18] Candell R (2015) A Research Framework for Industrial Wireless Deployments. Proceedings of 2015 ISA Instrumentation Symposium URL https://www.researchgate.net/publication/285396923_A_Research_Framework for_Industrial_Wireless_Deployments.

[19] Liu Y, Candell R, Lee K, Moayeri N (2016) A simulation framework for industrial wireless networks and process control systems. 2016 IEEE World Conference on Factory Communication Systems (WFCS) (IEEE), pp 1-11. https://doi.org/ 10.1109/WFCS.2016.7496495

[20] Technical Committee (2008) IEEE Std 1588-2008, IEEE Standard for a Precision Clock Synchronization Protocol for Networked Measurement and Control Systems. 
Society https://doi.org/10.1109/IEEESTD.2008.4579760

[21] Schick IC, Gershwin SB, Kim J (2005) Quality/Quantity Modeling and Analysis of Production Lines Subject to Uncertainty, Phase I, Final Report. Massachusetts Institute of Technology USA, Technical report. Also available as http: //web.mit.edu/manuf-sys/www/oldcell1/papers/GM_PhaseI_FinalReport-2005.pdf. Accessed: 10/01/2020.

[22] Kim J, Gershwin SB (2005) Integrated quality and quantity modeling of a production line. OR Spectrum 27(2-3):287-314.

[23] iPerf (2019) iPerf2 - A tool that measures network performance of TCP/UDP, https: //sourceforge.net/projects/iperf2/. Accessed: 2020-10-14.

[24] Universal Robots (2020) Real-time data exchange (RTDE) guide, https: //www.universal-robots.com/articles/ur/real-time-data-exchange-rtde-guide/. Accessed: 2020-09-30.

[25] Red Hat (2017) Deployment, Configuration and Administration of Red Hat Enterprise Linux 6. Red Hat USA, Technical report. Also available as https://access.redhat. com/documentation/en-us/red_hat_enterprise_linux/6/html/deployment_guide/index. Accessed: 10/01/2020.

[26] Montgomery K (2020) Measurement and Processed Data From A Graph Database Approach to Wireless IIoT Work-cell Performance Evaluation, https://data.nist.gov/ od/id/mds2-2242. Accessed: 2020-09-30.

[27] Acromag (2020) Introduction to MODBUS TCP/IP. Acromag USA, Technical report. Also available as https:/www.acromag.com/wp-content/uploads/2019/08/ White-Paper-Introduction-to-ModbusTCP_765B-.pdf. Accessed: 10/01/2020.

[28] Lyon GF (2009) Nmap Network Scanning [Online Edition] (Nmap Project), . Available as https://nmap.org/book/toc.html. Accessed: 10/01/2020.

[29] Beckhoff (2020) TwinCAT ADS/AMS - Specification. Beckhoff Information System USA, Technical report. Also available as https://infosys.beckhoff.com/english. php?content $=. . /$ content $/ 1033 /$ tcadsamsspec $/ \mathrm{html} /$ tcadsamsspec_adscmds.htm\&id= 1605425048011779071. Accessed: 10/01/2020.

[30] Beckhoff (2018) IEEE 1588 external synchronization interface (EL6688). Beckhoff Information System USA, Technical report. Also available as https://www.beckhoff. com/english.asp?ethercat/el6688.htm. Accessed: 10/01/2020.

[31] WireShark (2020) tshark - Dump and analyze network traffic. wireshark.org USA, Technical report. Also available as https:/www.wireshark.org/docs/man-pages/ tshark.html. Accessed: 10/01/2020.

[32] Universal Robots (2020) UR CB3, https://www.universal-robots.com/cb3/. Accessed: 2020-09-30.

[33] Robotiq (2020) 2F-85 and 2F-140 Grippers, https://robotiq.com/products/ 2f85-140-adaptive-robot-gripper. Accessed: 2020-09-30.

[34] OnRobot (2020) 6 axis Force Torque Sensor, https://onrobot.com/en/products/ hex-6-axis-force-torque-sensor. Accessed: 2020-09-30.

[35] Beckhoff (2020) CX2020 Basic CPU Module, https://www.beckhoff.com/english. 
asp?embedded_pc/cx2020.htm. Accessed: 2020-09-30.

[36] Beckhoff (2020) CX9020 Basic CPU Module, https://www.beckhoff.com/english. asp?embedded_pc/cx9020.htm. Accessed: 2020-09-30.

[37] Shuttle (2020) Entry-level Redefined: Fanless 1 liter PC with Intel Gemini Lake (DL10J), http://global.shuttle.com/products/productsSpec?productId=2281. Accessed: 2020-09-30.

[38] Meinberg (2020) LANTIME M900/PTP, https://www.meinbergglobal.com/english/ products/modular-3u-ieee-1588-grandmaster-clock.htm. Accessed: 2020-09-30. 


\section{Appendices}

\section{A. Communication Messages in the Testbed}

In this appendix, we provide the basic knowledge for understanding various network packet formats that have been used in the testbed measurements and analysis. Protocol header information and field values are verified using WireShark packet captures from the testbed network links.

\section{A.1 Basic Packet Format}

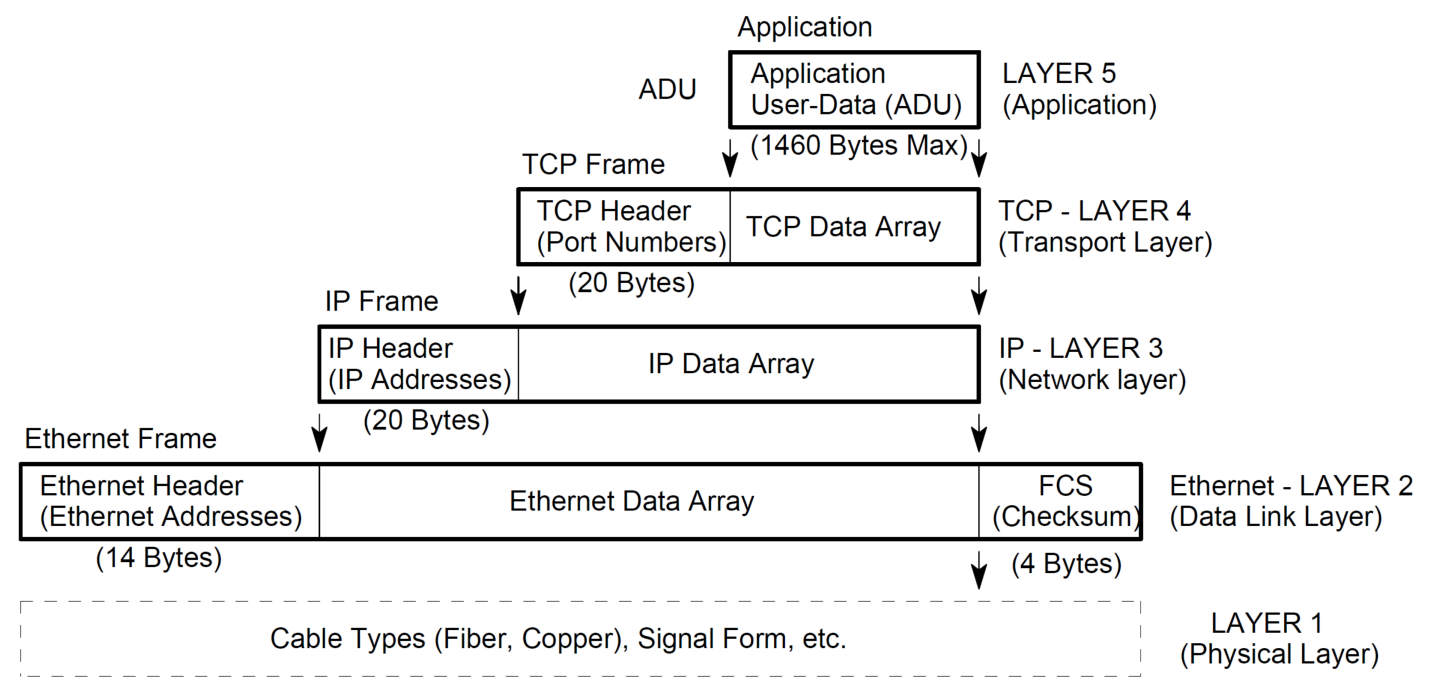

Fig. 40. Construction of a TCP/IP-Ethernet data packet (reprinted from [27])

Application messages refer to data exchanged between networked testbed components for work-cell coordination and other testbed functions. The control information regarding individual protocol layers is usually concatenated into the packet's headers and sent along with data in the medium, such as Ethernet links or wireless channels. Following the OSI's 7-layer model, the analysis on testbed messages uses the information of following layers including application, transport, network, MAC, and PHY.

Communication protocols such as ADS, Modbus, RDP, and intra-robot messages use TCP in the transport layer and IP in the network layer. These TCP/IP packets are originally designed to carry application data in Ethernet links. Most of network components in the testbed are equipped with one or more Ethernet ports as the main network interface to the wired network. Fig. 40 illustrates the layered packet format and header fields of individual 


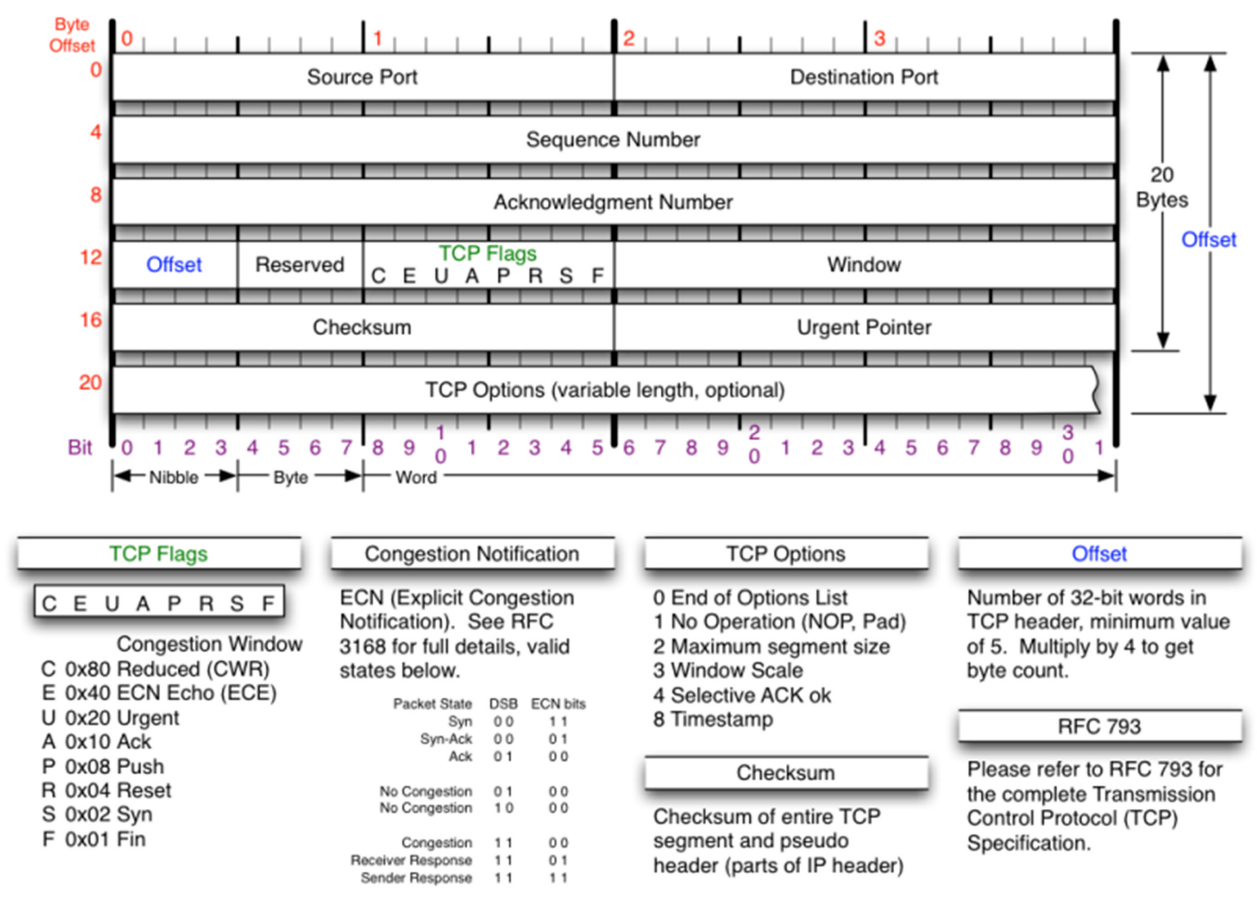

Fig. 41. TCP header (reprinted from [28, TCP/IP Reference])

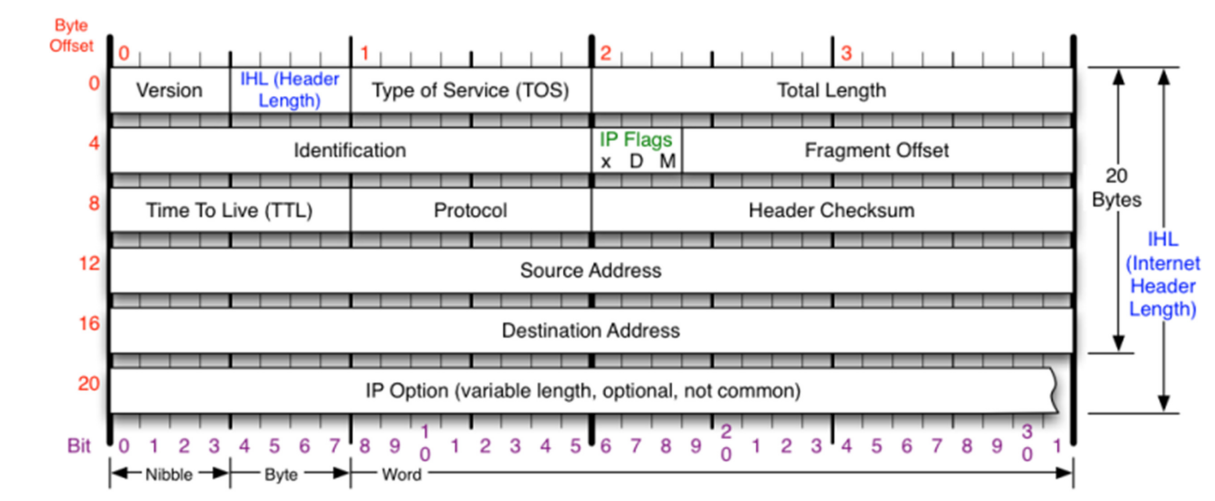

\begin{tabular}{l}
\hline \multicolumn{1}{c}{ Version } \\
Version of IP Protocol. 4 and \\
6 are valid. This diagram \\
represents version 4 \\
structure only. \\
\hline \multicolumn{1}{c}{ Header Length } \\
Number of 32-bit words in \\
TCP header, minimum value \\
of 5 . Multiply by 4 to get byte \\
count.
\end{tabular}
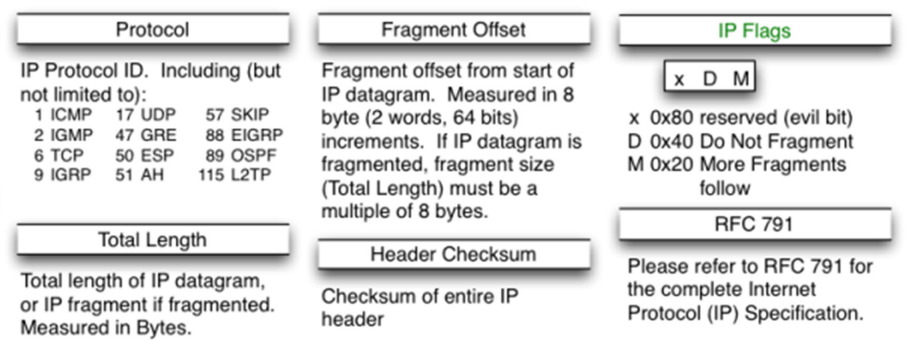

Fig. 42. IP header (reprinted from [28, TCP/IP Reference]) 
layers in a typical TCP/IP data packet through Ethernet. If such packets transmit wirelessly, the wireless protocol header will replace the Ethernet header. The upper layer meta information and message data remain intact. Fig. 41 and Fig. 42 illustrate details of TCP and IP header information, respectively.

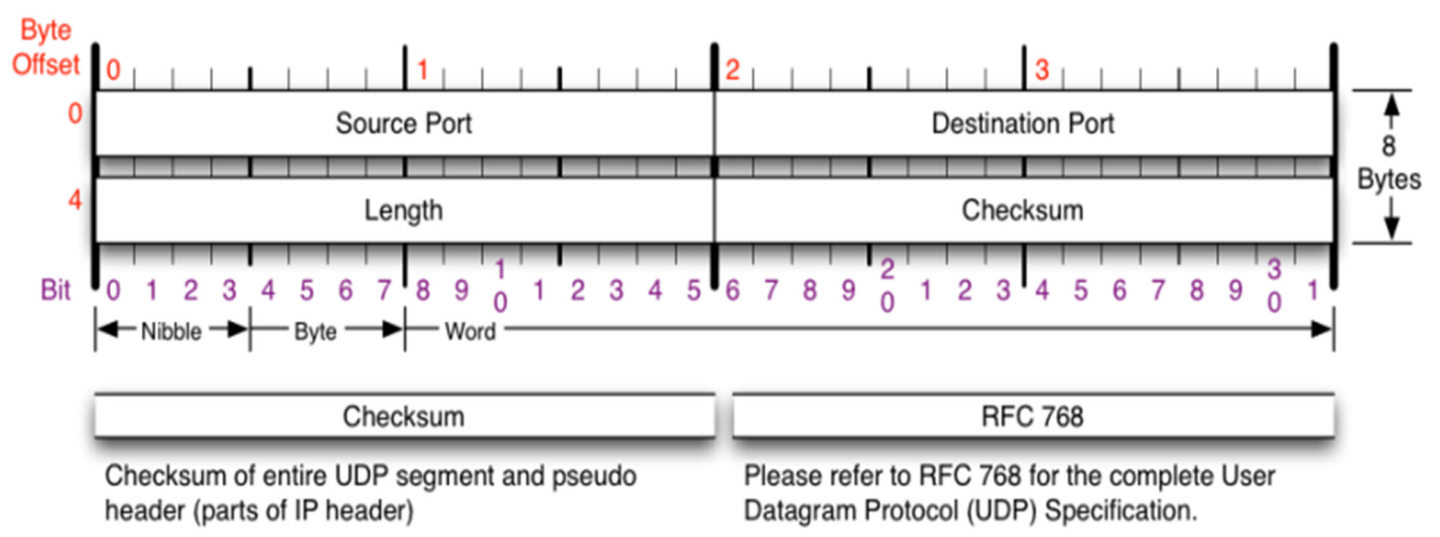

Fig. 43. UDP header (reprinted from $[28, T C P / I P$ Reference $]$ )

UDP/IP packets also carry a part of the testbed traffic such as time synchronization messages in both NTP and PTPv2 protocols. Similar to the TCP-based packet format, UDP-based packets contain the UDP header information, as shown in Fig. 43, in the transport layer.

\section{A.2 ADS/AMS}

Beckhoff's TwinCAT devices, such as PLCs including both CX2020 and CX9020 and their peripheral terminal modules (if available), use a proprietary communication protocol to exchange data and control messages with each other. Specifically, their messages are formatted as predetermined industrial functions and command codes, named ADS commands. A unique ID called AMS Net ID is used to identify each ADS node in the TwinCAT network. By default, the AMS Net ID is defined as "a.b.c.d.e.f" where "a.b.c.d" is the device's IPv4 address and the last two segments "e.f" are usually set as "1.1". If the ADS node has multiple AMS interfaces or multiple ADS nodes belong to a subnet, the extra segments can help specify the hierarchy of TwinCAT network related with the industrial system architecture. In Beckhoff's terminology, ADS refers to the physical aspect of TwinCAT devices, while AMS is for the network side. In this project, the above two terms are used interchangeably. The implemented TwinCAT devices are often referred to as ADS/AMS nodes/devices.

Fig. 44 illustrates components in an AMS message that carries ADS command(s). Specifically, atop TCP, an AMS message contains three parts: AMS/TCP header, AMS header, and ADS data/command(s). In an AMS header, the ADS/AMS connection information is provided along with an ADS command ID pointing to the contained data section. Table 4 provides the list of available ADS commands. For ADS commands that involve 


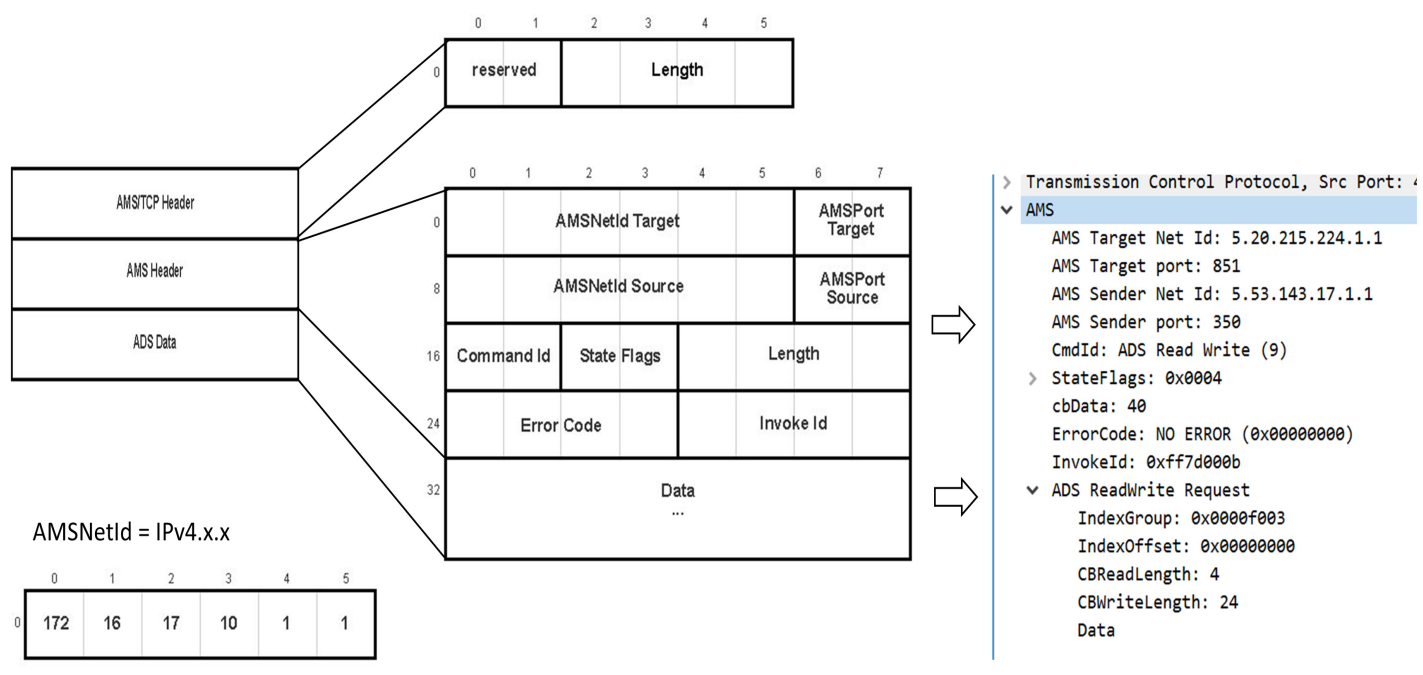

Fig. 44. ADS packet frame (partially reprinted from [28, TCP/IP Reference])

Table 4. ADS Commands (reprinted from [29])

\begin{tabular}{|l|l|l|}
\hline Command ID & Command & Description \\
\hline $0 \times 0000$ & N/A & N/A \\
\hline $0 \times 0001$ & ADS Read Device Info & $\begin{array}{l}\text { Reads the name and the version } \\
\text { number of the ADS device. }\end{array}$ \\
\hline $0 \times 0003$ & ADS Write & $\begin{array}{l}\text { With ADS Read, data can be read } \\
\text { from an ADS device. }\end{array}$ \\
\hline $0 \times 0004$ & ADS Read State & $\begin{array}{l}\text { With ADS Write, data can be } \\
\text { written to an ADS device. }\end{array}$ \\
\hline $0 \times 0005$ & ADS Write Control & $\begin{array}{l}\text { Changes the ADS status and } \\
\text { the device status of an ADS device. }\end{array}$ \\
\hline $0 \times 0006$ & $\begin{array}{l}\text { ADS Add Device } \\
\text { Notification }\end{array}$ & $\begin{array}{l}\text { A notification is created in an } \\
\text { ADS device. }\end{array}$ \\
\hline $0 \times 0007$ & $\begin{array}{l}\text { ADS Delete } \\
\text { Device Notification }\end{array}$ & $\begin{array}{l}\text { It is created before the defined } \\
\text { notification is deleted in an ADS device. }\end{array}$ \\
\hline $0 x 0008$ & $\begin{array}{l}\text { ADS Device } \\
\text { Notification }\end{array}$ & $\begin{array}{l}\text { Data will carry forward independently } \\
\text { from an ADS device to a Client }\end{array}$ \\
\hline $0 \times 0009$ & ADS Read Write & $\begin{array}{l}\text { With ADS Read Write, data will be } \\
\text { written to an ADS device. Additionally, } \\
\text { data can be read from the ADS device. }\end{array}$ \\
\hline
\end{tabular}


process variable operations, e.g., read or write, the target data address of the device is required. The ADS/AMS protocol allows PLC applications to use the variable name to obtain the handle of a remote register so that the device can transmit process information without the knowledge of memory allocation in remote nodes. This feature facilitates the modular design that is focused on nodal functions in the process and encourages individual devices to manage their local memory in a distributed way, which agrees with the dynamics and diversity of industrial applications. In our testbed, we also manage ADS/AMS communications in such a way.
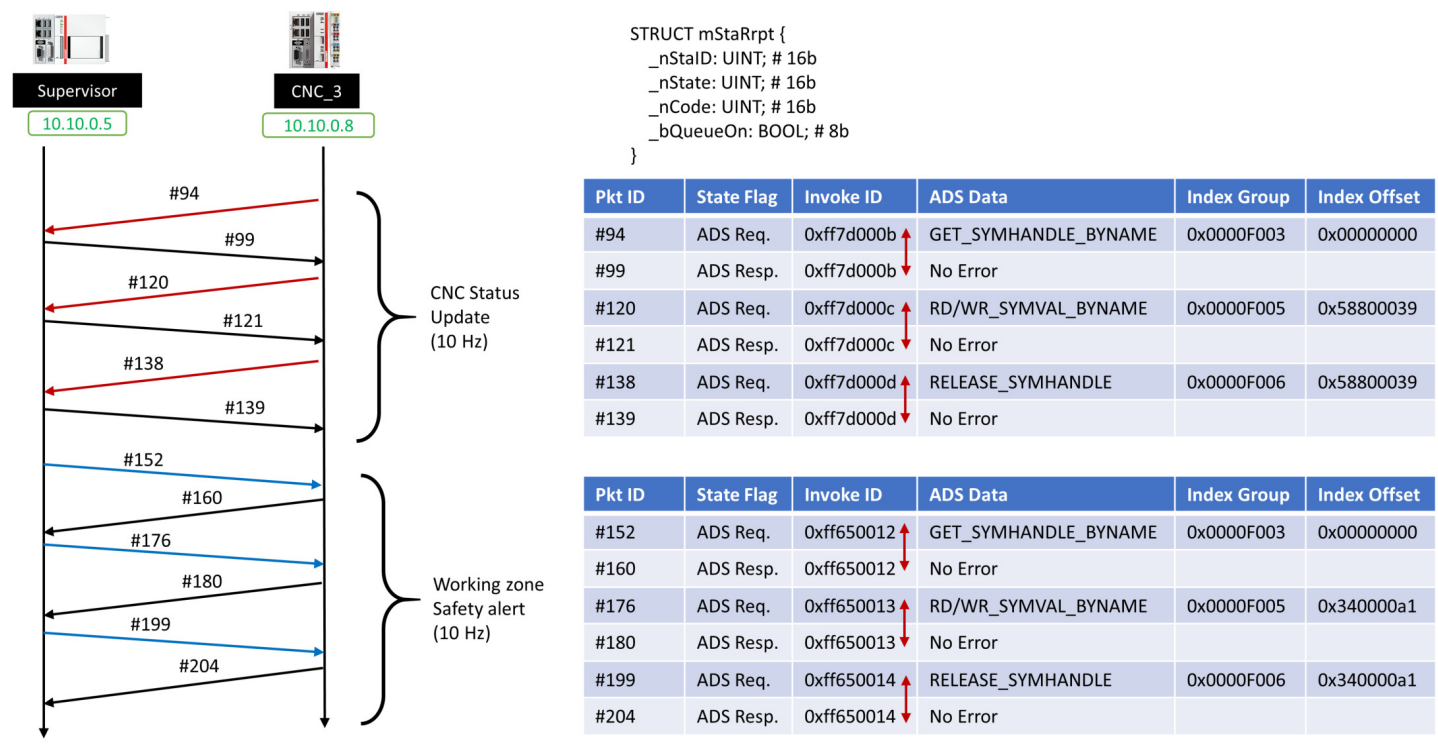

Fig. 45. Handshakes of ADS message transactions in a WireShark trace file

In Fig. 45, two examples of ADS/AMS transactions between testbed PLCs are demonstrated. In a complete transaction, three rounds of handshakes are performed: 1) getting the symbol handle in the remote device by the name, 2) operating the remote symbol using the returned handle, and 3) releasing the handle. In each round, a request-response conversation forms a two-way communication where the request and response are paired with a unique 32-bit Invoke ID in their AMS headers. In the first example, a CNC status report is initiated by the CNC PLC. In the status report, an array of machine status is updated at the supervisor as a data structure known to both devices. In the second example, the supervisor sets a one-bit flag in the CNC to notify the clearance of safety alert regarding a moving robot in the CNC's working space.

We refer the interested readers to the online Beckhoff Information System for further details of ADS/AMS communications [29]. 


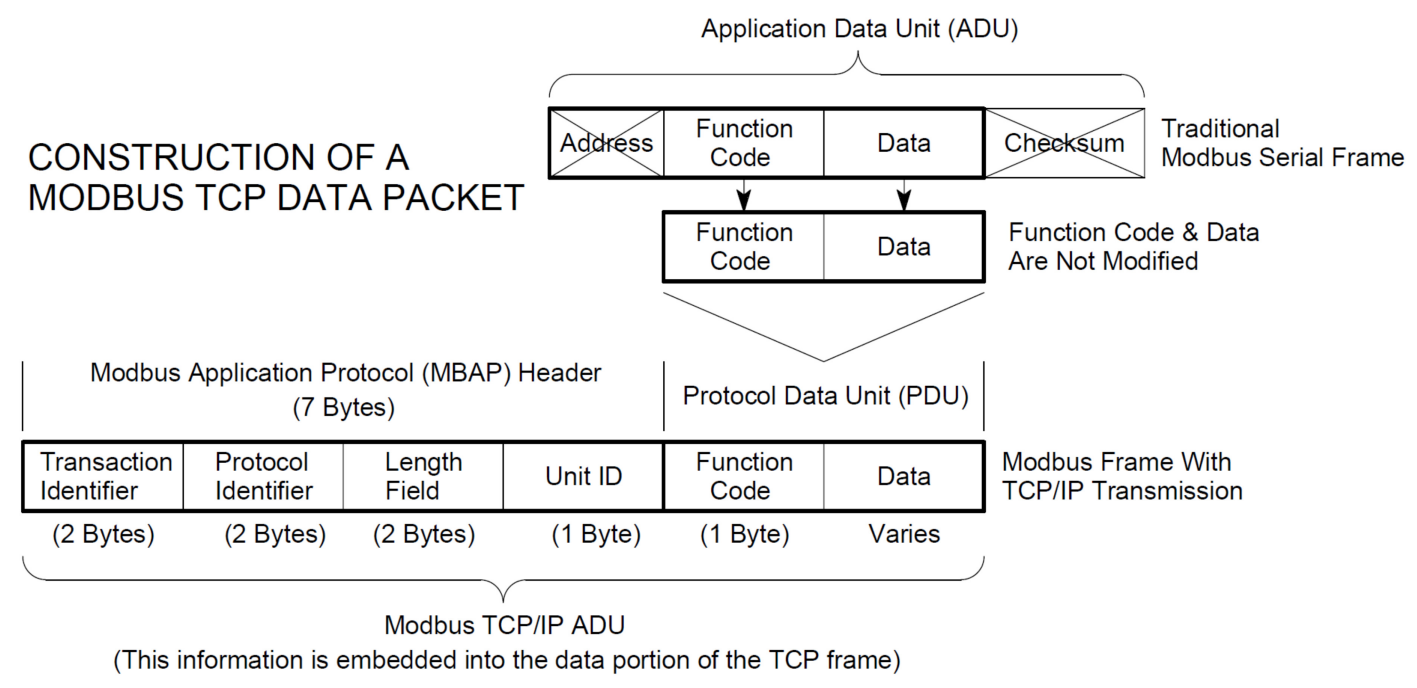

Fig. 46. Construction of a Modbus TCP data packet (reprinted from [27])

\section{A.3 Modbus}

The supervisor communicates with robots through Modbus, which allows the data exchange between heterogeneous industrial appliances in the shared registers at the supervisor. UR3 robots use Modbus TCP in their communications. The Modbus TCP protocol modifies the original Modbus serial frame and adds new header information atop TCP. As shown in Fig. 46, a Modbus TCP packet includes a Modbus Application Protocol (MBAP) header and the Protocol Data Unit (PDU), which contains the tailored Modbus data.

For different register types in Modbus, process variables are accessed through different register address groups as shown in Fig. 47. In this testbed, we only consider digital registers each of which stores a 16-bit numerical data (binary or decimal) referenced in the 4xxxx segment. Accordingly, the available operations in the supported registers can be found in Fig. 48.

Fig. 49 and Fig. 50 illustrate examples of formatting Modbus function messages for reading and writing the registers, respectively. The source and destination port numbers of the TCP header indicate whether or not the TCP payload contains a MBAP request/response: If the TCP source port is 502, one or more Modbus responses are contained; if the TCP destination port is 502, one or more Modbus requests/requries are contained. Accesses to different Modbus registers can be managed in parallel Modbus transactions, even along the same TCP connection. The 32-bit transaction identifier is used in the MBAP header for transaction pairing between requests and responses. For the query of reading holding registers, the response returns the requested register values. For the query of writing a value into the register, the response just repeats the content in the request to confirm 


\begin{tabular}{|c|l|}
\hline Reference & Description \\
\hline 0xxxx & $\begin{array}{l}\text { Read/Write Discrete Outputs or Coils. A 0x reference } \\
\text { address is used to drive output data to a digital output } \\
\text { channel. }\end{array}$ \\
\hline 1xxxx & $\begin{array}{l}\text { Read Discrete Inputs. The ON/OFF status of a 1x } \\
\text { reference address is controlled by the corresponding } \\
\text { digital input channel. }\end{array}$ \\
\hline $3 x x x x$ & $\begin{array}{l}\text { Read Input Registers. A 3x reference register contains } \\
\text { a 16-bit number received from an external source-e.g. } \\
\text { an analog signal. }\end{array}$ \\
\hline $4 x x x x$ & $\begin{array}{l}\text { Read/Write Output or Holding Registers. A 4x register } \\
\text { is used to store 16-bits of numerical data (binary or } \\
\text { decimal), or to send the data from the CPU to an output } \\
\text { channel. }\end{array}$ \\
\hline
\end{tabular}

Fig. 47. Modbus register addresses (reprinted from [27])

\begin{tabular}{|c|l|c|}
\hline CODE & FUNCTION & REFERENCE \\
\hline $01(01 \mathrm{H})$ & Read Coil (Output) Status & 0xxxx \\
\hline $03(03 \mathrm{H})$ & Read Holding Registers & 4 xxxx \\
\hline $04(04 \mathrm{H})$ & Read Input Registers & $3 x x x x$ \\
\hline $05(05 \mathrm{H})$ & Force Single Coil (Output) & 0xxxx \\
\hline $06(06 \mathrm{H})$ & Preset Single Register & 4 xxxx \\
\hline $15(0 \mathrm{FH})$ & Force Multiple Coils (Outputs) & 0xxxx \\
\hline $16(10 \mathrm{H})$ & Preset Multiple Registers & $4 x x x x$ \\
\hline $17(11 \mathrm{H})$ & Report Slave ID & Hidden \\
\hline
\end{tabular}

Fig. 48. Modbus function codes (reprinted from [27])

- 1 Register: 2 bytes

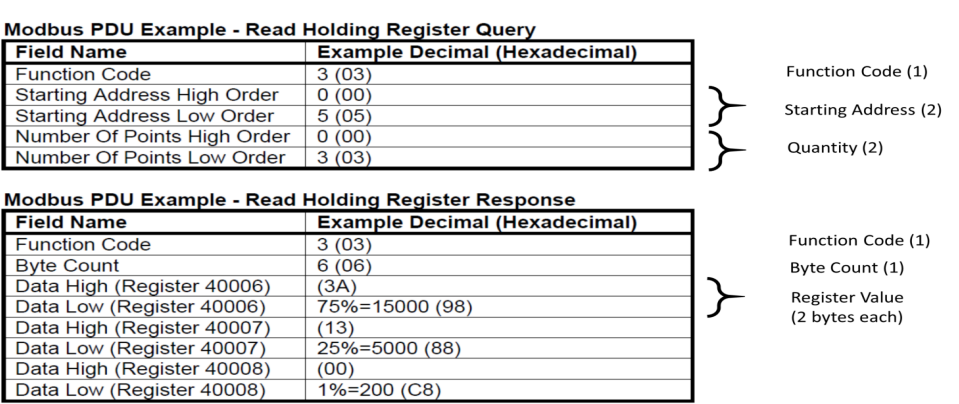

WireShark Dissection Result
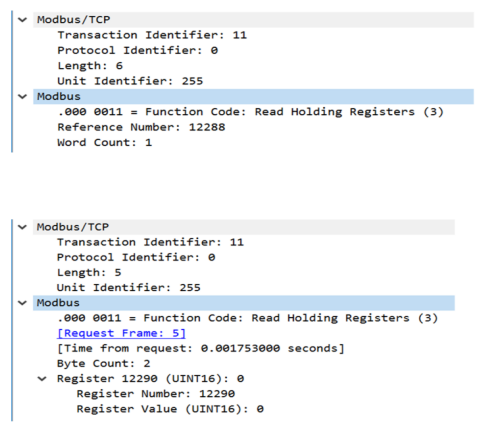

Fig. 49. Modbus function of reading holding registers and capture samples (partially reprinted from [27])

the reception. In the WireShark, we can dissect the transmitted Modbus data and obtain the 
Modbus PDU Example - Preset Holding Register Query and Response

Field Name

Example Decimal (Hexadecimal

\begin{tabular}{|l|l|}
\hline Function Code & $6(06)$ \\
\hline Regster Addrss Hign Order & $0(00)$ \\
\hline
\end{tabular}

Register Address High Order $0(00)$

Register Address Low Order $1(01)$

$\begin{array}{ll}\text { Preset Data High Order } & 0(00)\end{array}$

Preset Data Low Order

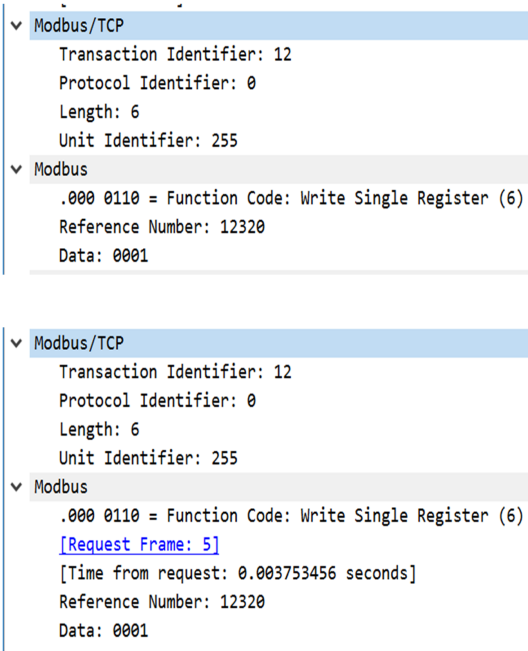

Fig. 50. Modbus function of presetting single register and capture samples (partially reprinted from [27])

useful field information.

We refer the interested readers to the introduction to Modbus TCP/IP for further details of Modbus TCP communications [27]. 


\section{B. Beckhoff PLC Development}

\section{B.1 PLC Data Collection}

Using TwinCAT function libraries, we have developed a routine data collection approach in Beckhoff's PLC programs. In a PLC project, programs are organized as objects, known as Program Organization Units (POUs), which include programs, functions, and function blocks. Each POU has its own internal variables that are defined at the file beginning. Meanwhile, for variables that are shared by multiple POUs, they can be defined and managed in one or separate GVL files which are visible globally in the project. Technically, all global and local variables can be routinely saved into files for measurement purposes. Although it is feasible to record variables every PLC cycle (up to $1 \mathrm{kHz}$ ), it still depends on multiple factors that determine the real update rate, such as subscribed data size, cache capacity, and file open/write speed. In the testbed measurement, the update rate can be selected within a fixed frequency set with the maximum rate at $125 \mathrm{~Hz}$.

\section{B.1.1 Start a PLC Measurement}

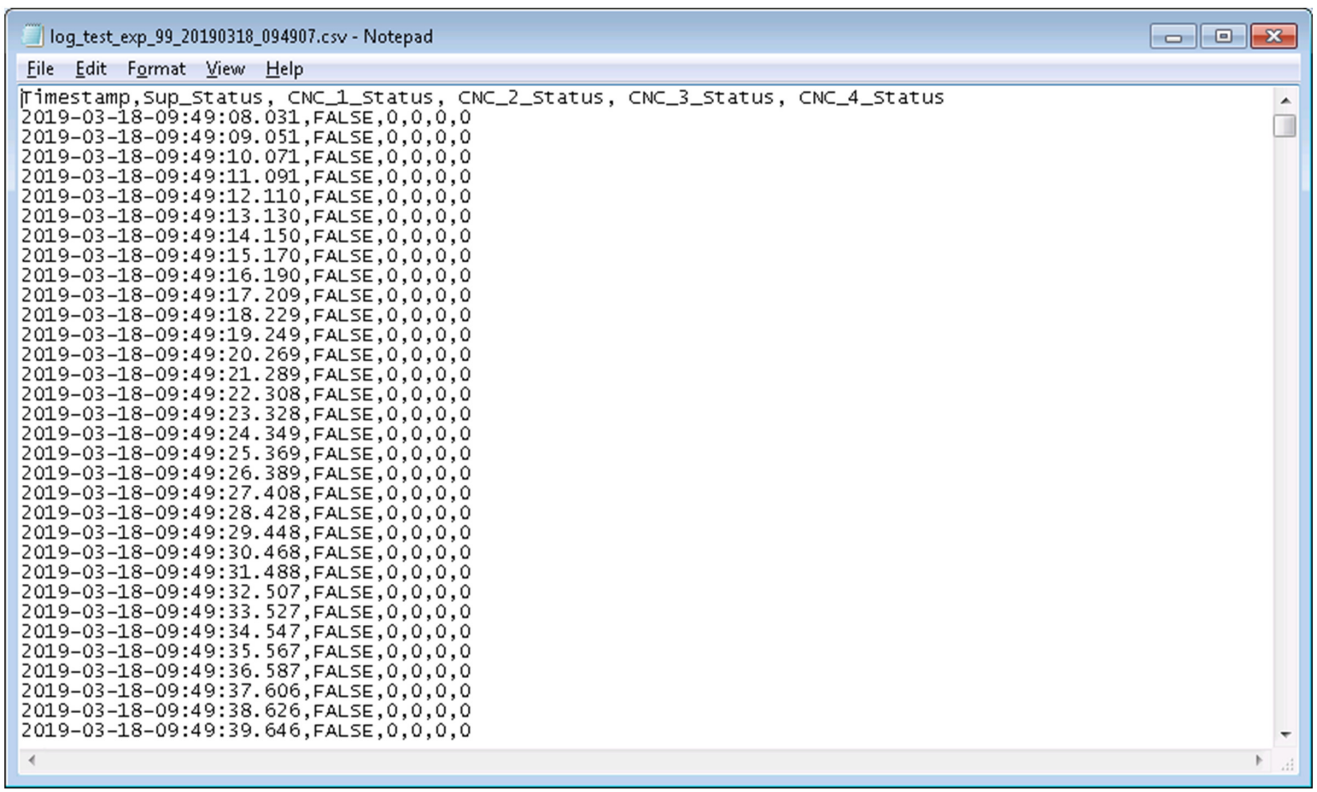

Fig. 51. PLC measurement data in CSV

PLC measurement data are saved as records in CSV format, as shown in Fig. 51. Except the header line on the top, each following row of data contains a list of instant values of the subscribed PLC variables which are separated by comma. The first column is reserved for the record time which is retrieved from the PLC's clock that is synchronized with the testbed's time server via PTPv2. The timing accuracy is on the millisecond level to comply 
with the PLC cycling step (as short as $1 \mathrm{~ms}$ ). Timestamps are later used in analysis to align events from different sources for reconstructing the measured process in the timeline.



Fig. 52. PLC measurement configuration in XML

Measurement configurations are submitted to the PLC through an XML file as shown in Fig. 52. In the file, configuration settings are divided into two main sections: initialization and variable subscription options. The former, labeled in the $\langle$ meas_init $>$ tag, provides global configuration options, such as file naming rules (e.g., indicating start date/time) and data update rate; in the latter, we can enable/disable the collection of available variables as listed in the $\langle$ meas_log_sub $\rangle$ tag. The settings are read by the PLC, stored in the corresponding data structures of the gvMeas GVL, and used by the measurement function Meas_Main in data collection.

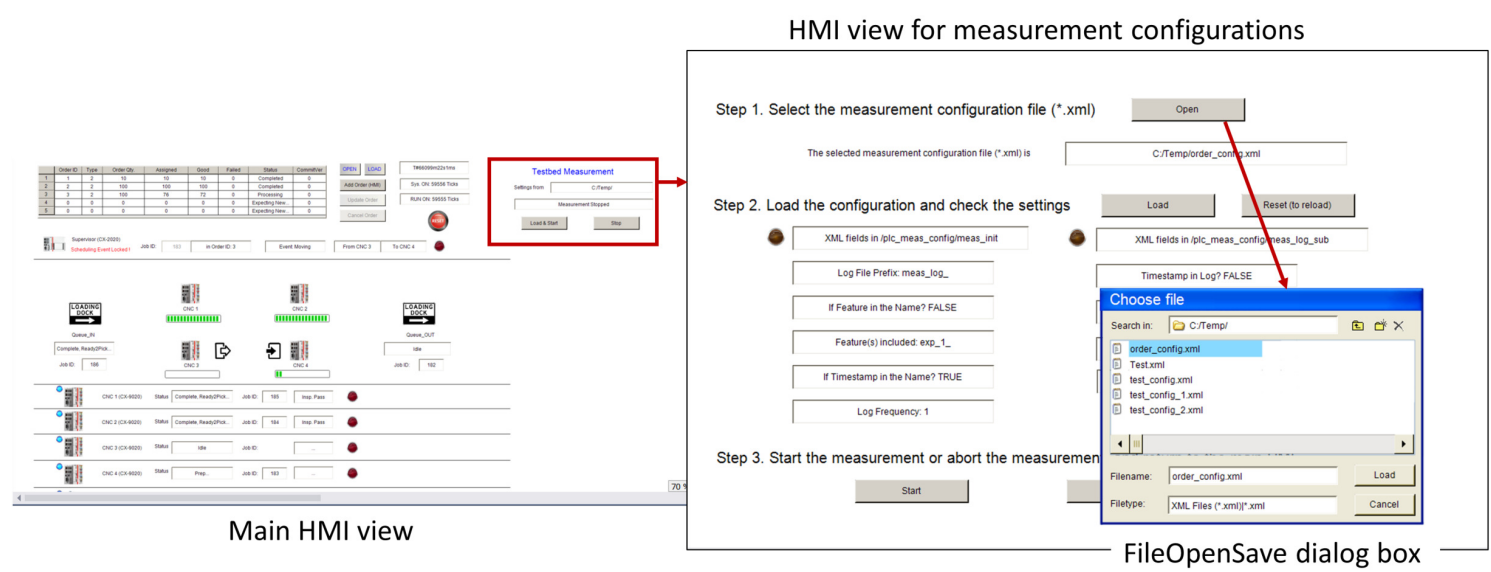

Fig. 53. Load PLC measurement configuration in HMI

We start a new measurement from loading the prepared configuration file. As shown 
in Fig. 53, HMI's main view provides a simple user interface for measurement control. By clicking the "Load \& Start" button, HMI will switch to the view for measurement configuration. There are three steps here.

\section{- Step 1. Select the measurement configuration file}

Click the "Open" button to show the file selection dialog box; Select the configuration file (*.xml) and click "Load";

\section{- Step 2. Load the configuration}

Click the "Load" button. Check the loaded configuration settings shown in the view. If the configuration is not as expected, modify the XML file and repeat Step 1 with the right file. If any error indicator is ON (Errors in reading XML fields), double check XML tags and file format. Reload the correct file starting from Step 1, or click "Reset" and then "Load" to reload the opened file after modifications.

\section{- Step 3. Start the measurement}

If everything looks good in the loaded configuration, click the "Start" button to start the measurement and return to the main HMI view. The measurement setup can be aborted anytime by clicking the "Abort" button to cancel and return to the main HMI view. The measurement status shows the current log file name and location in PLC.

In the main HMI view, the ongoing measurement is shown with its status. It can be stopped by clicking the "Stop" button. Repeat the "open-load-start" operations to start a new measurement.

\section{B.1.2 Adding New PLC Variables in the Subscription List}

As shown in Fig. 52, all available PLC variables that can be subscribed in data collection are listed as logging options in the $<$ meas_log_sub $>$ tag of XML configuration. We can enable or disable individual variables in subscription by setting "true" or "false" values, respectively. For variables that have not been listed, we need to first add them into the list visible to measurement. Before showing how to add a new measurable variable, let us first review how measurements are implemented in the PLC.

In the PLC program, all measurement control variables are stored in the gvMeas GVL. Through interactive operations in HMI, visualization events in measurement-related views, such as mouse clicks, will load the configuration information from XML into gvMeas. Once the measurement gets started, PLC will activate data collection based on gvMeas settings. The Meas_Main function is in charge of main data collection tasks which routinely retrieve the subscribed variable values and write them into the CSV file.

In order to add a new variable into the subscription list, we need to following three steps that prepare PLC for the new measurement option.

- Step 1. Locate the to-be-subscribed variable(s) in the Supervisor, usually defined in GVLs 


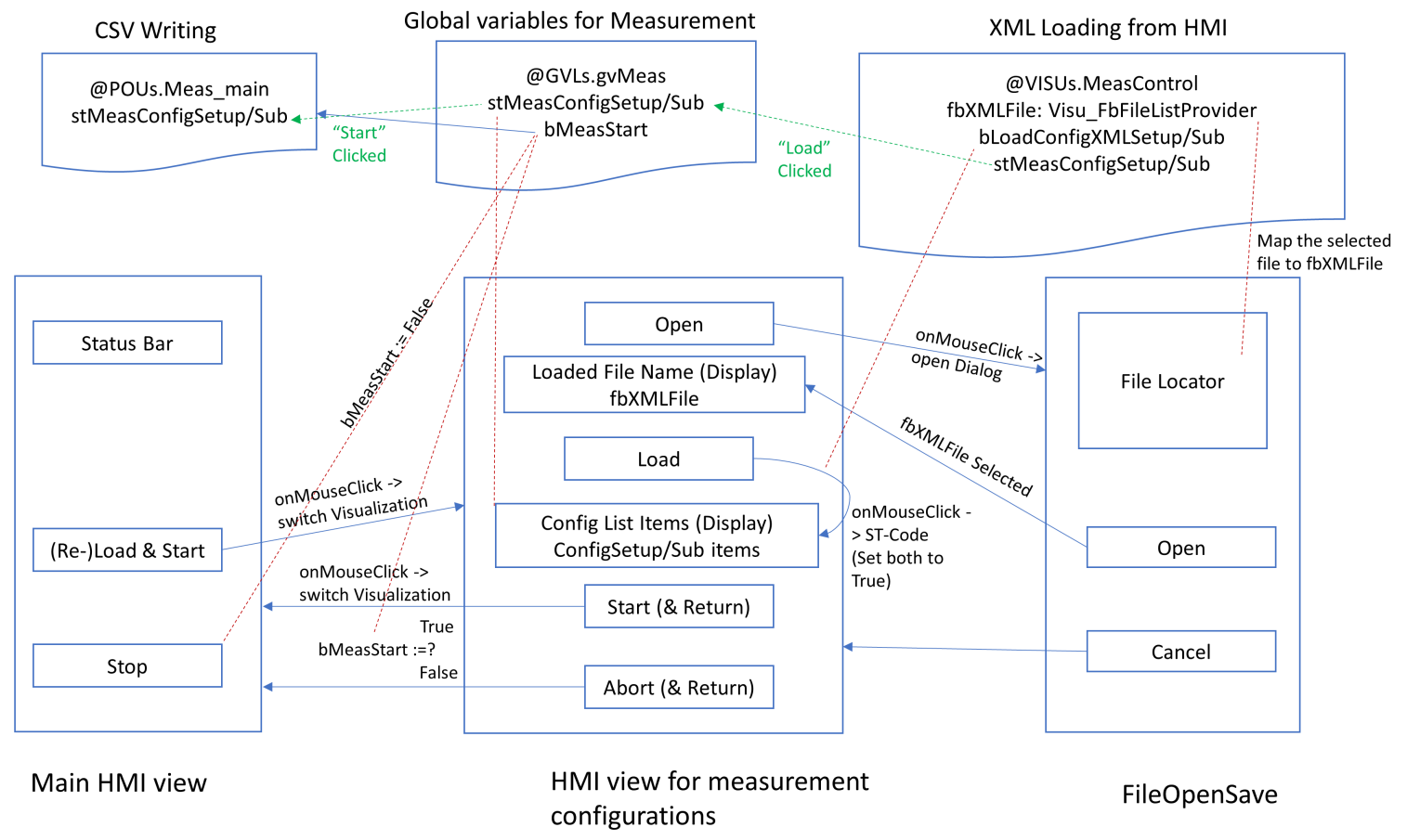

Fig. 54. The design of PLC measurement

\section{- Step 2. Register the variable(s) in PLC (hard coded)}

- Step 2-1. Add selection indicator(s) In the DUTs/MEAS LOG_SUB structure, add BOOL indicators, one for each newly added variable, e.g., _bSupDevTemper with default FALSE.

- Step 2-2. (Optional) Visualize new indicator(s) in HMI To check if the newly subscribed variables are loaded in the runtime, add text field(s) in the measurement configuration view of HMI controlled by VISUs/Visualization_MeasConfig. The text variables (STRING) are mapped from the corresponding variable elements that are newly added into the structure object gvMeas.stMeasLogSub.

- Step 2-3. Add variable(s) in the measurement function In POUs /Meas_Main, update the generation code regarding the header line and data lines with the newly added variable information. Note that $\mathrm{CNC}$ variables are usually organized in vectors each of which may contain four observable data (from CNC 1-4) or more (as STRUCT). In the CSV file, such variables occupy multiple columns in the header line and data lines as counted by elements.

- Step 2-4. Compile and build the PLC project Rebuild the project and load it to the PLC.

- Step 3. Subscribe the data in measurement 
- Step 3-1. Update logging option in XML In the XML configuration file, in the $<$ plc_meas_config $>$ element, add new logging items, e.g., $<$ bSupDevTemper $>$ true $</$ _bSupDevTemper $>$, where the true values means enabling the collection of this value in the CSV file. Save the XML file.

- Step 3-2. Update measurement settings in the runtime Stop the ongoing measurement (if there is any, the measurement status is shown in the main HMI view). Load the update XML file, check the status on display (option, available with Step 2-2), and start the new measurement.

\section{B.2 PLC Time Synchronization}

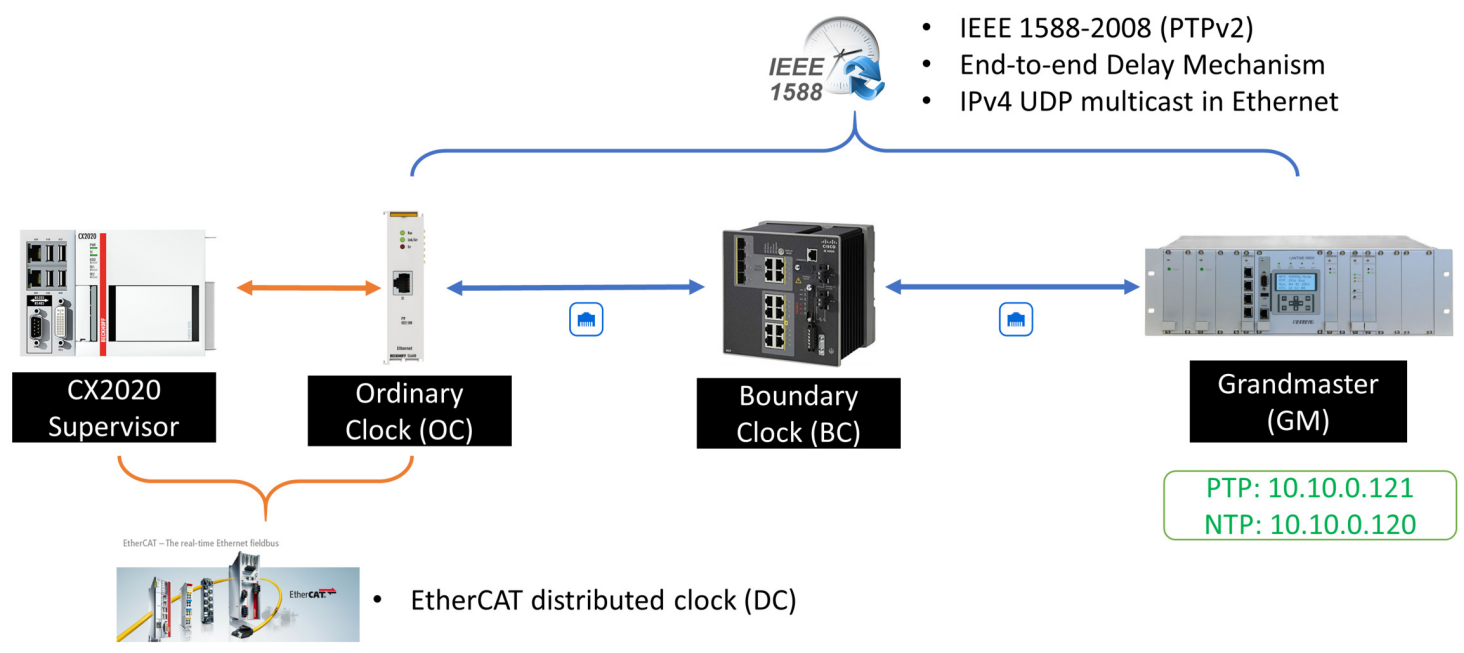

Fig. 55. Time synchronization between PLC and time server through PTPv2

Beckhoff's TwinCAT devices support PTP through an add-on module, the EL6688 IEEE 1588 module. This terminal unit can filter PTP synchronization messages received at the built-in Ethernet port, synchronize its hardware clock to the selected PTP time server, and serve as an OC for providing the synced reference time to PLC applications. Fig. 55 illustrates the end-to-end time synchronization path from the time server to the PLC task cycle. Within the TwinCAT system, different modules exchange timing information through EtherCAT links.

In the network, the synchronization is performed using the end-to-end delay mechanism to calculate the OC's offset from the grand master. Fig. 56 illustrates a round of handshakes that enables the OC to routinely update its clock offset to the selected time server.

The synced OC at EL6688 is also called an EtherCAT Distributed Clock (DC) in Beckhoff systems. DC's offset to the (external) time server clock, DcToExtTimeOffset, is in nanosecond. Serving as the local time reference for the PLC, EL6688 also has to keep measuring the time offset between DC and the PLC's task clock (TC) and maintain it as 
- IEEE 1588-2008 (PTPv2)

- End-to-end Delay Mechanism

- offset $=\frac{\left[\left(t_{2}-t_{1}\right)-\left(t_{4}-t_{3}\right)\right]}{2}$

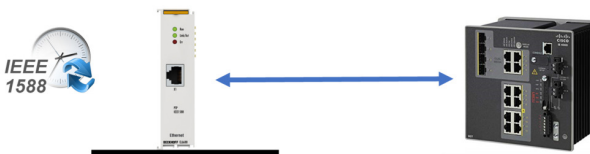

Ordinary

Clock (OC)

Boundary

Clock (BC)

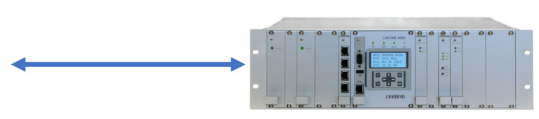

4

What OC

knows

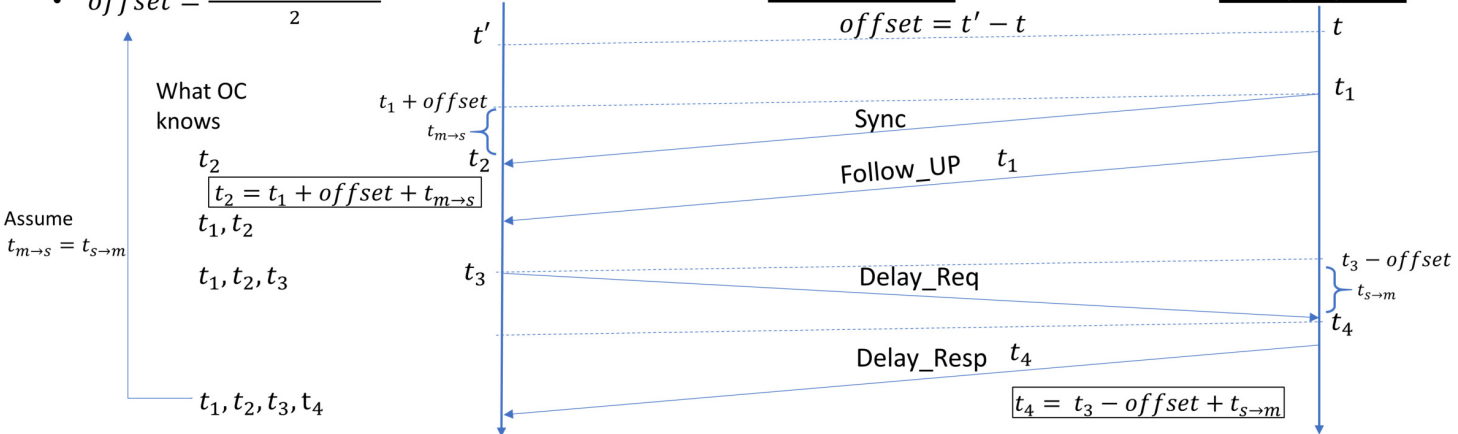

offset $=t^{\prime}-t$

Grandmaste

(GM)

Fig. 56. PTP end-to-end delay mechanism
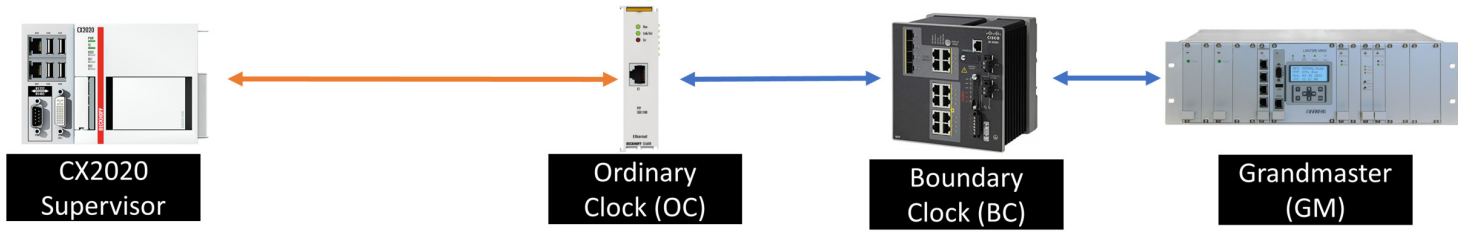

Clock (OC)

Clock (BC)

(GM)

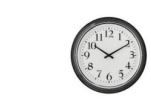

DcToTcTimeOffset

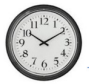

DcToExtTimeOffset

Task clock time

$t_{T C}$

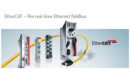

DC time

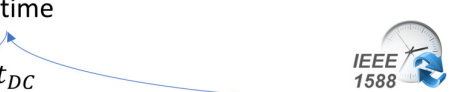

Global time

Real-time (between any two consecutive PTPV2 synchronization handshakes)

PTPv2 Delay Mechanisms (peer-to-peer, end-to-end; 1-step or 2-step) @ $<2 \mathrm{~Hz}$

$t_{T C}^{U T C}=$ GetCurDcTaskTime ()$+$ DcToTcTimeOffset + leapSeconds + DcToExtTimeOffset

Fig. 57. Distributed clock adjustment in PTP time synchronization

another output variable DcToTcTimeOffset in nanosecond. Fig. 57 illustrates how the PLC uses the PTP-synced clock time. When an application calls for the current TC time, e.g., rendering a timestamp for the measurement record, it is calculated in Eq.(2) [30].

$t_{T C}^{U T C}=$ GetCurDcTaskTime ()$+$ DcToTcTimeOffset + leapSeconds + DcToExtTimeOffset

where the GetCurDcTaskTime component is the instant time reading from the system clock at the PLC, the two offset values, i.e., DcToTcTimeOffset and DcToExtTimeOff set, are 
read from the PLC's interface to DC. Note that TwinCAT devices, including EL6688, work internally with the temps atomique international (ATI) time. To obtain the UTC time, it requires a further conversion by adding "leap seconds". In November 2019, there were 37 "leap seconds" [30].

In the saved CSV data, PLC writes the local time information at the beginning of each record row, which also applies the time zone and daylight saving time adjustments to the calculated UTC time. 


\section{Translating Testbed Data to Graph Database}

In Section 7.2, the data importing pipeline has been introduced. This appendix documents more details of individual procedures in pipeline implementation. First, properties of graph elements, i.e., nodes and relationships, are summarized in tables for reference in further data analysis of the graph.

\section{C.1 Graph Nodes, Relationships, and Their Properties}

Nodes, relationships, and their properties used by the testbed GDB are summarized in the following tables. Each property comes with the name and corresponding variable type. As raw data are stored in CSV files as strings, it refers to these tables in converting CSV records to correct variable types, e.g., integer or float, when creating property instances.

\section{C.1.1 Nodes}

Table 5. Nodes and their property tags in the graph for static objects

\begin{tabular}{|c|c|c|c|c|}
\hline Node Label & Property & Type & Value & Note \\
\hline \multirow{3}{*}{ (:Actor) } & name & String & $\begin{array}{l}\{\text { “Supervisor”, "CNC-x”, "Operator”, } \\
\text { "Inspector" }\}\end{array}$ & \\
\hline & type & String & $\{$ \{PLC", "Robot Controller" $\}$ & \\
\hline & model & String & \{“CX2020”, “CX9020”, “UR3”\} & \\
\hline \multirow{3}{*}{$(: N t w k I D)$} & name & String & e.g., "CNC-1_LAN" & \\
\hline & ipAddr & String & "a.b.c.d" where a/b/c/d in $[0,255]$ & IPv4 \\
\hline & host & String & the hosting Actor's name & \\
\hline \multirow{4}{*}{$\begin{array}{l}\text { (:Adapter } \\
{[: \text { Wireless }} \\
: A P /: U E])\end{array}$} & protocol & String & \{“Ethernet", “802.11”\} & \\
\hline & name & String & Port name, e.g., "eth0" & \\
\hline & macAddr & String & "aa:bb:cc:dd:ee:ff" & \\
\hline & host & String & Only for Ethernet NIC, e.g., "Supervisor" & Optional \\
\hline \multirow{4}{*}{ (:Sniffer) } & type & String & $\{"$ wireless" $\}$ & \\
\hline & protocol & String & $\{" 802.11 "\}$ & \\
\hline & name & String & Sniffing port name, e.g., "WLS1" & \\
\hline & macAddr & String & "aa:bb:cc:dd:ee:ff" & \\
\hline
\end{tabular}

As introduced in Section 7.1, a number of static graph nodes are defined in the graph to represent testbed components and their settings in experiments. Table 5 summarizes these node types and their properties used in the graph. Specifically, static nodes include Actor, NtwkID, Adapter (and subcategorical nodes), and Sniffer, which are first created in the graph and serve as anchor points for later added nodes.

- Actor Actor nodes are testbed components which are networked to take industrial operations. In the testbed, Actor nodes include the supervisor PLC, CNC controllers, 
and robotic controllers. In the measurement, we collect network traffic from TAP devices that are collocated with these Actors.

- NtwkID An Actor can also be associated with one or more NtwkID nodes, which contain the network configuration information about the linked Actor. They are also created in the initiation phase and linked with the served Actor nodes.

- Adaptor To connect to the testbed network, each Actor employs one or more adapters. An adapter is a network interface through an Ethernet or wireless link. It can have additional labels to further specify its role in the network. For example, a Wi-Fi router can be represented by a node with the label (:Adapter:Wireless:AP); for WiFi clients in the network, we can use the label (:Adapter:Wireless:UE). Besides, (:Adapter:Ethernet) is used to label the embedded Ethernet interfaces of industrial appliances.

- Sniffer Sniffer is a type of device that captures packets in the select medium for online/offline analysis of communication traffic. Currently we use an Intel NUC as the wireless sniffer to monitor the link quality in the selected $2.4 \mathrm{GHz}$ WLAN channel during the testbed operation.

Dynamic nodes denote testbed records that capture physical process status and network traffic in the work-cell. In current GDB, such nodes include messages, transactions, and physical actions. Table 6 summarizes graph nodes that represent communication messages and associated properties. Each Message node is created based on a single network packet transmitted between industrial devices in the wired/wireless communication link. Packets in the testbed are mainly of the TCP/IP type. Message properties include both the embedded information in PCAP records and necessary meta data to establish relationships.

Transaction nodes refer to operational transactions between industrial devices to coordinate them in work-cell operations. They can be viewed as the abstract of real network packets in the application layer. Message nodes and Transaction nodes have the $n: n$ relationship, i.e., one packet may support multiple transactions (e.g., with aggregated Modbus messages), or one transaction involves multiple packets (e.g., ADS/AMS handshakes). 
Table 6. (:Message) nodes and their property tags in the graph

\begin{tabular}{|c|c|c|c|c|}
\hline Field & Property & Type & Value & Note \\
\hline \multirow{2}{*}{ MAC } & macSrc & String & Source address, "aa:bb:cc:dd:ee:ff" & \\
\hline & macDst & String & Destination address, "aa:bb:cc:dd:ee:ff" & \\
\hline \multirow{4}{*}{ IP } & ipSrc & String & "a.b.c.d" where a/b/c/d in $[0,255]$ & IPv4 \\
\hline & ipDst & String & "a.b.c.d" where a/b/c/d in $[0,255]$ & IPv4 \\
\hline & ipLen & Integer & IP packet length (including IP header) & Bytes \\
\hline & ipChksum & String & "Ox0000abcd" & Optional \\
\hline \multirow{5}{*}{ TCP } & tcpPrtSrc & Integer & TCP source port number & \\
\hline & tcpPrtDst & Integer & TCP destination port number & \\
\hline & tcpSeqNum & String & Relative sequence number (in a PCAP) & Optional \\
\hline & tcpAckNum & String & Relative acknowledgement number & Optional \\
\hline & tcpChksum & String & "0x0000abcd" & Optional \\
\hline \multirow{10}{*}{ Appl. } & adsCmdId & Integer & applicable when proto="ADS" & Optional \\
\hline & adsInvokeId & String & "0xabcd1234", applicable when proto="ADS" & Optional \\
\hline & adsIndexGroup & String & "0x0000abcd", applicable when proto="ADS" & Optional \\
\hline & adsIndexOffset & String & "Oxabcd1234", applicable when proto="ADS" & Optional \\
\hline & adsMatchCode & String & Calculated based on selected ADS features & Optional \\
\hline & mbTransId & Integer & applicable when proto="Modbus" & Optional \\
\hline & mbFuncCode & Integer & applicable when proto="Modbus" & Optional \\
\hline & mbRefNum & Integer & Modbus register number in decimal & Optional \\
\hline & mbRegVal & String & Value in the Modbus register number & Optional \\
\hline & mbMatchCode & String & Calculated based on selected Modbus features & Optional \\
\hline \multirow{13}{*}{ Meta } & msgId & Integer & $\begin{array}{l}\text { unique in a measurement set, encoded } \\
\text { as a 15-digit integer as "1nnttrrxxxxxxxx". }\end{array}$ & Global \\
\hline & copyType & String & By the record source, in $\{$ "tx", "rx" $\}$ & \\
\hline & proto & String & \{“Ether", “TCP", “UDP”, “ADS”, “Modbus"\} & \\
\hline & transRole & String & $\begin{array}{l}\text { The role in a transaction, in }\{\text { "request", } \\
\text { "response", "sole"\} }\end{array}$ & \\
\hline & rsp2MsgId & Integer & $\begin{array}{l}\text { the global msgId of the request message } \\
\text { which is responded to, } 15 \text {-digit }\end{array}$ & $\begin{array}{l}\text { only for } \\
\text { response }\end{array}$ \\
\hline & txTime & Float & the epoch time of Message captured at TX & \\
\hline & rxTime & Float & the epoch time of Message captured at RX & \\
\hline & rxGlobalMsgId & Integer & the global msgId of the RX copy, 15-digit & $\begin{array}{l}\text { only for } \\
\text { TX copy }\end{array}$ \\
\hline & msgCopy & Integer & $\begin{array}{l}\text { for multiple Application messages in the same } \\
\text { TCP payload, copies share the same field } \\
\text { contents of TCP and lower layers }\end{array}$ & \\
\hline & cmdType & String & Industrial application command type & \\
\hline & cmdDetail & String & Industrial application command detail & \\
\hline & tcpRetxMsgId & Integer & $\begin{array}{l}\text { the global msgId of the Message indicated by } \\
\text { "tcp_ana_rto_frame" field in PCAP }\end{array}$ & \\
\hline & $\begin{array}{l}\text { tcpDuplicate } \\
\text {-AckMsgId }\end{array}$ & Integer & $\begin{array}{l}\text { the global msgId of the Message indicated by } \\
\text { "tcp_ana_duplicate_ack_frame" field in PCAP }\end{array}$ & \\
\hline
\end{tabular}


Table 7. (:Transaction) nodes and their property tags in the graph

\begin{tabular}{|c|c|c|c|}
\hline Property & Type & Value & Note \\
\hline proto & String & \{“Ether", “TCP”, “UDP”, “ADS”, “Modbus”\} & \\
\hline reqTxId & Integer & $\begin{array}{l}\text { the msgId of the init Message (Request) } \\
\text { recorded at TX }\end{array}$ & \\
\hline rspRxId & Integer & $\begin{array}{l}\text { the 15-digit global Message ID copied from } \\
\text { rxGlobalMsgId of the closing Message (Response) }\end{array}$ & Optional \\
\hline timeStart & Float & the txTime of the init Message (Request) node & \\
\hline timeStop & Float & the rxTime of the closing Message (Response) & Optional \\
\hline status & String & $\begin{array}{l}\{\text { “Open"(init), "Closed" }\}, \text { switched to } \\
\text { "Closed" once all relationships are created }\end{array}$ & \\
\hline cmdType & String & Industrial application command type & \\
\hline cmdDetail & String & Industrial application command detail & \\
\hline transCopy & String & $\begin{array}{l}\text { copied from msgCopy of the Request Message, } \\
\text { used in Modbus transactions }\end{array}$ & Optional \\
\hline mbTransId & Integer & $\begin{array}{l}\text { copied from mbTransId of the Request, } \\
\text { used in Modbus transactions }\end{array}$ & Optional \\
\hline
\end{tabular}

Table 7 summarizes node properties regarding a Transaction node. Each Transaction serves a specific industrial action purpose in processing monitoring and control. Transactions in the testbed include ADS/AMS messages (between the supervisor and CNCs) and Modbus messages (between the supervisor and robotic controllers). 
Table 8. Physical action nodes and their property tags in the graph

\begin{tabular}{|c|c|c|c|c|}
\hline Node & Property & Type & Value & Note \\
\hline \multirow{3}{*}{ (:PhyAction) } & action & String & description to the action & \\
\hline & timeStart & Float & The action start time in the epoch time & \\
\hline & timeStop & Float & The action stop time in the epoch time & \\
\hline \multirow{8}{*}{ (:URSchedule) } & actor & String & the Actor's name, i.e., "Supervisor" & \\
\hline & stateId & Integer & $\begin{array}{l}\text { unique type index associated with the } \\
\text { Actor (in case an Actor may have } \\
\text { multiple states to collect) }\end{array}$ & \\
\hline & actionId & String & $\begin{array}{l}\text { unique in the measurement set; } \\
\text { " } 3 \text { " +actorId(2)+stateId(1)+index }(8)\end{array}$ & 14-digit \\
\hline & time & Float & The epoch time when the state begins & \\
\hline & target & String & $\begin{array}{l}\text { The name of the scheduled Actor, e.g., } \\
\text { "Inspector" }\end{array}$ & \\
\hline & targetAction & String & $\begin{array}{l}\text { The action assigned to the target, e.g., } \\
\text { "inspect" }\end{array}$ & \\
\hline & waypointId1 & Integer & $\begin{array}{l}\text { The index of job stop } 1 . \text { For OPT, it is } \\
\text { the part pick-up location; for INS, it is } \\
\text { the inspection location. }\end{array}$ & \\
\hline & waypointId2 & Integer & $\begin{array}{l}\text { The index of job stop } 2 . \text { For OPT, it is } \\
\text { the part drop-off location; for INS, it } \\
\text { repeats the set value of waypointId } 1 \text {. }\end{array}$ & \\
\hline \multirow{6}{*}{ (:SensorState) } & actor & String & the host actor's name & \\
\hline & stateId & Integer & $\begin{array}{l}\text { unique type index associated with the } \\
\text { Actor (in case an Actor may have } \\
\text { multiple states to collect) }\end{array}$ & \\
\hline & sensorType & String & $\begin{array}{l}\text { The sensor type of the state source, } \\
\text { e.g., "Proximity" of the part holder } \\
\text { in an CNC }\end{array}$ & \\
\hline & actionId & String & $\begin{array}{l}\text { unique in the measurement set; } \\
\text { " } 3 \text { "+actorId(2)+stateId(1)+index }(8)\end{array}$ & 14-digit \\
\hline & time & Float & The epoch time when the state begins & \\
\hline & value & Integer & the state value of the sensor output & \\
\hline \multirow{6}{*}{$(:$ RouteState $)$} & actor & String & $\begin{array}{l}\text { The actor robot who is assigned with } \\
\text { this route instruction }\end{array}$ & $\begin{array}{l}\text { By the } \\
\text { scheduler }\end{array}$ \\
\hline & actionId & Integer & $\begin{array}{l}\text { unique in the measurement set; } \\
\text { " } 3 \text { " +actorId(2)+stateId(1)+index }(8)\end{array}$ & 14-digit \\
\hline & time & Float & The epoch time when the route begins & \\
\hline & $\begin{array}{l}\text { optRoute } \\
\text { From }\end{array}$ & Integer & $\begin{array}{l}\text { Job stop index from which the part } \\
\text { is moved by robot }\end{array}$ & $\begin{array}{l}\text { Optional, } \\
\text { OPT only }\end{array}$ \\
\hline & $\begin{array}{l}\text { optRoute } \\
\text { To }\end{array}$ & Integer & $\begin{array}{l}\text { Job stop index to which the part } \\
\text { is moved by robot }\end{array}$ & $\begin{array}{l}\text { Optional, } \\
\text { OPT only }\end{array}$ \\
\hline & inspRoute & Integer & $\begin{array}{l}\text { Job stop index at which the part is } \\
\text { inspected by robot }\end{array}$ & $\begin{array}{l}\text { Optional, } \\
\text { INS only }\end{array}$ \\
\hline
\end{tabular}


Table 8 summarizes physical action (PhyAction) nodes used in the graph database, which are built upon production measurement data. A PhyAction node refers to a record of measured industrial action in work-cell operations. These actions are recorded by external monitors, such as UR3's RTDE interfaces and PLC's recording, other than network sniffers. Since PhyAction nodes are only linked with Actors, we can load PhyAction nodes into the graph in parallel with network-related Message and Transaction nodes.

Table 9. QoSReport nodes and their property tags in the graph

\begin{tabular}{|c|c|c|c|c|}
\hline Node & Property & Type & Value & Note \\
\hline \multirow{11}{*}{ (:QoSReport) } & time & Float & Packet reception time in epoch & \\
\hline & dataRate & Integer & WLAN data rate in Mbps & Radiotap Header \\
\hline & rssi & Integer & $\begin{array}{l}\text { WLAN signal strength indicator, } \\
\text { in } \mathrm{dBm}\end{array}$ & Radiotap Header \\
\hline & channel & Integer & WLAN channel index, $[1,11]$ & Radiotap Header \\
\hline & txAddr & String & MAC address of the packet sender & \\
\hline & rxAddr & String & MAC address of the packet receiver & \\
\hline & rprtId & Integer & $\begin{array}{l}\text { unique in a measurement set, encoded } \\
\text { as " } 2 \text { " }+\operatorname{SnifferId}(2)+\text { index }(8)\end{array}$ & 11-digit \\
\hline & anchorId & Integer & $\begin{array}{l}\text { The pairing index between a Message } \\
\text { and a QoSReport, unique in the } \\
\text { measurement set }\end{array}$ & $\begin{array}{l}\text { To build } \\
\text { [:COVERED] } \\
\text { relationship }\end{array}$ \\
\hline & msgId & Integer & $\begin{array}{l}\text { The global msgId of the Message } \\
\text { that this QoSReport covers }\end{array}$ & $\begin{array}{l}\text { To build } \\
\text { [:COVERED] } \\
\text { relationship }\end{array}$ \\
\hline & $\begin{array}{l}\text { msgCopy } \\
\text { Idx }\end{array}$ & Integer & $\begin{array}{l}\text { The index of the covered Message in } \\
\text { all Messages which are covered by } \\
\text { this QoSReport node }\end{array}$ & $\begin{array}{l}\text { To build } \\
\text { [:COVERED] } \\
\text { relationship }\end{array}$ \\
\hline & msgIpSrc & String & The source IP of the covered Message & $\begin{array}{l}\text { To build } \\
\text { [:COVERED] } \\
\text { relationship }\end{array}$ \\
\hline
\end{tabular}

Table 9 summarizes properties used in the QoSReport node which are obtained from the Radiotap header data of the sniffer's output.

\section{C.1.2 Relationships}

Table 10 summarizes relationships between nodes in the graph. They are created based on conditions that links two nodes given their properties. Note that relationships are directional. 
Table 10. Relationships and their creation conditions in the graph

\begin{tabular}{|c|c|c|c|c|}
\hline Relationship & Connect Node & To Node & Condition(s) & Note \\
\hline TX & (a:Actor) & (m:Message) & $\begin{array}{l}\text { m.ipSrc=n.ipAddr where } \\
\text { (a)-[:HAS]->(n:NtwkID) }\end{array}$ & \\
\hline RX & (a:Actor) & (m:Message) & $\begin{array}{l}\text { m.ipDst=n.ipAddr where } \\
\text { (a)-[:HAS]->(n:NtwkID) }\end{array}$ & \\
\hline \multirow[t]{2}{*}{ SUPPORTED } & \multirow[t]{2}{*}{ (m:Message) } & \multirow[t]{2}{*}{ (t:Transaction) } & $(\mathrm{t})$ is created from $(\mathrm{m})$ & $\begin{array}{l}\text { m.transRole= } \\
\text { 'Request' }\end{array}$ \\
\hline & & & m.rsp2MsgId=t.reqTxId & $\begin{array}{l}\text { m.transRole= } \\
\text { 'Response' }\end{array}$ \\
\hline SCHEDULED & (u:URSchedule) & (r:RouteState) & $\begin{array}{l}\text { u.actionId AND r.actionId } \\
\text { are coupled in timeline }\end{array}$ & $\begin{array}{l}\text { Performed separately } \\
\text { for OPT and INS }\end{array}$ \\
\hline TOOK & (a:Actor) & (p:PhyAction) & a. name $=$ p.actor & $\begin{array}{l}\text { Also apply to } \\
\text { (:URSchedule); } \\
\text { (:RouteState); } \\
\text { (:SensorState) }\end{array}$ \\
\hline FOLLOWED & (a:PhyAction) & (b:PhyAction) & a.actionId=b.actionId-1 & $\begin{array}{l}\text { Also apply to } \\
\text { (:URSchedule); } \\
\text { (:RouteState); } \\
\text { (:SensorState) }\end{array}$ \\
\hline $\begin{array}{l}\text { PARTICIPATED } \\
\text { _IN }\end{array}$ & (a:Actor) & (t:Transaction) & $\begin{array}{l}\text { (a)-[:TX/RX]->(m:Message) } \\
-[: \text { SUPPORTED]- }>\text { (t) }\end{array}$ & \\
\hline \multirow{2}{*}{$\begin{array}{l}\text { CONNECTED } \\
\text { _THROUGH }\end{array}$} & \multirow[t]{2}{*}{ (a:Actor) } & $\begin{array}{l}\text { (n:Adapter } \\
\text { :Ethernet) }\end{array}$ & a. name $=$ b.host & $\begin{array}{l}\text { For built-in Ethernet } \\
\text { interface(s) }\end{array}$ \\
\hline & & $\begin{array}{l}\text { (n:Adapter } \\
\text { :Wireless:AP } \\
\text { /:UE) }\end{array}$ & a.name AND b.macAddr & $\begin{array}{l}\text { Specified in } \\
\text { experiments }\end{array}$ \\
\hline NEXT & (a:PhyAction) & (b:PhyAction) & a.actionId AND b.actionId & $\begin{array}{l}\text { Call Neo4j APOC's } \\
\text { node link function }\end{array}$ \\
\hline COVERED & (q:QoSReport) & (m:Message) & $\begin{array}{l}\text { m.msgCopy=q.msgCopyIdx } \\
\text { AND m.msgId=q.msgId }\end{array}$ & \\
\hline TRIGGERED & (t:Transaction) & (r:RouteState) & $\begin{array}{l}\text { t.reqTxId AND r.actionId } \\
\text { are coupled in timeline }\end{array}$ & $\begin{array}{l}\text { Modbus commands, } \\
\text { performed separately } \\
\text { for OPT and INS }\end{array}$ \\
\hline HAS & (a:Actor) & (n:NtwkID) & a.name $=$ n.host & Static \\
\hline GENERATED & (w:Wireless) & (q:QoSReport) & $(w)->(q)$ & w/ a single Sniffer \\
\hline $\begin{array}{l}\text { COLOCATED } \\
\text { _WITH }\end{array}$ & (w:Wireless) & (a:Actor) & a.name AND w.name & $\begin{array}{l}\text { Specified in } \\
\text { experiments }\end{array}$ \\
\hline
\end{tabular}

\section{C.2 Data Importing Flow}

\section{C.2.1 Overview}

To streamline data processing steps, a software package is developed. Fig. 58 illustrates its main function modules and highlights the data pipeline along which measurement data are converted into graph elements by the graph data model addressed in Section 7.1.1. The code is mainly written in Python 3.6.6, which also employs Neo4j Desktop 3.5.6 as the GDB application. Using the Neo4j bolt driver, the Python script can visit the Neo4j database with the embedded Cypher query statements. In addition, Bash scripts use TShark's functions 


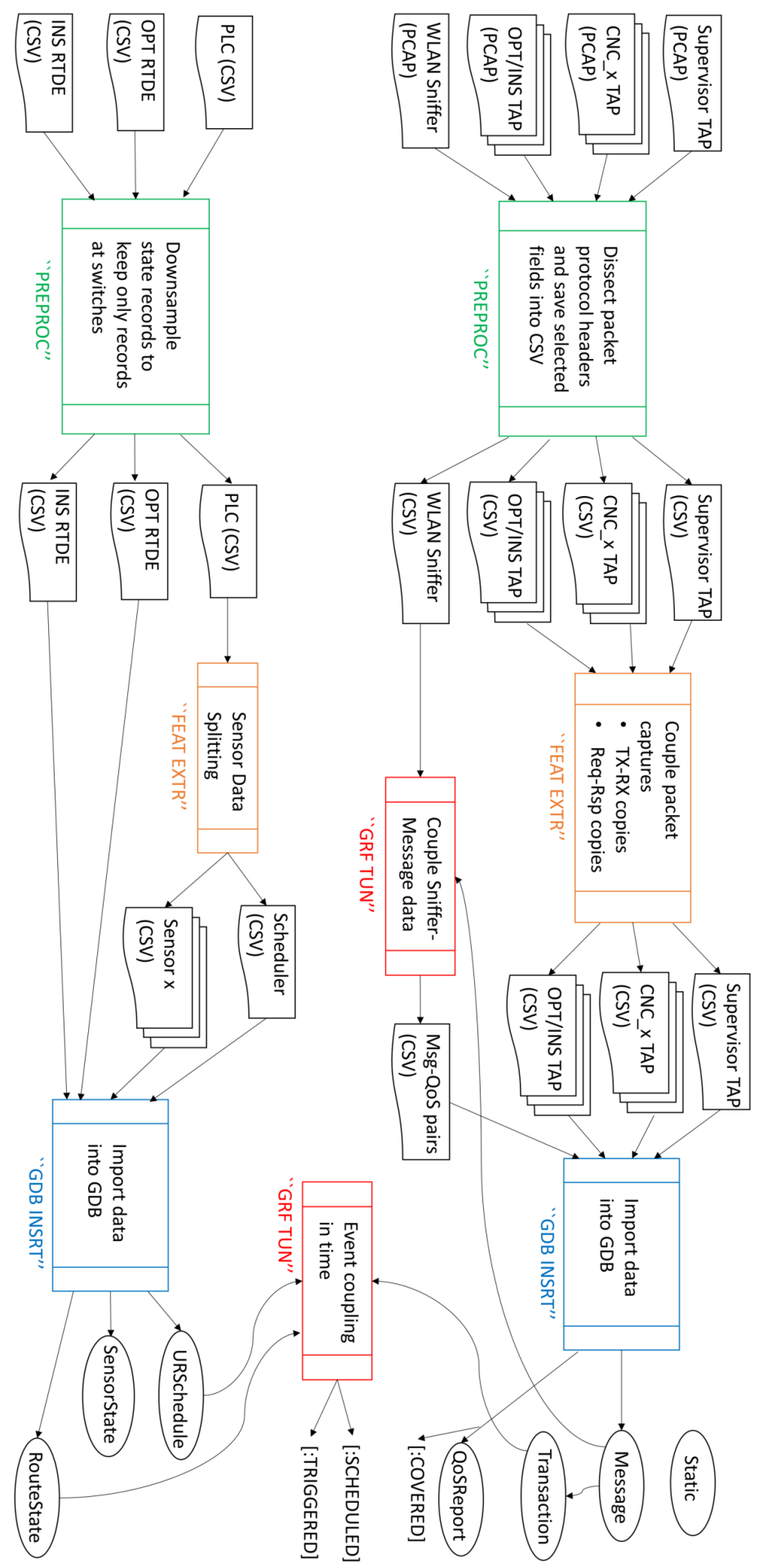

Fig. 58. Implementing the pipeline of importing testbed data into GDB 
to dissect network packet captures for header information of different network protocols.

As shown in Fig. 58, network traffic data and production modules are treated according to their own data features. In the preprocessing (PREPROC) stage, network captures in PCAP files are first processed by a packet dissection tool to extract multiple fields from the encapsulated packet headers and save them into separate columns in CSV files. In this tool, Bash scripts use TShark's protocol libraries to resume header fields from packet bytes. These fields will later serve as either properties of packet-oriented graph nodes, such as Messages and Transactions, or pairing conditions to create relationships showing the communication history. In the next stage, i.e., feature extraction (FEAT EXTR), separate packet copies will be paired according to interconnections in transmissions, such as two copies captured on both ends of a link. These pairing information will also be saved as additional meta data in CSV files and used to build the graph. Next, in graph database insertion (GDB INSRT), rows of CSV files will be imported into the GDB to create nodes and relationships. However, it is not the end of the building process. The graph tuning (GRF TUN) stage takes further actions to create more relationships to link nodes together regarding their explicit and implicit connections in the network. Such operations may run solely within GDB, however, it is more common to utilize intermediate graph information combined with raw data to unveil more insight relationships.

In a similar way, OT data collected from work-cell modules, i.e., PLC and robot data, are treated in parallel. For example, in PREPROC, OT data are first downsampled to filter out constant variable readings and keep records indicating state transitions. Such operations are purposed for cleaning raw data and reducing computing load of following steps. In GRF TUN, different node events are assorted by time so that delays in communication networks and control processes can be studied in analysis.

\section{C.2.2 Packet Dissection}

To treat the packet capture data collected by WireShark instances in the PREPROC stage, we utilize the command-line tool called TShark to dissect captured packets in the raw data files of PCAP and access the packet header information [31]. The content of selected packet header fields will be extracted and saved as CSV data where each row represents the information of one packet capture while columns store the meta and header fields. CSV files are exported to the next step for further process before importing the data into the graph.

\section{C.2.3 Coupling Packet Captures in Communication Links}

In the FEAT EXTR stage, the main task to create relationships between graph nodes. Finding a relationship relies on the engineering knowledge of work-cell operations and network flows to couple discrete event records. Such techniques are widely used in treating our testbed data. As an example, we will showcase how to pair distributed packet copies as well as defining transactions that trigger industrial control actions. 
The coupling can be classified into two different types. Type I is to couple packet records in CSV files based on their relationships in link transmissions and communication protocols; Type II is to link the external add-on data with the existing graph.

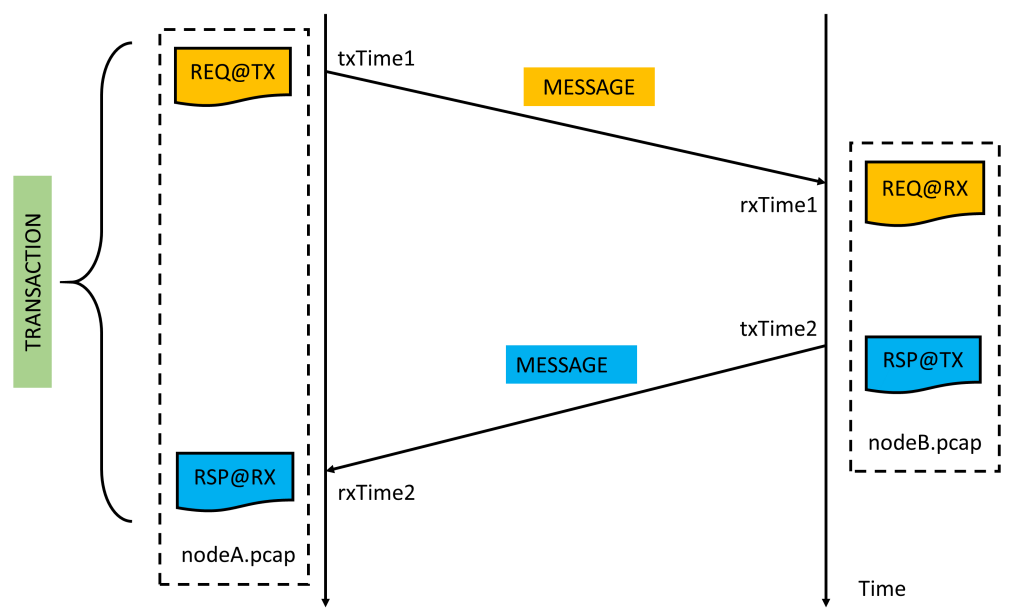

Fig. 59. Timeline illustrations of multiple network captures in a control command transaction

As shown in Fig. 59, we can find the copies of packet captures related with a message transmission in the capture files at both transceivers. As a transaction includes the twoway message exchange, then we have to find four packet captures in order to create two Message nodes and one Transaction node in the graph. The timestamp embedded in each capture record is the main reference information used in the coupling process.



Fig. 60. Graph nodes in a control command transaction 


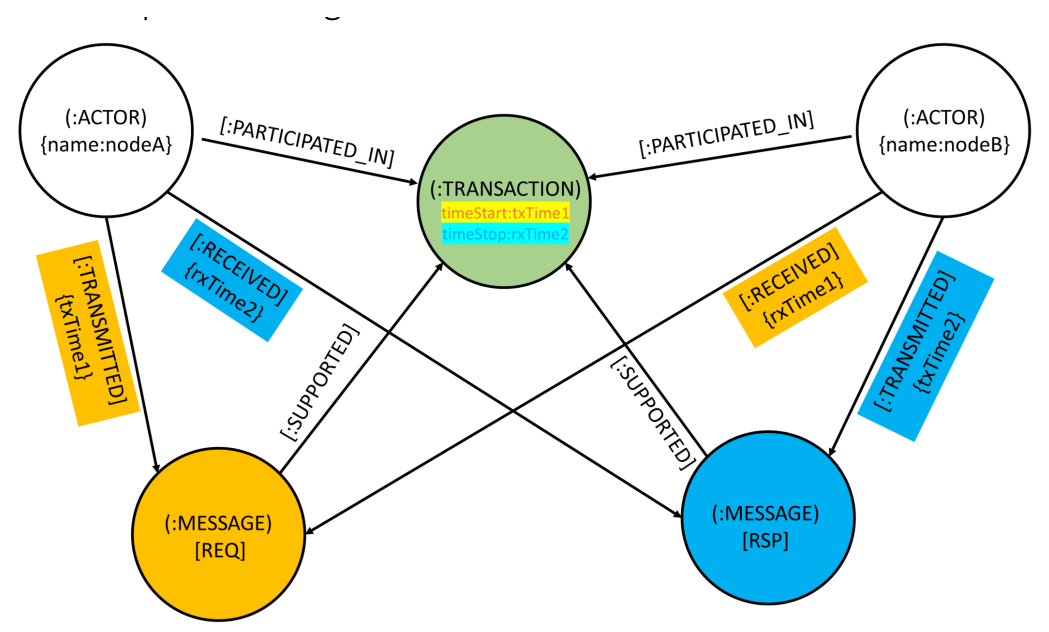

Fig. 61. Graph nodes and the established relationships in a control transaction

Fig. 60 illustrates the graph nodes that are rendered based on the packet captures. Four Message nodes will be created directly from the PCAP data. Accordingly, Message properties will be set by corresponding CSV columns. Note that a Message node is designed to contain the properties related with both the "TX" and "RX" copies of a real message. We will combine the Message copies of the same message, e.g., REQ@TX and REQ@RX, to leave only one node, i.e., REQ@TX. The nodes shown in the dashed circles will be removed from the final graph to reduce the size.

Fig. 61 shows the result of the coupling processing related with a control transaction. Further details at individual steps can be found in Fig. 62.

\section{C.2.4 Cypher Queries and Python-Neo4j Interface}

Cypher is Neo4j's query language that interfaces with the GDB runtime. With a SQLlike syntax, Cypher also provides drivers in common programming languages, such as $\mathrm{C}$, Python, and Java, which use the bolt protocol.

In the GDB-related operations, we use Python 3.6.6 to send Cypher queries to the running graph in Neo4j Desktop 3.5.6 which returns the requested data for further analysis. There are a few available Python packages on the Python-Neo4j interface, such as neo4j and py2neo. Their main difference is the format of embedding Cypher queries in a Python script.

For example, the Python code using ph2neo can be written in the following way.

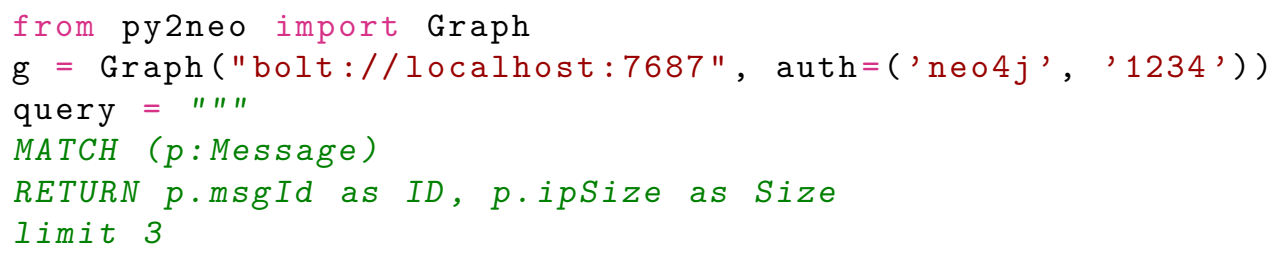



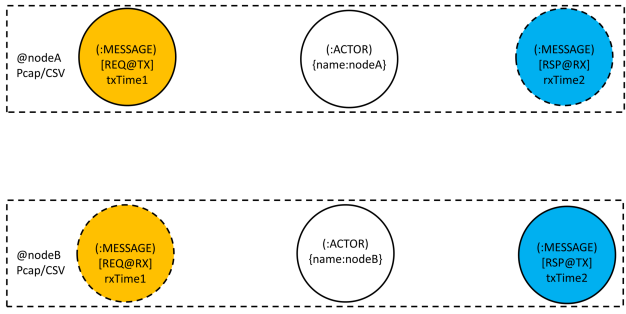

a Nodes imported from packet captures

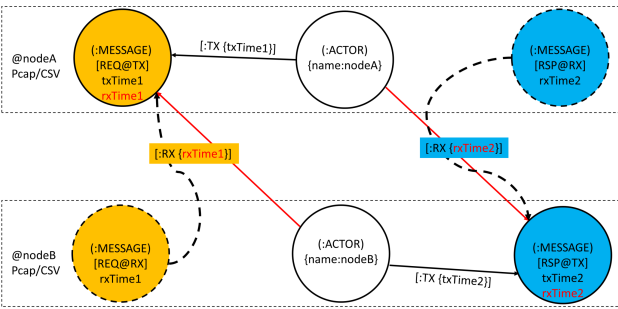

c Build RX relationships

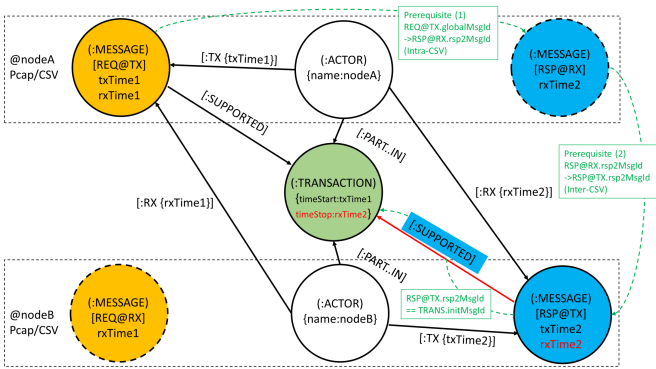

e Set Transaction properties
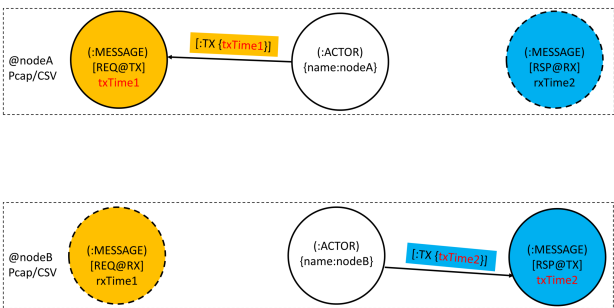

b Build TX relationships

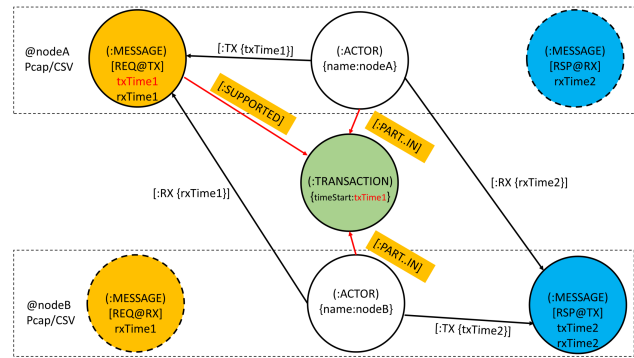

d Create the Transaction node

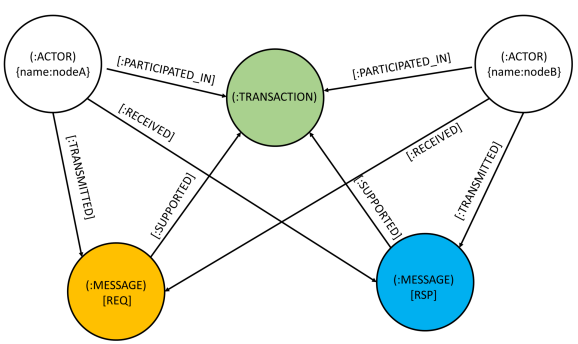

f Final view

Fig. 62. Coupling procedures to pair network captures in a communication transaction.

" " "

$a=g \cdot r u n(q u e r y) \cdot \operatorname{data}() \quad \%$ return the query data as a list of dictionaries

$a=g \cdot r u n(q u e r y) . s t a t s() \quad \%$ return the query statistics

In the above sample code, the query asks for the "msgId" and "ipSize" information of at most 3 Message nodes from the graph. A Neo4j Graph object is first initialized with the graph address and authentication information. The Cypher query is then set as a string variable and sent to the database by the run() function. The data() functions returns the query result as a list of dictionaries. Each dictionary, i.e., one matched Message node out of the returned 3, contains key-value pairs, e.g., the "ID" and "Size" keys which are the alias of the requested properties, respectively. 


\section{Testbed Equipment Specifications}

Table 11. Testbed equipment hardware and software specifications. Numbers in the parentheses denote the quantities of equipment components.

\begin{tabular}{|c|c|c|c|c|}
\hline Equipment & \multicolumn{2}{|r|}{ Hardware } & \multicolumn{2}{|c|}{ Software } \\
\hline \multirow{5}{*}{ Central data collector (1) } & hostname & RIVA & OS & $\begin{array}{l}\text { Ubuntu 18.04.1 } \\
\text { desktop 64-bit }\end{array}$ \\
\hline & $\mathrm{CPU}$ & Intel Xeon E5-2620 2.4 GHz (1) & UR3 TCP/IP client & RTDE Python driver \\
\hline & Memory & 16 GB DDR4 $2400 \mathrm{MHz}(4)$ & $\begin{array}{l}\text { Packet capture \& } \\
\text { measurement }\end{array}$ & TShark \\
\hline & Hard drive & 1 TB SSD (1) & Time sync & linuxptp \\
\hline & Network & Gigabit Ethernet ports (10) & & \\
\hline \multirow{5}{*}{$\begin{array}{l}\text { Data processor \& } \\
\text { GDB server (1) }\end{array}$} & hostname & Fishbone2 & OS & $\begin{array}{l}\text { Windows } 10 \text { Enterprise } \\
\text { 64-bit }\end{array}$ \\
\hline & $\mathrm{CPU}$ & Intel Xeon E-2186G 3.8 GHz (1) & GDB server & Neo4j Desktop 3.5.6 \\
\hline & Memory & 16 GB DDR4 2666 MHz (4) & Python & 3.6 .6 \\
\hline & Hard drive & 1 TB SSD (1) & & \\
\hline & Network & Gigabit Ethernet ports (2) & & \\
\hline \multirow{3}{*}{ Collaborative robots (2) } & Make/Model & $\begin{array}{l}\text { Universal Robots UR3 6-DoF } \\
\text { CB-series [32] }\end{array}$ & Software & 3.12 .0 \\
\hline & Peripheral & $\begin{array}{l}\text { Robotiq 2-Figure 2F-140 } \\
\text { gripper (1) [33] }\end{array}$ & & \\
\hline & Peripheral & $\begin{array}{l}\text { OnRobot 6-axis HEX Force } \\
\text { Torque sensor (1) [34] }\end{array}$ & & \\
\hline \multirow{3}{*}{ Supervisor PLC (1) } & Make/Model & Beckhoff CX2020 [35] & OS & $\begin{array}{l}\text { Windows Embedded } \\
\text { Standard (WES) } 7\end{array}$ \\
\hline & Terminal & $\begin{array}{l}\text { Beckhoff EL6688 external } \\
\text { sync interface (1) [30] }\end{array}$ & PLC IDE & $\begin{array}{l}\text { Beckhoff TwinCAT } \\
\text { 3.1 Build } 4020.28\end{array}$ \\
\hline & Terminal & $\begin{array}{l}\text { Beckhoff EL1808 digital } \\
\text { input (1) }\end{array}$ & TC3 libraries & $\begin{array}{l}\text { TC1200, TF1800, } \\
\text { TF6421 }\end{array}$ \\
\hline \multirow{3}{*}{$\begin{array}{l}\text { CNC emulator } \\
\text { PLCs (4) }\end{array}$} & Make/Model & Beckhoff CX9020 [36] & OS & $\begin{array}{l}\text { Windows Embedded } \\
\text { Compact (CE) } 7\end{array}$ \\
\hline & Terminal & $\begin{array}{l}\text { Beckhoff EL1808 digital } \\
\text { input (1) }\end{array}$ & PLC IDE & $\begin{array}{l}\text { Beckhoff TwinCAT } \\
3.1 \text { Build } 4022.14\end{array}$ \\
\hline & & & TC3 libraries & TC1200 \\
\hline \multirow{5}{*}{$\begin{array}{l}\text { Ethernet-WLAN adapter } \\
\text { /WLAN sniffer ( } 7 \text { ) }\end{array}$} & Make/Model & $\begin{array}{l}\text { Shuttle DL10J NUC } \\
\text { "Intel NUC" [37] }\end{array}$ & OS & $\begin{array}{l}\text { Ubuntu } 14.04 \text { server, } \\
\text { Linux kernel } 4.10 \text {, } \\
\text { 64-bit }\end{array}$ \\
\hline & CPU & $\begin{array}{l}\text { Intel Gemini Lake J4005 } \\
\text { Dual Core }\end{array}$ & $\begin{array}{l}\text { Packet capture\& } \\
\text { measurement }\end{array}$ & TShark, Tcpdump \\
\hline & Memory & 4 GB DDR4 $2400 \mathrm{MHz}(2)$ & Traffic generation & Iperf v2.0.14a \\
\hline & Hard drive & 250 GB SSD (1) & Time sync & linuxptp \\
\hline & Network & $\begin{array}{l}\text { Gigabit Ethernet port (1); } \\
\text { Intel Wireless-AC } 9560 \text { (1) }\end{array}$ & & \\
\hline \multirow{2}{*}{$\begin{array}{l}\text { PTP-capable switches } \\
\text { (2) }\end{array}$} & Make/Model & Cisco IE-4000-8GT4G-E & IOS & $\begin{array}{l}\text { ie4000-universalk9- } \\
\text { mz.152-4.EA5 }\end{array}$ \\
\hline & Network & Gigabit Ethernet ports (12) & & \\
\hline \multirow{2}{*}{ Non-PTP switches (2) } & Make/Model & D-Link 8-port DGS-108 & & \\
\hline & Network & Gigabit Ethernet ports (8) & & \\
\hline Wireless AP (1) & Make/Model & $\begin{array}{l}\text { Netgear AC1900 WLAN router } \\
\text { R7000 (1) }\end{array}$ & Firmware & $\begin{array}{l}\text { DD-WRT } \\
\text { V3.0-R40559 }\end{array}$ \\
\hline TAP devices (7) & Make/Model & $\begin{array}{l}\text { Shark TAP Gigabit network } \\
\text { sniffer }\end{array}$ & & \\
\hline \multirow[t]{2}{*}{ Time server (1) } & Make/Model & $\begin{array}{l}\text { Meinberg M900/GPS PTPv2 } \\
\text { grandmaster [38] }\end{array}$ & Firmware & 6.24 .014 \\
\hline & Network & NTP (1); PTP (1) & & \\
\hline
\end{tabular}




\section{E. Network Diagrams in Testbed Experiments}

IWS TESTBED NETWORK CONNECTIONS (Full Ethernet, No Measurement)

Version: 1.0 .1

Date: 02/13/2020

Prepared by Yongkang Liu

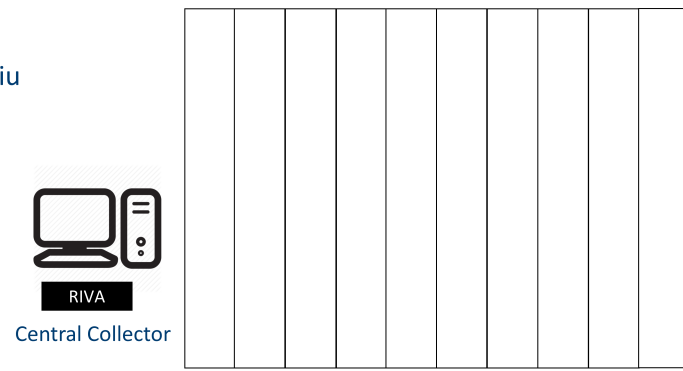

Config. flow + OT RT flow

Trunk port flow

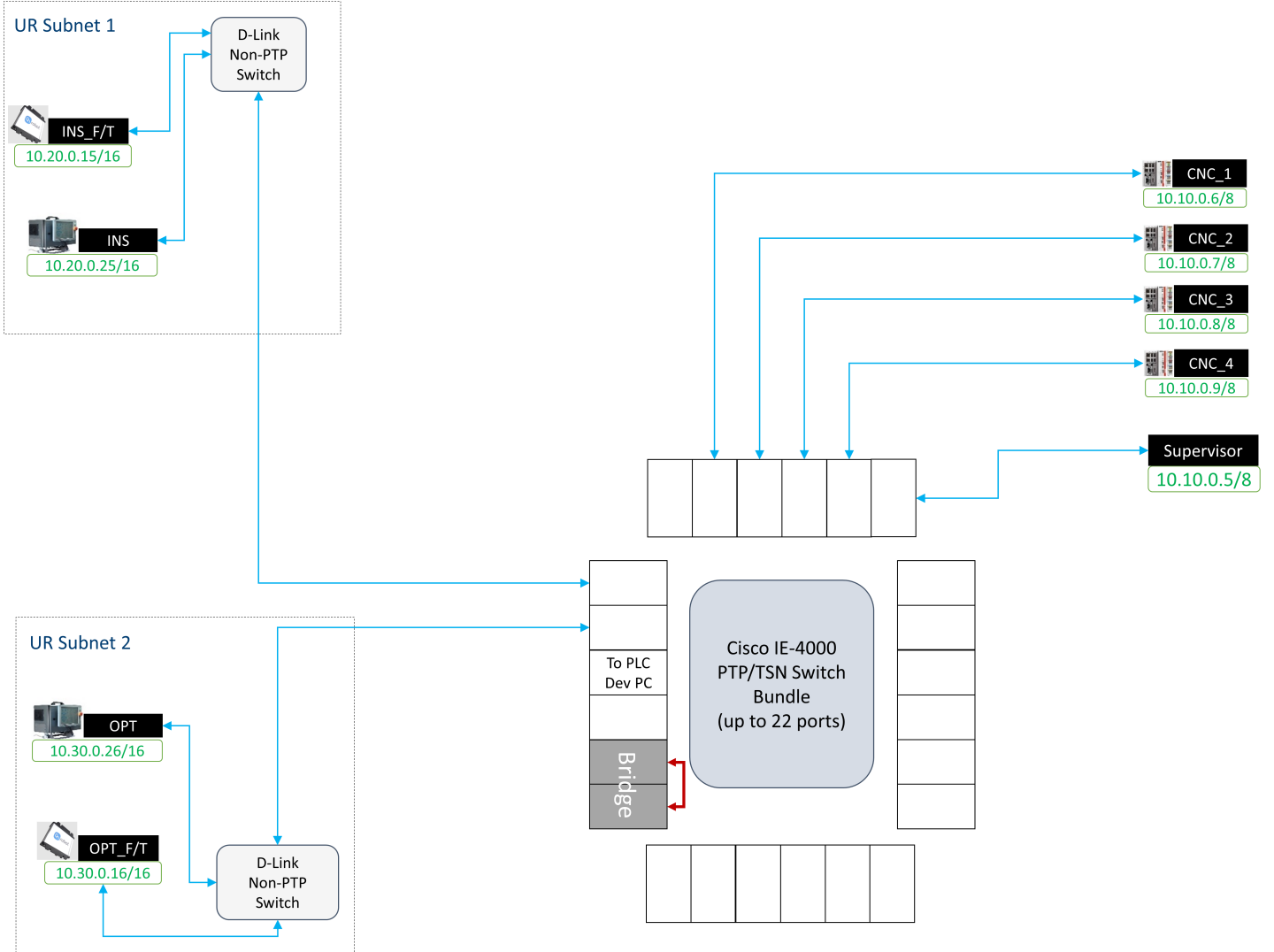

Fig. 63. Testbed network diagram with only work-cell connections 
IWS TESTBED NETWORK CONNECTIONS (Full Ethernet)

Version: 1.0 .1

Date: $02 / 13 / 2020$

Prepared by Yongkang Liu
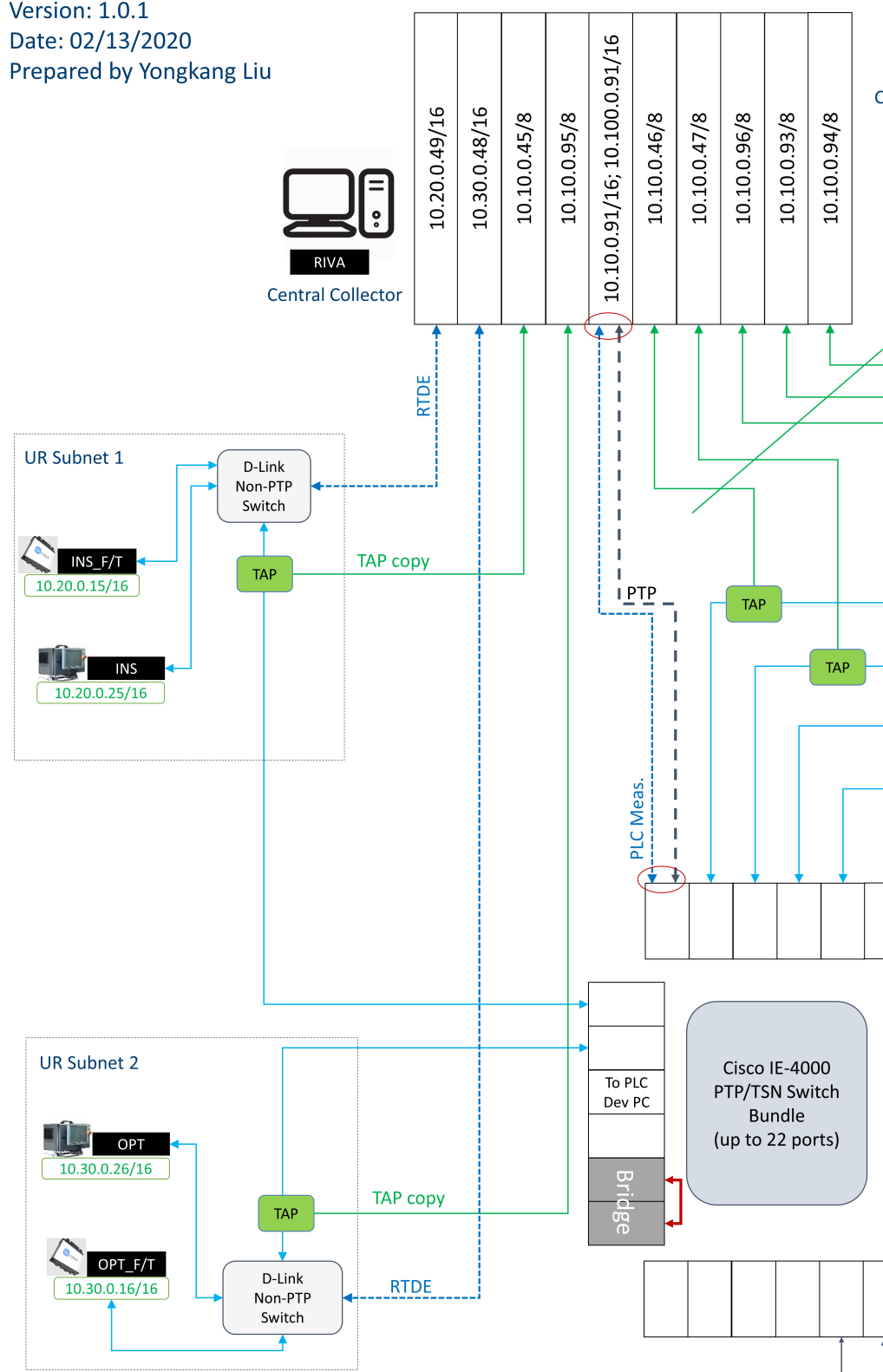
Config. flow only
Config. flow + PTP

$\longleftrightarrow$

Config. flow + OT RT flow

Config. flow + OT meas. data

Network RT flow only

Trunk port flow

Compound flows in a port

(Config.+OT+PTP as shown)

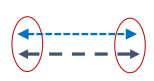

TAP copy
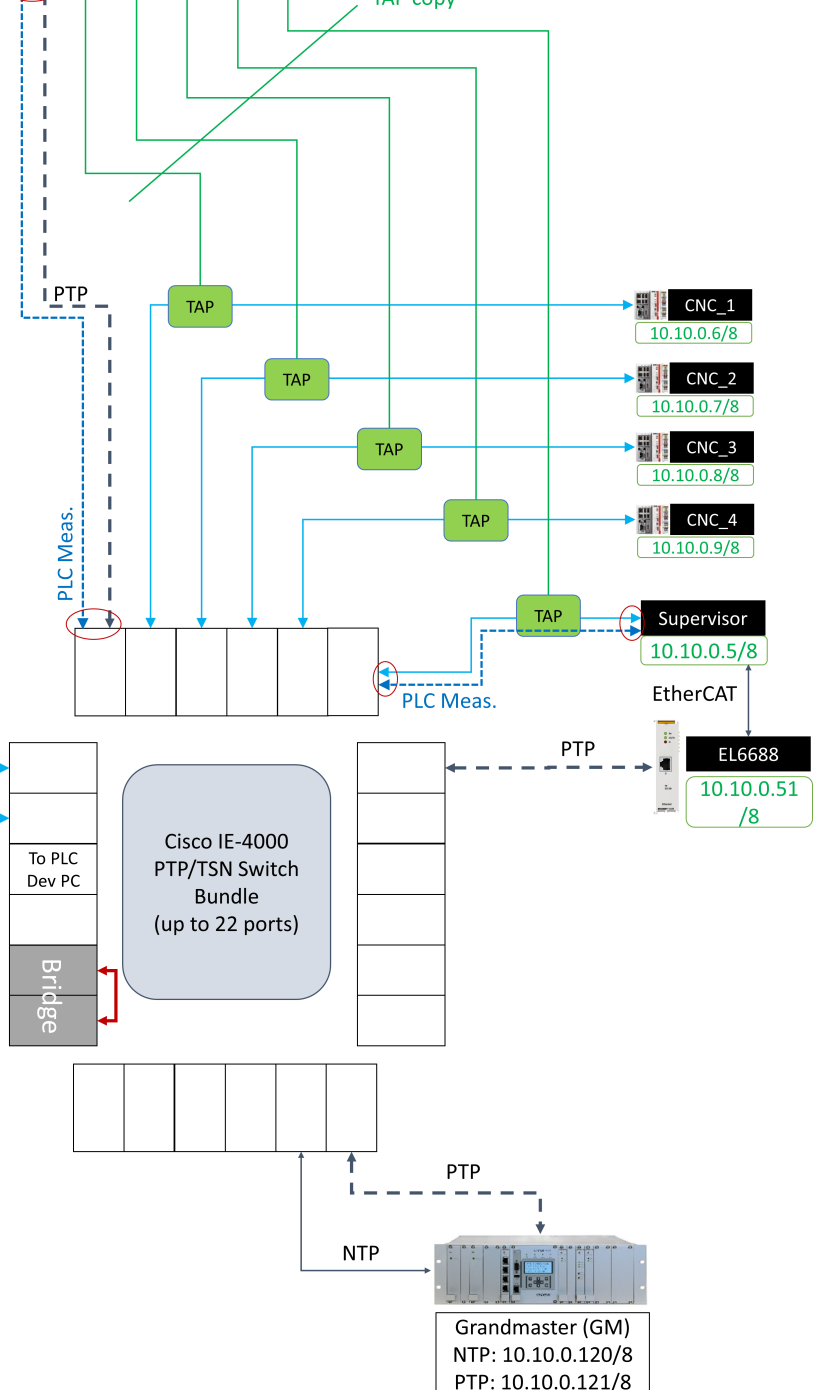

Fig. 64. Testbed network diagram with full wired connections and measurement data links 
IWS TESTBED NETWORK CONNECTIONS (Wireless)

Version: 1.0 .1

Date: $02 / 13 / 2020$

Prepared by Yongkang Liu

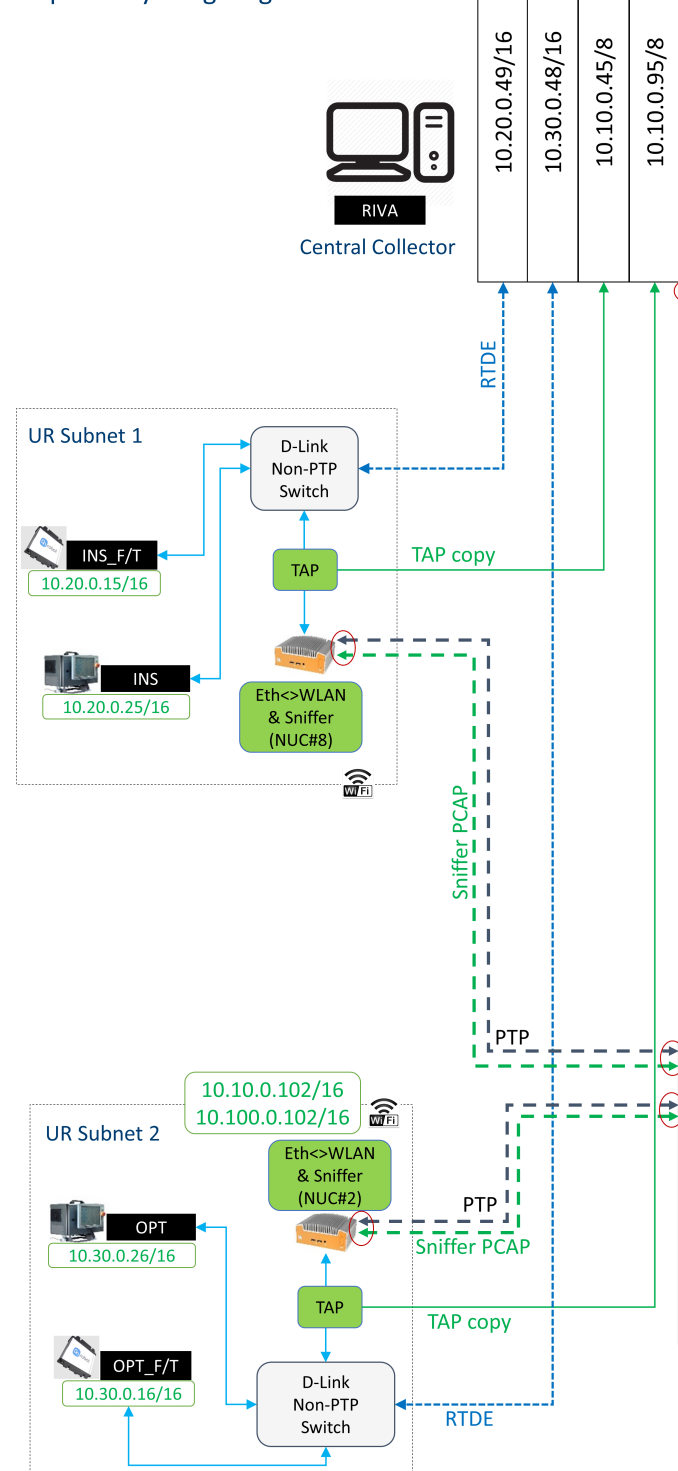

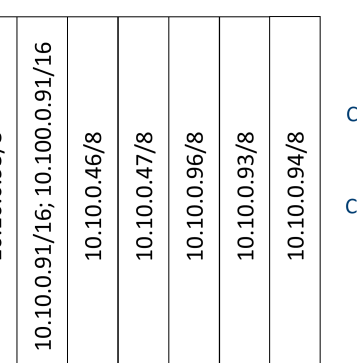

Config. flow only

Config. flow + PTP

Config. flow + OT RT flow

Config. flow + OT meas. data

Network RT flow only

Config. Flow + Ntwk meas. data

Trunk port flow

Compound flows in a port

(Config.+Ntwk+PTP as shown) $\leftarrow---\leftrightarrow$
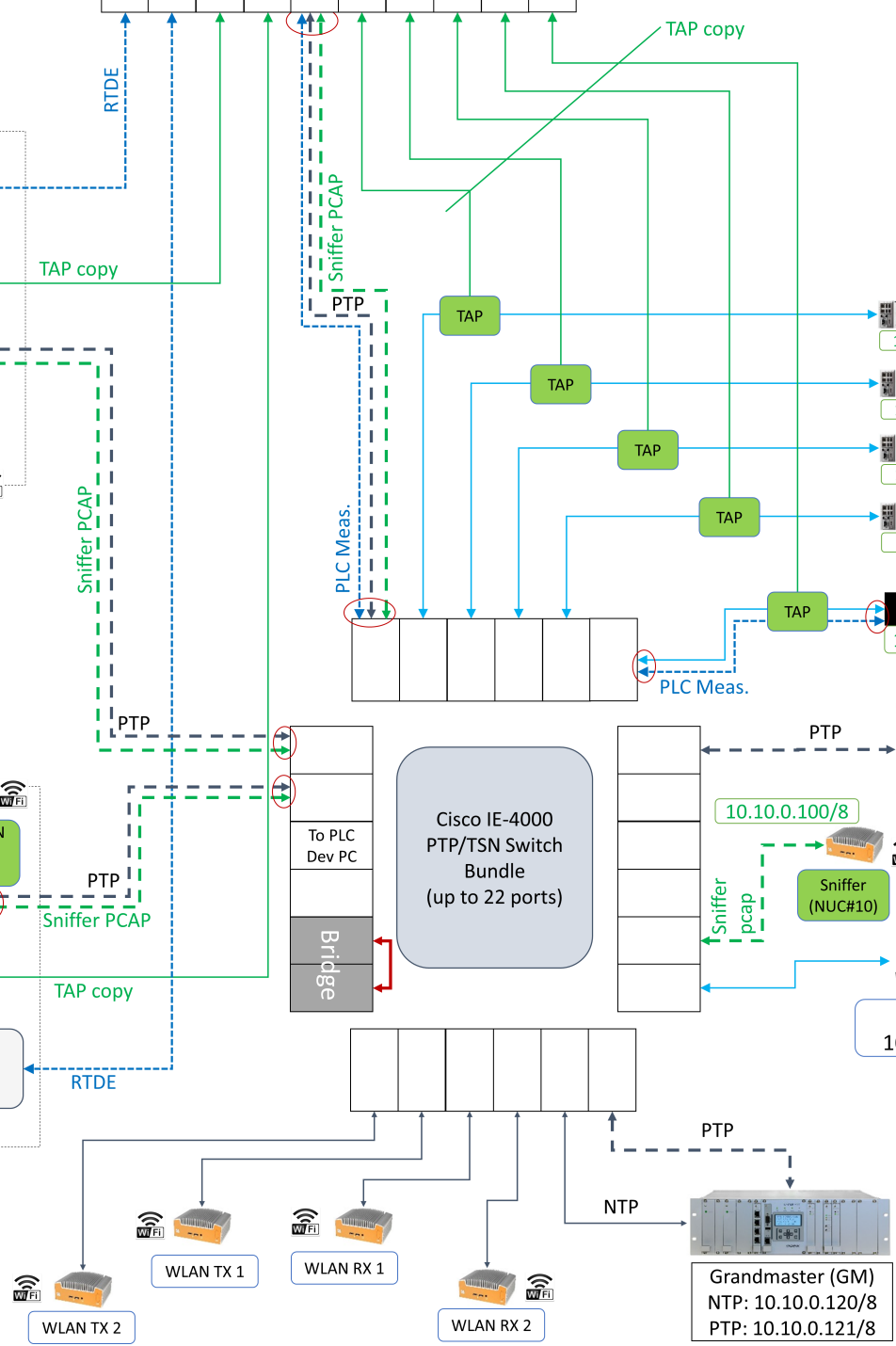

Fig. 65. Testbed network diagram with wireless connections 


\section{F. Acronyms}

5G The fifth-generation

ADS Automation device specification

AGV

Automated guided vehicle

AMS

AP

Automation message specification

ATI

Access point

BC

International atomic time, or "temps atomique international" in French

CLI

Boundary clock

COMM

Command-line interface

CPS

CPU

Communication

Cyber-physical system

CSV

DC

Central processing unit

Comma-separated values

Distributed clock

DDR4

Double data rate 4

DIAG

Diagnostics

DoF

Degree-of-freedom

E2E

End-to-end

EDT

Eastern daylight time

ERP

Enterprise resource planning

EST

Eastern standard time

EtherCAT

Ethernet for control automation technology

F/T

Force torque

FEAT EXTR

GDB

Feature extraction

GDB INSRT

Graph database

GRF TUN

GUI

Database insertion

GVL

Graph tuning

Graphic user interface

HDD

Global variable list

HIL

Hard disk drive

HMI

HW

Hardware in the loop

I/O

Human-machine interface

ID

Hardware

IDE

Input and output

Identification

IEEE

Integrated development environment

Institute of Electrical and Electronics Engineers 
IN

INIT

INS

IOI

IP

ISO

IT

IWS

LAN

MAC

MB

MBAP

MES

NIC

NIST

NoSQL

NTP

NUC

OC

OPT

OS

OT

OUT

PCAP

PDU

PER

PHC

PHY

PLC

POU

pps

PREPROC

PTP

PTPv2

QoS

RDP

RSSI
Input

Initialization

Inspector

I/O module interface

Internet protocol

International Organization for Standardization

Information technology

Industrial wireless systems

Local area network

Medium access control

Megabyte

Modbus application protocol

Manufacturing execution systems

Network interface cards

National Institute of Standards and Technology

Non-relational or non-SQL

Network time protocol

Next unit of computing

Ordinary clock

Operator

Operating system

Operational technology

Output

Packet capture

Protocol data unit

Packet error rate

PTP hardware clock

Physical

Programmable logic controllers

Program organization units

Packets per second

Preprocessing

Precision time protocol

Precision time protocol version 2, also known as IEEE 1588-2008

Quality of service

Remote desktop protocol

Received signal strength indicator 
RTDE Real-time data exchange

RX Reception or receiver

SCHDL Scheduler

SMS Spectrum monitoring service

SNR Signal-to-noise ratio

SQL Structured query language

SSD Solid-state drive

STA State machine

SW Software

TAP Test access point

TB Terabyte

TC Task clock

TCP Transmission control protocol

TDMA Time division multiple access

TPKT ISO transport services on top of the TCP

TSN Time sensitive networking

TX Transmission or transmitter

UE User equipment

UR Universal Robots

URCaps UR robot's extended capabilities, i.e., accessories

UTC Coordinated universal time

WES Windows embedded standard

WIA-FA Wireless networks for industrial automation - factory automation

WLAN Wireless local area network

XML Extensible markup language 